\title{
Categorical Resolutions of Irrational Singularities
}

\author{
Alexander Kuznetsov ${ }^{1,2,3}$ and Valery A. Lunts ${ }^{4}$ \\ ${ }^{1}$ Algebraic Geometry Section, Steklov Mathematical Institute, 8 Gubkin \\ str., Moscow 119991 Russia, ${ }^{2}$ The Poncelet Laboratory, Independent \\ University of Moscow, Moscow, Russia, ${ }^{3}$ Laboratory of Algebraic \\ Geometry, SU-HSE, Moscow, Russia and ${ }^{4}$ Department of Mathematics, \\ Indiana University, Bloomington, IN 47405, USA
}

Correspondence to be sent to: akuznet@mi.ras.ru

We show that the derived category of any singularity over a field of characteristic 0 can be embedded fully and faithfully into a smooth triangulated category that has a semiorthogonal decomposition with components equivalent to derived categories of smooth varieties. This provides a categorical resolution of the singularity.

\section{Introduction}

Resolution of singularities is one of central concepts in algebraic geometry. In many cases, it allows one to reduce the complicated geometry of singular schemes to a much more tractable geometry of smooth schemes. From the categorical point of view a resolution $\pi: X \rightarrow Y$ manifests itself in a pair of adjoint functors

$$
L \pi^{*}: \mathbf{D}(Y) \rightarrow \mathbf{D}(X) \quad \text { and } \quad R \pi_{*}: \mathbf{D}(X) \rightarrow \mathbf{D}(Y)
$$

(the derived pullback and the derived pushforward) between the derived categories of quasicoherent sheaves on $X$ and $Y$, respectively. The functors are related by the 
projection formula

$$
R \pi_{*}\left(L \pi^{*}(F)\right) \cong F \stackrel{\mathbb{L}}{\otimes} R \pi_{*} \mathcal{O}_{X},
$$

which shows that, from the categorical point of view, there are two completely different situations:

(1) the canonical morphism $\mathcal{O}_{Y} \rightarrow R \pi_{*} \mathcal{O}_{X}$ is an isomorphism;

(2) the canonical morphism $\mathcal{O}_{Y} \rightarrow R \pi_{*} \mathcal{O}_{X}$ is not an isomorphism.

If (1) holds, one says that $Y$ has rational singularities. In this case $R \pi_{*} \circ L \pi^{*} \cong \mathrm{id}$, hence the pullback functor $L \pi^{*}$ is fully faithful, so the singular category $\mathbf{D}(Y)$ embeds into a smooth category $\mathbf{D}(X)$. This embedding allows the reductions of geometrical questions on $Y$ to those on $X$ that we mentioned above. On a contrary, if (2) holds, the functor $L \pi^{*}$ is not fully faithful, so it does not provide such a reduction. So, from a categorical point of view, the usual resolution of a scheme that has irrational singularities is not a resolution at all!

The goal of the present paper is to show that, even for a scheme $Y$ with irrational singularities, one can construct a categorical resolution by gluing appropriately several derived categories of smooth varieties. To be more precise, for each separated scheme of finite type $Y$ over a field $\mathbb{k}$ of characteristic 0 , we construct a nice triangulated category $\mathscr{T}$ with an adjoint pair of triangulated functors $\pi^{*}: \mathbf{D}(Y) \rightarrow \mathscr{T}$ and $\pi_{*}: \mathscr{T} \rightarrow \mathbf{D}(Y)$ such that $\pi_{*} \circ \pi^{*} \cong$ id so that $\pi^{*}$ is fully faithful; moreover, $\mathscr{T}$ enjoys a number of useful properties; see Definition 1.3.

Before going to precise definitions we have to explain what we mean by saying that $\mathscr{T}$ is nice. First of all, the category $\mathscr{T}$ has to be smooth. The notion of smoothness is formulated in terms of DG-categories (an introduction into the subject can be found in Section 3), so we have to start with recalling what is a smooth DG-category.

Definition 1.1. A small DG-category $\mathscr{D}$ is smooth if the diagonal bimodule $\mathscr{D}$ is perfect. In other words, if it is contained in the smallest Karoubian-closed triangulated subcategory of the derived category $\mathbf{D}\left(\mathscr{D}^{\mathrm{op}} \otimes \mathscr{D}\right)$ generated by representable bimodules.

This leads to the following definitions.

Definition 1.2. A cocomplete, compactly generated, triangulated category $\mathscr{T}$ is smooth if there exists a smooth DG-category $\mathscr{D}$ such that its derived category $\mathbf{D}(\mathscr{D})$ is equivalent to $\mathscr{T}$ as a triangulated category. 
It is well known (see [26]) that if $X$ is a smooth variety, then the derived category $\mathbf{D}(X)$ is smooth.

Now, let us give a precise definition of a categorical resolution. Recall that an object $F$ of a triangulated category $\mathscr{T}$ is compact if the functor $\operatorname{Hom}_{\mathscr{T}}(F,-)$ commutes with arbitrary direct sums. The subcategory $\mathscr{T}^{\mathrm{c}} \subset \mathscr{T}$ of compact objects in $\mathscr{T}$ is triangulated. Compact objects in $\mathbf{D}(Y)$, the unbounded derived category of quasicoherent sheaves on a separated scheme of finite type $Y$, are perfect complexes, that is, the objects that are locally quasiisomorphic to finite complexes of locally free sheaves of finite rank. The perfect complexes form a triangulated subcategory of $\mathbf{D}^{b}(\operatorname{coh}(Y))$ which we denote by $\mathrm{D}^{\text {perf }}(Y)$.

Definition 1.3 ([15]). A categorical resolution of a scheme $Y$ is a smooth, cocomplete, compactly generated, triangulated category $\mathscr{T}$ with an adjoint pair of triangulated functors

$$
\pi^{*}: \mathbf{D}(Y) \rightarrow \mathscr{T} \text { and } \pi_{*}: \mathscr{T} \rightarrow \mathbf{D}(Y)
$$

such that

(1) $\pi_{*} \circ \pi^{*}=\mathrm{id}$;

(2) both $\pi^{*}$ and $\pi_{*}$ commute with arbitrary direct sums;

(3) $\pi_{*}\left(\mathscr{T}^{\mathrm{c}}\right) \subset \mathbf{D}^{b}(\operatorname{coh}(Y))$.

Note that the second property implies that $\pi^{*}\left(\mathbf{D}^{\text {perf }}(Y)\right) \subset \mathscr{T}^{\mathrm{c}}$ (see Lemma 2.10).

If $\pi: X \rightarrow Y$ is a usual resolution and $Y$ has rational singularities, then the category $\mathscr{T}=\mathbf{D}(X)$ with the functors $L \pi^{*}$ and $R \pi_{*}$ is a categorical resolution of $Y$. Note, however, that if the singularities of $Y$ are not rational, then $\mathbf{D}(X)$ is not a categorical resolution of $Y$ since the composition $R \pi_{*} \circ L \pi^{*}$ is isomorphic to the tensor product with $R \pi_{*} \mathcal{O}_{X}$, which is not the identity functor.

To formulate the main result of the paper, we need one more notion. As we already have said, the resolution that we construct is a nice triangulated category. In fact, it is even nicer than just a smooth triangulated category. It has a very geometric nature-to be more precise, it has a semiorthogonal decomposition with all components being derived categories of smooth algebraic varieties. We call such categories strongly geometric. Recall also that a cocomplete, compactly generated, triangulated category $\mathscr{T}$ is proper if the category $\mathscr{T}^{\mathrm{c}}$ is Ext-finite, which means that the vector space $\oplus_{i \in \mathbb{Z}} \operatorname{Hom}(F, G[i])$ is finite-dimensional for all $F, G \in \mathscr{T}^{\mathrm{c}}$.

The main result of the paper is the following theorem. 
Theorem 1.4. Any separated scheme of finite type $Y$ over a field of characteristic 0 has a categorical resolution by a strongly geometric triangulated category $\mathscr{T}$. If $Y$ is proper, then so is the resolving category $\mathscr{T}$.

Note that we do not put any restrictions on the singularity of $Y$. In fact, it may be not normal, reducible, and even nonreduced; the construction still works!

As we shall soon explain, the varieties whose derived categories appear as components of the categorical resolution that we construct are strongly related to the usual process of desingularization of the reduced scheme $Y_{\text {red }}$-if we fix a usual resolution $X \rightarrow Y_{\text {red }}$ by a sequence of blowups with smooth centers $Z_{0}, Z_{1}, \ldots, Z_{m-1}$, then the components of $\mathscr{T}$ are $\mathbf{D}\left(Z_{0}\right), \mathbf{D}\left(Z_{1}\right), \ldots, \mathbf{D}\left(Z_{m-1}\right)$ (each of them may be repeated several times!) and $\mathbf{D}(X)$ (with repetitions if $Y$ itself is not reduced).

Let us outline the construction that we use.

First of all, we use heavily the machinery of DG-categories (see Section 3 for an introduction into the subject). Accordingly, we use a DG-version of the definition of a categorical resolution.

Definition 1.5. A partial categorical DG-resolution of a small pretriangulated DG-category $\mathscr{D}$ is a small pretriangulated DG-category $\tilde{\mathscr{D}}$ with a DG-functor $\pi: \mathscr{D} \rightarrow \tilde{\mathscr{D}}$, which induces a fully faithful functor on homotopy categories. If additionally $\tilde{\mathscr{D}}$ is smooth, we say that it is a categorical DG-resolution.

The first instrument of the construction is the notion of a gluing of DG-categories. Given two small DG-categories $\mathscr{D}_{1}, \mathscr{D}_{2}$ and a $\mathscr{D}_{1}-\mathscr{D}_{2}$-bimodule $\varphi$, we define a DG-category $\mathscr{D}_{1} \times{ }_{\varphi} \mathscr{D}_{2}$. The definition can be found in Section 4; here we will just say that it is a straightforward generalization of an upper triangular algebra, with the diagonal entries being two given algebras and the upper diagonal entry being a bimodule over these algebras. We show that the gluing of two pretriangulated categories is itself pretriangulated. The derived and homotopy categories of the gluing have semiorthogonal decompositions

$$
\left[\mathscr{D}_{1} \times_{\varphi} \mathscr{D}_{2}\right]=\left\langle\left[\mathscr{D}_{1}\right],\left[\mathscr{D}_{2}\right]\right\rangle, \quad \mathbf{D}\left(\mathscr{D}_{1} \times_{\varphi} \mathscr{D}_{2}\right)=\left\langle\mathbf{D}\left(\mathscr{D}_{1}\right), \mathbf{D}\left(\mathscr{D}_{2}\right)\right\rangle
$$

The quasiequivalence class of the gluing depends only on the quasiisomorphism class of the gluing bimodule, and the gluing is smooth if and only if both components $\mathscr{D}_{1}$ and $\mathscr{D}_{2}$ are smooth and the gluing bimodule is perfect (see Proposition 4.9 and [20]).

Another instrument is the category of $\mathscr{A}$-modules. Given a scheme $S$, an integer $n$, and an ideal $\mathfrak{r} \subset \mathcal{O}_{S}$ such that $\mathfrak{r}^{n}=0$, we define the sheaf of $\mathcal{O}_{S}$-algebras $\mathscr{A}=\mathscr{A}_{S, \mathfrak{r}, n}$ as a 
certain subalgebra in $\operatorname{End}_{\mathcal{O}_{S}}\left(\mathcal{O}_{S} \oplus \mathcal{O}_{S} / \mathfrak{r}^{n-1} \oplus \cdots \oplus \mathcal{O}_{S} / \mathfrak{r}\right)$; see (30) for the precise formula. The ringed space $\left(S, \mathscr{A}_{S, \mathfrak{r}, n}\right)$ is called an $\mathscr{A}$-space. One of the points of the paper is that $\mathscr{A}$-spaces share many good properties with schemes (and have some advantages), so one can use them as building blocks for constructing triangulated categories of geometric interest.

The category $\operatorname{Qcoh}(\mathscr{A})$ of quasicoherent $\mathscr{A}$-modules on $S$ is an abelian category and we prove that its derived category $\mathbf{D}(\mathscr{A})$ gives a categorical resolution of $\mathbf{D}(S)$ if the closed subscheme $S_{0} \subset S$ corresponding to the ideal $\mathfrak{r}$ is smooth. Moreover, $\mathbf{D}(\mathscr{A})$ comes with semiorthogonal decompositions

$$
\begin{aligned}
\mathbf{D}(\mathscr{A}) & =\left\langle\mathbf{D}\left(S_{0}\right), \mathbf{D}\left(S_{0}\right), \ldots, \mathbf{D}\left(S_{0}\right)\right\rangle \\
\mathbf{D}^{b}(\operatorname{coh}(\mathscr{A})) & =\left\langle\mathbf{D}^{b}\left(\operatorname{coh}\left(S_{0}\right)\right), \mathbf{D}^{b}\left(\operatorname{coh}\left(S_{0}\right)\right), \ldots, \mathbf{D}^{b}\left(\operatorname{coh}\left(S_{0}\right)\right)\right\rangle
\end{aligned}
$$

(the number of components equals $n$ ). Moreover, taking appropriate DG-enhancement $\mathscr{D}(\mathscr{A})$ of the derived category of perfect $\mathscr{A}$-modules gives a categorical DG-resolution of $\mathscr{D}(S)$, a DG-model of the category of perfect complexes on $S$.

Now the main construction looks as follows. We consider a blowup $f: Y_{1} \rightarrow Y$ with a smooth center $Z \subset Y$. Then, we show that, for $n$ sufficiently, large the $n$th infinitesimal neighborhood $S$ of the subscheme $Z$ (i.e., the subscheme with $I_{S}=I_{Z}^{n}$ ) has the following important property:

$$
R f_{*} I_{f^{-1}(S)} \cong I_{S}
$$

Here, $f^{-1}(S)$ is the scheme-theoretic preimage of $S$. We call such a subscheme $S$ a nonrational locus for $f$. We consider the nilpotent ideal $\mathfrak{r}:=I_{Z} / I_{S}$ on $S$, so that we have $S_{0}=Z$, and the category $\operatorname{Qcoh}\left(\mathscr{A}_{S}\right)=\operatorname{Qcoh}\left(\mathscr{A}_{S, \mathfrak{r}, n}\right)$ of $\mathscr{A}_{S}$-modules. Furthermore, we construct a $\mathscr{D}\left(\mathscr{A}_{S}\right)$ - $\mathscr{D}\left(Y_{1}\right)$-bimodule $\varphi$ such that the gluing $\mathscr{D}\left(\mathscr{A}_{S}\right) \times_{\varphi} \mathscr{D}\left(Y_{1}\right)$ is a partial categorical resolution of $\mathscr{D}(Y)$. Of course, this is not yet a resolution-there is no reason for $Y_{1}$ to be smooth. However, this serves as a step of induction. With a wise choice of the first blowup center $Z$ the singularities of $Y_{1}$ are more simple than those of $Y$ and we can assume by induction that $\mathscr{D}\left(Y_{1}\right)$ has a categorical DG-resolution $\mathscr{D}_{1}$. Then, we replace the gluing $\mathscr{D}\left(\mathscr{A}_{S}\right) \times_{\varphi} \mathscr{D}\left(Y_{1}\right)$ by $\mathscr{D}:=\mathscr{D}\left(\mathscr{A}_{S}\right) \times_{\tilde{\varphi}} \mathscr{D}_{1}$, where $\tilde{\varphi}=\varphi \stackrel{\mathbb{L}}{\otimes} \mathscr{D}\left(Y_{1}\right) \mathscr{D}_{1}$ (this procedure is called regluing) and show that $\mathscr{D}$ is a categorical DG-resolution of $\mathscr{D}(Y)$.

The base of the induction is the case of a (possibly nonreduced) scheme $Y$ such that the associated reduced scheme $Y_{\text {red }}$ is smooth. In this case, the resolution is again provided by the derived category of $\mathscr{A}$-modules $\mathbf{D}\left(\mathscr{A}_{Y}\right)$. Thus, we use the category of 
$\mathscr{A}$-modules both in the base and in the step of the induction. Note also that this category is essential for the construction even if the original scheme $Y$ is reduced since the nonrational locus typically has nonreduced structure which has to be resolved.

Note that each step of the above construction seriously depends on a choice of integer $n$ (which, as mentioned above, can be chosen arbitrarily as soon as it is sufficiently large). It is worth mentioning that increasing $n$ by 1 has an effect of adding one more semiorthogonal component equivalent to $\mathbf{D}(Z)$ to the resolving category. This is analogous to making an extra smooth blowup of a resolution of singularities in geometric situation. Thus, if one would like to construct "smaller" resolution, one should choose $n$ as small as possible.

The constructed resolution has several important properties. First of all, replacing $Y$ by its open subsets, one obtains a presheaf of DG-categories on $Y$. If $Y$ is generically reduced, then, on a sufficiently small open subset $U \subset Y$, the corresponding DGcategory coincides with $\mathscr{D}(U)$ - this expresses birationality of the resolution. If $Y$ comes with an action of a group $G$, one can choose a resolution on which the same group $G$ acts in a compatible way.

Since our categorical resolution requires a usual resolution as an input it is restricted to characteristic 0 . On the other hand, in cases where a usual resolution is known in positive characteristic (e.g., in dimension 3 and sufficiently large characteristic), our construction can be applied to give a categorical resolution.

We expect that both the result and the approach of the paper will be very useful for investigation of derived categories of singular varieties, especially those with irrational singularities. For example, one can use our results to investigate generalized Jacobians of singular curves (including nonplanar curves).

Another application that we originally had in mind was to the following conjecture suggested by M. Kontsevich.

Conjecture 1.6. Let $Y$ be a separated scheme of finite type over a field of characteristic zero. Then, the bounded derived category of coherent sheaves $\mathbf{D}^{b}(\operatorname{coh}(Y))$ is homotopically finitely presented.

We refer the reader to [26] for the definition of homotopically finitely presented (hfp for short) DG-categories. Here, we just mention that one of the ways to prove that a category is hfp is by showing that it is a quotient of another hfp category by subcategory generated by one object. So, we suggested to use the categorical resolution constructed here (it is hfp because it is strongly geometric) as the category to start with. 
This approach was accomplished by Efimov [11]. In Efimov's proof of Kontsevich's Conjecture, it is essential that one can increase the width of the blowup centers (integer $n$ mentioned above); so even for varieties with rational singularities (when the usual resolution is categorical) our approach gives new significant results!

Finally, let us explain the history of notions of a categorical resolution in the literature. To our best knowledge, the first example of a categorical resolution was a theorem of Auslander [2]. It appeared in the language of representations of Artin algebras. This theorem served as a guideline for us and is a prototype of our $\mathcal{A}$-space.

Categorical resolutions with the accent on triangulated categories (rather than abelian ones) seem to have first appeared in [16]. The examples given in that paper were intended to show that a categorical resolution may be better than the usual one in some aspects.

The first definition of a categorical resolution is due to Bondal and Orlov [9]. The definition suggested in [9] is much stronger than one used in our paper-it is assumed there that the resolving category is the bounded derived category of an abelian category of finite homological dimension (in particular, the resolving category has a bounded tstructure), and moreover that $\mathbf{D}^{b}(\operatorname{coh}(Y))$ is a localization of that category. We believe that the first assumption is irrelevant and too restrictive; this is why we construct resolutions as derived categories of DG-categories (note, however, that the resolution $\mathbf{D}\left(\mathscr{A}_{S}\right)$ of a nonreduced scheme $S$ with smooth $S_{0}$ enjoys all the properties asked for in [9]). On the other hand, we believe that the resolutions that we construct enjoy the second property-that $\mathbf{D}^{b}(\operatorname{coh}(Y))$ is the quotient of $\mathscr{T}^{c}$. As it follows from results of Efimov [11], this is true if the integers $n$ in the construction are large enough. In fact, we believe that this is true for any choice of these integers.

Another notion, a noncommutative crepant resolution, was introduced by Van den Bergh [28]. It was even more restrictive than the original definition of Bondal and Orlov. In addition, it was assumed that the resolving abelian category can be realized as the category of sheaves of modules over a certain sheaf of algebras on $Y$ with nice homological properties.

On the other hand, in [15] the definition of a categorical resolution (in the context of small categories) used here first appeared. However, it was expected that such resolution may exist only if $Y$ has rational singularities, and the point of the paper was in finding minimal resolutions by starting from a commutative resolution and then shrinking it by chopping out some irrelevant semiorthogonal components. So, in a sense the result of the present paper shows that the same approach may be used for nonrational singularities as well. 
Finally, in papers $[17,18]$ the notion of a categorical resolution in the context of big categories was considered. In particular, it was shown in [18] that one can construct a categorical resolution of $\mathbf{D}(Y)$ by a so-called smooth poset scheme if and only if $Y$ has Du Bois singularities. A smooth poset scheme is obtained by "gluing" a finite number of smooth schemes $X_{\alpha}$ (indexed by a poset) along arbitrary morphisms $f_{\alpha \beta}: X_{\alpha} \rightarrow X_{\beta}$ (if $\alpha \geq \beta$ ). Thus, in case $Y$ has Du Bois singularities, its categorical resolution (as in Theorem 1.4) can be chosen in a more geometric way: one only needs to glue smooth schemes along honest morphisms, and $\mathscr{A}$-modules can be avoided.

\section{Preliminaries on Triangulated Categories}

General reference for the material on triangulated categories is $[6,8,9]$.

\subsection{Semiorthogonal decompositions}

Let $\mathbb{k}$ be a field and $\mathscr{T}$ be a $\mathbb{k}$-linear triangulated category.

Definition 2.1 ([6, 8]). A semiorthogonal decomposition of triangulated category $\mathscr{T}$ is a collection $\mathscr{T}_{1}, \ldots, \mathscr{T}_{m}$ of strictly full triangulated subcategories in $\mathscr{T}$ (components of the decomposition) such that

(1) $\operatorname{Hom}_{\mathscr{T}}\left(\mathscr{T}_{i}, \mathscr{T}_{j}\right)=0$ for $i>j$;

(2) for any $F \in \mathscr{T}$ there is a chain of maps

$$
0=F_{m} \rightarrow F_{m-1} \rightarrow \cdots \rightarrow F_{1} \rightarrow F_{0}=F
$$

such that Cone $\left(F_{i} \rightarrow F_{i-1}\right) \in \mathscr{T}_{i}$ for all $i=1, \ldots, m$.

If only the first property holds, we will say that $\mathscr{T}_{1}, \ldots, \mathscr{T}_{m}$ is a semiorthogonal collection of subcategories. We will use notation

$$
\mathscr{T}=\left\langle\mathscr{T}_{1}, \ldots, \mathscr{T}_{m}\right\rangle
$$

to express a semiorthogonal decomposition of $\mathscr{T}$ with components $\mathscr{T}_{1}, \ldots, \mathscr{T}_{m}$.

It follows from the definition that the chain of maps (1) is functorial in $F$, and moreover, the cones of the maps in the chain are also functorial. In other words, there 
are functors

$$
\mathscr{T} \rightarrow \mathscr{T}_{i}, \quad F \mapsto \mathbf{p}_{i}(F):=\operatorname{Cone}\left(F_{i} \rightarrow F_{i-1}\right)
$$

known as the projection functors of the semiorthogonal decomposition.

For future convenience, we will need to restate the definition of a semiorthogonal decomposition in the special case of $m=2$ components. In this case, the chain (1) for $F \in \mathscr{T}$ looks as $0 \rightarrow F^{\prime} \rightarrow F$ and the conditions are that $F^{\prime}=\operatorname{Cone}\left(0 \rightarrow F^{\prime}\right) \in \mathscr{T}_{2}$ and Cone $\left(F^{\prime} \rightarrow F\right) \in \mathscr{T}_{1}$. In other words, $\mathscr{T}=\left\langle\mathscr{T}_{1}, \mathscr{T}_{2}\right\rangle$ is a semiorthogonal decomposition if and only if

(1) $\operatorname{Hom}\left(\mathscr{T}_{2}, \mathscr{T}_{1}\right)=0$ and

(2) for each $F \in \mathscr{T}$ there is a distinguished triangle

$$
\mathbf{p}_{2}(F) \rightarrow F \rightarrow \mathbf{p}_{1}(F) \rightarrow \mathbf{p}_{2}(F)[1]
$$

with $\mathbf{p}_{i}(F) \in \mathscr{T}_{i}$.

One can also express the last property by saying that each object in $\mathscr{T}$ can be represented as a cone of a morphism from an object of $\mathscr{T}_{1}$ to an object of $\mathscr{T}_{2}$.

The following result is well known.

Lemma 2.2 ([6]). Assume that $\mathscr{T}_{1}, \mathscr{T}_{2} \subset \mathscr{T}$ is a semiorthogonal pair of full triangulated subcategories, such that

(1) the embedding functor $i_{1}: \mathscr{T}_{1} \rightarrow \mathscr{T}$ has a left adjoint $i_{1}^{*}: \mathscr{T} \rightarrow \mathscr{T}_{1}$ and

(2) the embedding functor $i_{2}: \mathscr{T}_{2} \rightarrow \mathscr{T}$ has a right adjoint $i_{2}^{!}: \mathscr{T} \rightarrow \mathscr{T}_{2}$.

Then, there is a semiorthogonal decomposition

$$
\mathscr{T}=\left\langle\mathscr{T}_{1},{ }^{\perp} \mathscr{T}_{1} \cap \mathscr{T}_{2}^{\perp}, \mathscr{T}_{2}\right\rangle
$$

where

$$
\begin{gathered}
\perp \mathscr{T}_{1}=\left\{T \in \mathscr{T} \mid \operatorname{Hom}\left(T, \mathscr{T}_{1}\right)=0\right\}=\operatorname{Ker} i_{1}^{*}, \\
\mathscr{T}_{2}^{\perp}=\left\{T \in \mathscr{T} \mid \operatorname{Hom}\left(\mathscr{T}_{2}, T\right)=0\right\}=\operatorname{Ker} i_{2}^{!} .
\end{gathered}
$$

In particular, if $\perp^{\perp} \mathscr{T}_{1} \cap \mathscr{T}_{2}^{\perp}=0$, then $\mathscr{T}=\left\langle\mathscr{T}_{1}, \mathscr{T}_{2}\right\rangle$. Moreover, $\mathbf{p}_{1}=i_{1} i_{1}^{*}, \mathbf{p}_{2}=i_{2} i_{2}$. 


\subsection{The gluing functor}

Let $\mathscr{T}=\left\langle\mathscr{T}_{1}, \mathscr{T}_{2}\right\rangle$ be a semiorthogonal decomposition .

Definition 2.3. The gluing bifunctor of a semiorthogonal decomposition $\mathscr{T}=\left\langle\mathscr{T}_{1}, \mathscr{T}_{2}\right\rangle$ is the functor $\Phi: \mathscr{T}_{1}^{\mathrm{op}} \times \mathscr{T}_{2} \rightarrow \mathbb{k}$-mod defined by

$$
\Phi\left(F_{1}, F_{2}\right)=\operatorname{Hom}_{\mathscr{T}}\left(F_{1}, F_{2}[1]\right) .
$$

For each object $F_{2} \in \mathscr{T}_{2}$, we have a contravariant cohomological functor $\Phi\left(-, F_{2}\right)$ : $\mathscr{T}_{1}^{\text {op }} \rightarrow \mathbb{k}$-mod. Assume that, for all $F_{2} \in \mathscr{T}_{2}$, this functor is representable. Then, there exists a functor $\phi: \mathscr{T}_{2} \rightarrow \mathscr{T}_{1}$ with a functorial isomorphism

$$
\Phi\left(F_{1}, F_{2}\right) \cong \operatorname{Hom}_{\mathscr{T}_{1}}\left(F_{1}, \phi\left(F_{2}\right)\right) .
$$

If such a functor $\phi$ exists, then it is unique up to an isomorphism.

Definition 2.4. The functor $\phi$ with the property (2) is called the gluing functor of a semiorthogonal decomposition $\mathscr{T}=\left\langle\mathscr{T}_{1}, \mathscr{T}_{2}\right\rangle$.

It is easy to see that if $i_{1}$ has also right adjoint $i_{1}^{!}$, then

$$
\phi=i_{1}^{!} i_{2}[1]
$$

is the gluing functor.

The importance of the gluing functor is shown by the following lemma.

Lemma 2.5. Assume $\mathscr{T}=\left\langle\mathscr{T}_{1}, \mathscr{T}_{2}\right\rangle$ and a gluing functor $\phi$ exists. To give an object $F$ of $\mathscr{T}$ is equivalent to giving an object $F_{1}$ of $\mathscr{T}_{1}$, an object $F_{2}$ of $\mathscr{T}_{2}$, and a morphism $f: F_{1} \rightarrow \phi\left(F_{2}\right)$ in $\mathscr{T}_{1}$.

Proof. Let $F \in \mathscr{T}$ and put $F_{i}=\mathbf{p}_{i}(F)$. Then, we have a distinguished triangle

$$
F_{2} \rightarrow F \rightarrow F_{1} \rightarrow F_{2}[1]
$$

The connecting morphism $F_{1} \rightarrow F_{2}[1]$ is given by an element $f$ of $\operatorname{Hom}_{\mathscr{T}}\left(F_{1}, F_{2}[1]\right)$ that is of $\operatorname{Hom}_{\mathscr{T}_{1}}\left(F_{1}, \phi\left(F_{2}\right)\right)$. Thus, we produce $F_{1}, F_{2}$, and $f$ from $F$. Conversely, if $F_{1}, F_{2}$, and $f$ are given, we interpret $f$ as a morphism $F_{1} \rightarrow F_{2}[1]$ in $\mathscr{T}$ and take $F=\operatorname{Cone}\left(F_{1} \rightarrow\right.$ $\left.F_{2}[1]\right)[-1]$. 
This lemma motivates the definition of the gluing category in Section 4.

\subsection{Compact objects}

Recall that a triangulated category $\mathscr{T}$ is cocomplete if it has arbitrary small direct sums.

Definition 2.6 ([21]). An object $F \in \mathscr{T}$ in a cocomplete triangulated category $\mathscr{T}$ is compact if, for any set of objects $G_{i} \in \mathscr{T}$, the canonical morphism

$$
\oplus \operatorname{Hom}\left(F, G_{i}\right) \rightarrow \operatorname{Hom}\left(F, \oplus G_{i}\right)
$$

is an isomorphism.

Compact objects of a cocomplete triangulated category $\mathscr{T}$ form a triangulated subcategory in $\mathscr{T}$ which we denote by $\mathscr{T}^{\mathrm{c}}$.

Definition 2.7 ([21]). Triangulated category $\mathscr{T}$ is generated by a class $\mathcal{S}$ of compact objects if $\mathcal{S}^{\perp}=0$. In particular, $\mathscr{T}$ is compactly generated if $\left(\mathscr{T}^{\mathrm{c}}\right)^{\perp}=0$.

An important result of Neeman which we will frequently use is the following proposition.

Proposition $2.8([21,23])$. If $\mathcal{S} \subset \mathscr{T}^{\mathrm{c}}$ is a set of compact objects that generates $\mathscr{T}$, then the minimal triangulated subcategory of $\mathscr{T}$ containing $\mathcal{S}$ and closed under arbitrary direct sums is $\mathscr{T}$ itself.

If $X$ is a separated $\mathbb{k}$-scheme of finite type and $\mathbf{D}(X)$ is the unbounded derived category of quasicoherent sheaves on $X$, then $\mathrm{D}(X)$ is cocomplete, its subcategory of compact objects coincides with the category of perfect complexes on $X$,

$$
\mathbf{D}(X)^{\mathrm{c}}=\mathbf{D}^{\text {perf }}(X)
$$

and $\mathbf{D}(X)$ is compactly generated (see [22]). Recall that a perfect complex on $X$ is an object of $\mathbf{D}(X)$ that is locally quasiisomorphic to a bounded complex of locally free sheaves of finite rank. In particular, if $X$ is smooth, then

$$
\mathbf{D}(X)^{\mathrm{c}}=\mathbf{D}^{b}(\operatorname{coh}(X)),
$$

the bounded derived category of coherent sheaves. 
Definition 2.9. A functor $\Phi: \mathscr{T}_{1} \rightarrow \mathscr{T}_{2}$ between cocomplete triangulated categories commutes with direct sums if, for any set of objects $G_{i} \in \mathscr{T}_{1}$, the canonical morphism

$$
\oplus \Phi\left(G_{i}\right) \rightarrow \Phi\left(\oplus G_{i}\right)
$$

is an isomorphism. We also say that $\Phi$ preserves compactness if $\Phi\left(\mathscr{T}_{1}^{\mathrm{c}}\right) \subset \mathscr{T}_{2}^{\mathrm{c}}$.

The following simple observation will be used very frequently.

Lemma 2.10. Let $\Phi: \mathscr{T}_{1} \rightarrow \mathscr{T}_{2}$ be a triangulated functor.

(1) Assume that $\Phi$ is fully faithful and commutes with direct sums. If $\Phi(F)$ is compact, then $F$ is compact.

(2) Assume that $\Phi$ has a right adjoint functor $\Phi^{!}$, and $\mathscr{T}_{1}$ is compactly generated. Then, $\Phi$ preserves compactness if and only if $\Phi^{!}$commutes with direct sums.

Proof. (1) Let $F, F_{i} \in \mathscr{T}_{1}$. Consider the following commutative diagram:

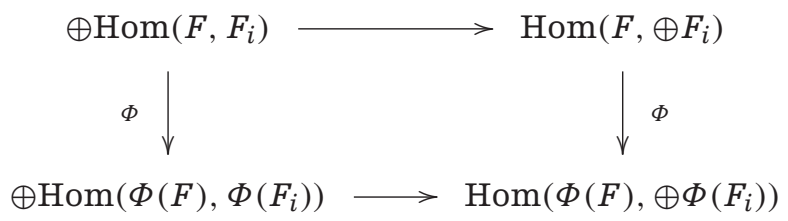

The vertical arrows are isomorphisms since $\Phi$ is fully faithful and commutes with direct sums. The bottom arrow is an isomorphism since $\Phi(F)$ is compact. Hence, the top arrow is an isomorphism, so $F$ is compact.

(2) Let $F \in \mathscr{T}_{1}, G_{i} \in \mathscr{T}_{2}$. Consider the following commutative diagram:

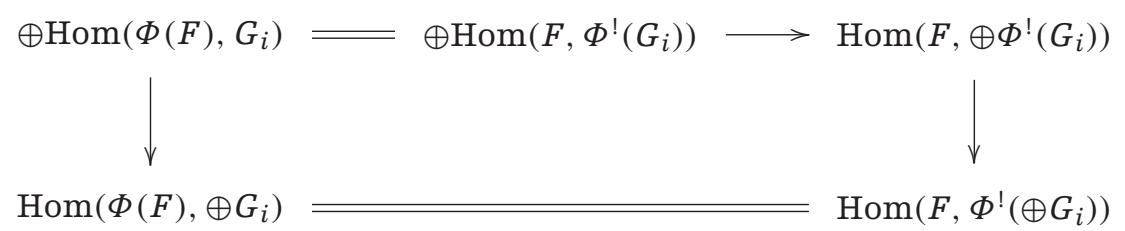

where equalities stand for the adjunction isomorphisms. Let $F$ be compact. Then, the arrow in the top row is an isomorphism. If $\Phi$ preserves compactness, then the left vertical arrow is an isomorphism, hence the right arrow is an isomorphism as well. Since this is true for any compact $F$ and $\mathscr{T}_{1}$ is compactly generated, it follows that the cone 
of the canonical map $\oplus \Phi^{!}\left(G_{i}\right) \rightarrow \Phi^{!}\left(\oplus G_{i}\right)$ is zero, hence $\Phi^{!}$commutes with direct sums. Vice versa, if $\Phi^{!}$commutes with the direct sums, then the right arrow is an isomorphism, hence the left arrow is an isomorphism as well; hence $\Phi(F)$ is compact. Thus, $\Phi$ preserves compactness.

The following result is very useful to ensure the existence of a right adjoint functor.

Theorem 2.11 (Brown representability [22]). Assume that $\Phi: \mathscr{T}_{1} \rightarrow \mathscr{T}_{2}$ is a triangulated functor, $\mathscr{T}_{1}$ is cocomplete and generated by a set of compact objects. Then, $\Phi$ has a right adjoint functor if and only if $\Phi$ commutes with direct sums.

Another useful observation is the following lemma.

Lemma 2.12. Let $\Phi: \mathscr{T} \rightarrow \mathscr{T}^{\prime}$ be a triangulated functor between cocomplete triangulated categories that commutes with arbitrary direct sums. Let $\mathscr{T}_{0} \subset \mathscr{T}$ be a subcategory of compact objects that generates $\mathscr{T}$. If $\Phi$ preserves compactness and is fully faithful on $\mathscr{T}_{0}$, then $\Phi$ is fully faithful on the whole $\mathscr{T}$. If, moreover, $\Phi\left(\mathscr{T}_{0}\right)$ generates $\mathscr{T}^{\prime}$, then $\Phi$ is an equivalence.

Proof. By Brown representability $\Phi$ has a right adjoint functor $\Phi !$. Since $\Phi$ preserves compactness, the adjoint $\Phi^{!}$also commutes with arbitrary direct sums by Lemma 2.10. Consider the adjunction unit id $\rightarrow \Phi^{!} \Phi$ and let $\mathscr{T}_{1} \subset \mathscr{T}$ be the full subcategory consisting of all objects $F$ on which this morphism is an isomorphism. Clearly, it is triangulated and closed under arbitrary direct sums (since both $\Phi$ and $\Phi^{!}$commute with those). Let us show that it contains $\mathscr{T}_{0}$. Indeed, take any $F, G \in \mathscr{T}_{0}$, consider the triangle

$$
G \rightarrow \Phi^{!}(\Phi(G)) \rightarrow G^{\prime}
$$

and apply $\operatorname{Hom}(F,-)$ to it. Since the functor $\Phi$ is full and faithful on $\mathscr{T}_{0}$ we have $\operatorname{Hom}\left(F, \Phi^{!}(\Phi(G))\right) \cong \operatorname{Hom}(\Phi(F), \Phi(G)) \cong \operatorname{Hom}(F, G)$, hence $\operatorname{Hom}\left(F, G^{\prime}\right)=0$. Since this is true for any $F \in \mathscr{T}_{0}$ and $\mathscr{T}_{0}$ generates $\mathscr{T}$, we conclude that $G^{\prime}=0$. Thus, $G \in \mathscr{T}_{1}$. Since this is true for any $G$, we conclude that $\mathscr{T}_{0} \subset \mathscr{T}_{1}$. Now, since $\mathscr{T}_{0}$ generates $\mathscr{T}$ and $\mathscr{T}_{1}$ is closed under arbitrary direct sums, using Proposition 2.8 we conclude that $\mathscr{T}_{1}=\mathscr{T}$, and so $\Phi ! \Phi \cong$ id. This implies that $\Phi$ is fully faithful on the whole $\mathscr{T}$.

Finally, assume that $\Phi\left(\mathscr{T}_{0}\right)$ generates $\mathscr{T}^{\prime}$. Note that $\Phi(\mathscr{T})$ is a full triangulated subcategory of $\mathscr{T}^{\prime}$ (since $\Phi$ is fully faithful), closed under arbitrary direct sums (since $\Phi$ 
commutes with those) and containing $\Phi\left(\mathscr{T}_{0}\right)$. Consequently, by Proposition 2.8 we have $\Phi(\mathscr{T})=\mathscr{T}^{\prime}$, hence $\Phi$ is an equivalence.

\section{Preliminaries on DG-Categories}

A general reference for DG-categories is an excellent review of Keller [14]. See also [10, 27].

\subsection{DG-categories and DG-functors}

A DG-category $\mathscr{D}$ over a field $\mathbb{k}$ is a $\mathbb{k}$-linear category such that

(1) for all $X_{1}, X_{2} \in \mathscr{D}$ the space $\operatorname{Hom}_{\mathscr{D}}\left(X_{1}, X_{2}\right)$ is equipped with a structure of a complex of $\mathbb{k}$-vector spaces, and

(2) the multiplication map

$$
\operatorname{Hom}_{\mathscr{D}}\left(X_{2}, X_{3}\right) \otimes_{\mathbb{k}} \operatorname{Hom}_{\mathscr{D}}\left(X_{1}, X_{2}\right) \rightarrow \operatorname{Hom}_{\mathscr{D}}\left(X_{1}, X_{3}\right)
$$

is a morphism of complexes.

By definition $\operatorname{Hom}_{\mathscr{D}}(X, Y)=\oplus_{k \in \mathbb{Z}} \operatorname{Hom}_{\mathscr{D}}^{k}(X, Y)$ is a graded vector space with a differential $d: \operatorname{Hom}_{\mathscr{D}}^{k}(X, Y) \rightarrow \operatorname{Hom}_{\mathscr{D}}^{k+1}(X, Y)$. The elements $f \in \operatorname{Hom}_{\mathscr{D}}^{k}(X, Y)$ are called homogeneous morphisms of degree $k, \operatorname{deg} f=k$. The second part of the definition of a DGcategory is just the Leibniz rule for the composition of homogeneous morphisms

$$
d(f g)=(d f) g+(-1)^{\operatorname{deg} f} f(d g)
$$

If $\mathscr{D}$ is a DG-category, then the opposite DG-category $\mathscr{D}^{\text {op }}$ is defined as the category with the same objects and $\operatorname{Hom}_{\mathscr{D} \text { op }}\left(X_{1}, X_{2}\right)=\operatorname{Hom}_{\mathscr{D}}\left(X_{2}, X_{1}\right)$ and the composition induced by the composition in $\mathscr{D}$ twisted by the $\operatorname{sign}(-1)^{\operatorname{deg} f \operatorname{deg} g}$.

If $\mathscr{D}_{1}$ and $\mathscr{D}_{2}$ are DG-categories, we define their tensor product $\mathscr{D}_{1} \otimes_{\mathbb{k}} \mathscr{D}_{2}$ as the DG-category with objects $\mathscr{D}_{1} \times \mathscr{D}_{2}$ and morphisms defined by

$$
\operatorname{Hom}_{\mathscr{D}_{1} \otimes_{\mathfrak{k}} \mathscr{D}_{2}}\left(\left(X_{1}, X_{2}\right),\left(Y_{1}, Y_{2}\right)\right)=\operatorname{Hom}_{\mathscr{D}_{1}}\left(X_{1}, Y_{1}\right) \otimes_{\mathbb{k}} \operatorname{Hom}_{\mathscr{D}_{2}}\left(X_{2}, Y_{2}\right)
$$

The simplest example of a DG-category is the category $\mathbb{k}$-dgm of complexes of $\mathbb{k}$-vector spaces with

$$
\operatorname{Hom}^{k}\left(V_{1}, V_{2}\right)=\prod_{i \in \mathbb{Z}} \operatorname{Hom}\left(V_{1}^{i}, V_{2}^{i+k}\right), \quad d(f)=d_{V_{2}} \circ f-(-1)^{\operatorname{deg} f} f \circ d_{V_{1}} .
$$


Also note that each $\mathbb{k}$-linear category can be considered as a DG-category with the same Hom-spaces with zero differential and zero grading.

A $\mathbb{k}$-linear functor $F: \mathscr{D}_{1} \rightarrow \mathscr{D}_{2}$ is a DG-functor if the morphisms

$$
F_{X_{1}, X_{2}}: \operatorname{Hom}_{\mathscr{D}_{1}}\left(X_{1}, X_{2}\right) \rightarrow \operatorname{Hom}_{\mathscr{D}_{2}}\left(F\left(X_{1}\right), F\left(X_{2}\right)\right)
$$

are morphisms of complexes, that is, they preserve the grading and commute with the differentials.

If $\mathscr{D}_{1}$ is a small DG-category (i.e., objects of $\mathscr{D}_{1}$ form a set), then all DG-functors from $\mathscr{D}_{1}$ to $\mathscr{D}_{2}$ form a DG-category $\operatorname{Fun}\left(\mathscr{D}_{1}, \mathscr{D}_{2}\right)$ with $\operatorname{Hom}_{\text {Fun }\left(\mathscr{D}_{1}, \mathscr{D}_{2}\right)}(F, G)$ defined as

$$
\operatorname{Ker}\left(\prod_{X \in \mathscr{D}_{1}} \operatorname{Hom}_{\mathscr{D}_{2}}(F(X), G(X)) \rightarrow \prod_{X, Y \in \mathscr{D}_{1}} \operatorname{Hom}\left(\operatorname{Hom}_{\mathscr{D}_{1}}(X, Y), \operatorname{Hom}_{\mathscr{D}_{2}}(F(X), G(Y))\right)\right)
$$

In other words, an element $f \in \operatorname{Hom}_{\text {Fun }\left(\mathscr{D}_{1}, \mathscr{D}_{2}\right)}^{k}(F, G)$ is a collection of morphisms $f_{X} \in$ $\operatorname{Hom}_{\mathscr{D}_{2}}^{k}(F(X), G(X))$ given for all $X \in \mathscr{D}_{1}$ such that, for any morphism $g \in \operatorname{Hom}_{\mathscr{D}_{1}}^{l}(X, Y)$, one has $f_{Y} \circ F(g)=(-1)^{k l} G(g) \circ f_{X}$. The differential is induced by the differentials in $\operatorname{Hom}_{\mathscr{D}_{2}}(F(X), G(X))$.

A right DG-module over $\mathscr{D}$ is a DG-functor $M: \mathscr{D}^{\mathrm{op}} \rightarrow \mathbb{k}$-dgm. If $\mathscr{D}$ is small, then the category $\mathscr{D}$-dgm of right DG-modules over $\mathscr{D}$ is a DG-category and the Yoneda functor

$$
\mathrm{Y}: \mathscr{D} \rightarrow \mathscr{D} \text {-dgm }, \quad X \mapsto \operatorname{Hom}_{\mathscr{D}}(-, X)
$$

is a DG-functor. The DG-module $Y(X)$ obtained by applying the Yoneda functor to an object $X \in \mathscr{D}$ will be denoted by $\mathrm{Y}^{X}$. It is called representable DG-module, and one says that the object $X$ is the corresponding representing object. One has an analog of the Yoneda Lemma

$$
\operatorname{Hom}_{\mathscr{D}-\operatorname{dgm}}\left(\mathrm{Y}^{X}, M\right) \cong M(X)
$$

for any DG-module $M \in \mathscr{D}$-dgm. In other words, the Yoneda functor is fully faithful, so $\mathscr{D}$ can be considered as a full DG-subcategory in $\mathscr{D}$-dgm.

Analogously, a left DG-module over $\mathscr{D}$ is a DG-functor $\mathscr{D} \rightarrow \mathbb{k}$-dgm. Note that a left $\mathscr{D}$-module is the same as a right $\mathscr{D}^{\mathrm{op}}$-module. So, the Yoneda functor can be considered also in this case

$$
\mathrm{Y}^{\mathrm{op}}: \mathscr{D}^{\mathrm{op}} \rightarrow \mathscr{D}^{\mathrm{op}} \text {-dgm, } \quad X \mapsto \operatorname{Hom}_{\mathscr{D}}(X,-) .
$$

We will write $Y_{X}$ for $Y^{o p}(X)$. 


\subsection{The homotopy category}

The homotopy category [ $\mathscr{D}]$ of a DG-category $\mathscr{D}$ is defined as the category with

(1) $\mathrm{Ob}[\mathscr{D}]=\mathrm{Ob} \mathscr{D}$;

(2) $\operatorname{Hom}_{[\mathscr{D}]}(X, Y)=H^{0}\left(\operatorname{Hom}_{\mathscr{D}}(X, Y)\right)$.

The homotopy category is a $\mathbb{k}$-linear category.

One says that closed morphisms of degree zero $f, g \in \operatorname{Hom}_{\mathscr{D}}(X, Y)$ are homotopic if they induce equal morphisms in [ $\mathscr{D}]$; in other words, if there is $h \in \operatorname{Hom}_{\mathscr{D}}^{-1}(X, Y)$ such that $f-g=d h$ (this $h$ is a homotopy between $f$ and $g$ ). Further, two objects $X$ and $Y$ in $\mathscr{D}$ are homotopic (or homotopy equivalent) if they are isomorphic in [ $\mathscr{D}]$. In other words, if there are closed morphisms of degree zero $f \in \operatorname{Hom}_{\mathscr{D}}(X, Y)$ and $g \in \operatorname{Hom}_{\mathscr{D}}(Y, X)$ such that $f g$ is homotopic to $\mathrm{id}_{Y}$ and $g f$ is homotopic to $\mathrm{id}_{X}$.

Each DG-functor $F: \mathscr{D}_{1} \rightarrow \mathscr{D}_{2}$ induces a functor $[F]:\left[\mathscr{D}_{1}\right] \rightarrow\left[\mathscr{D}_{2}\right]$ on homotopy categories. A DG-functor $F: \mathscr{D}_{1} \rightarrow \mathscr{D}_{2}$ is a quasiequivalence if, for all $X_{1}, Y_{1} \in \mathscr{D}_{1}$, the morphism $F: \operatorname{Hom}_{\mathscr{D}_{1}}\left(X_{1}, Y_{1}\right) \rightarrow \operatorname{Hom}_{\mathscr{D}_{2}}\left(F\left(X_{1}\right), F\left(Y_{1}\right)\right)$ is a quasiisomorphism of complexes (in this case $F$ is called quasifully faithful) and for each object $X_{2} \in \mathscr{D}_{2}$, there is an object $X_{1} \in \mathscr{D}_{1}$ such that $F\left(X_{1}\right)$ is homotopy equivalent to $X_{2}$. In particular, $[F]$ is an equivalence of categories.

Two DG-categories $\mathscr{D}$ and $\mathscr{D}^{\prime}$ are called quasiequivalent if there is a pair of quasiequivalences $\mathscr{D} \stackrel{\cong}{\cong} \stackrel{\mathscr{D}}{\cong} \mathscr{D}^{\prime}$. It is clear that quasiequivalent DG-categories have equivalent homotopy categories.

\subsection{Pretriangulated categories}

Let $\mathscr{D}$ be a DG-category and $M$ be a DG-module over $\mathscr{D}$. For each integer $k \in \mathbb{Z}$ the shift $M[k]$ is defined as the DG-module over $\mathscr{D}$ with

$$
M[k](X)=M(X)[k]
$$

for all $X \in \mathscr{D}$, where the RHS is the usual shift of the complex $M(X)$. Further, let $f: M \rightarrow N$ be a closed morphism of DG-modules of degree 0 . Then, for any object $X \in \mathscr{D}$ we have a morphism of complexes $f_{X}: M(X) \rightarrow N(X)$. The cone of the morphism $f$ is defined by

$$
\text { Cone }(f)(X)=\text { Cone }\left(f_{X}: M(X) \rightarrow N(X)\right)
$$

Again, the RHS is the usual cone of a morphism of complexes. 
Remark 3.1. In other words, the value of Cone $(f)$ on $X \in \mathscr{D}$ is the direct sum of graded vector spaces $N(X) \oplus M(X)[1]$ with the differential $d(n, m)=(d n+f(m),-d m)$. Thus, the cone comes with a bunch of morphisms:

$$
M[1] \stackrel{i}{\rightarrow} \operatorname{Cone}(f) \stackrel{p}{\rightarrow} M[1], \quad N \stackrel{j}{\rightarrow} \operatorname{Cone}(f) \stackrel{s}{\rightarrow} N,
$$

such that

$$
p i=\mathrm{id}_{M[1]}, \quad s j=\mathrm{id}_{N}, \quad i p+j s=\mathrm{id}_{\mathrm{Cone}(f)}, \quad d j=d p=0, \quad d i=j f, \quad d s=-f p .
$$

Vice versa, given a DG-module $C$ with morphisms $M[1] \stackrel{i}{\rightarrow} C \stackrel{p}{\rightarrow} M[1], N \stackrel{j}{\rightarrow} C \stackrel{s}{\rightarrow} N$ satisfying the above conditions it is easy to check that $C$ is isomorphic to the cone of $f$.

Note also that Cone $(M[-1] \stackrel{0}{\longrightarrow} N) \cong M \oplus N$ is the direct sum of DG-modules.

For any small DG-category $\mathscr{D}$ the shift functor and the cone functor defined above induce on the homotopy category [ $\mathscr{D}$-dgm] of DG-modules over $\mathscr{D}$ a structure of a triangulated category [7].

Definition 3.2 ([7]). Let $\mathscr{D}$ be a small DG-category. A DG-subcategory $\mathscr{D}^{\prime} \subset \mathscr{D}$-dgm is pretriangulated if its homotopy category $\left[\mathscr{D}^{\prime}\right]$ is a triangulated subcategory of [ $\mathscr{D}$-dgm]. In particular, a small DG-category $\mathscr{D}$ is pretriangulated if

(1) for any $X \in \mathscr{D}$ and any $k \in \mathbb{Z}$, the DG-module $Y^{X}[k]$ is homotopic to a representable DG-module;

(2) for any closed morphism $f \in \operatorname{Hom}_{\mathscr{D}}\left(X_{1}, X_{2}\right)$ of degree 0 in $\mathscr{D}$, the DG-module Cone $\left(Y^{f}: Y^{X_{1}} \rightarrow Y^{X_{2}}\right)$ is homotopic to a representable DG-module.

The homotopy category of a pretriangulated DG-category is triangulated. Note also that if $F: \mathscr{D}_{1} \rightarrow \mathscr{D}_{2}$ is a DG-functor between small pretriangulated DG-categories, then the functor $[F]:\left[\mathscr{D}_{1}\right] \rightarrow\left[\mathscr{D}_{2}\right]$ is triangulated.

Definition 3.3 ([7]). An enhancement for a triangulated category $\mathscr{T}$ is a pretriangulated DG-category $\mathscr{D}$ with an equivalence $\mathscr{T} \cong[\mathscr{D}]$ of triangulated categories.

\subsection{Derived category}

Note that the category of DG-modules over a DG-category $\mathscr{D}$ has arbitrary direct sums, which are just componentwise

$$
\left(\oplus M_{i}\right)(X)=\oplus M_{i}(X)
$$

for any set of DG-modules $M_{i}$ and any $X \in \mathscr{D}$. 
A DG-module $M$ over $\mathscr{D}$ is acyclic, if for any $X \in \mathscr{D}$, the complex $M(X)$ is acyclic. The DG-subcategory of acyclic DG-modules is denoted by $\operatorname{Acycl}(\mathscr{D})$. It is evidently pretriangulated and closed under arbitrary direct sums, hence its homotopy category $[\operatorname{Acycl}(\mathscr{D})]$ is a localizing triangulated subcategory in [ $\mathscr{D}$-dgm].

The derived category of DG-modules over $\mathscr{D}$ is defined as the Verdier quotient

$$
\mathbf{D}(\mathscr{D})=[\mathscr{D}-\operatorname{dgm}] /[\operatorname{Acycl}(\mathscr{D})]
$$

It is a triangulated category. Two DG-modules are called quasiisomorphic if they are isomorphic in the derived category of DG-modules.

By [21], the functor $[\mathscr{D}$-dgm] $\rightarrow \mathbf{D}(\mathscr{D})$ commutes with direct sums. It follows that the images of representable objects are compact in $\mathbf{D}(\mathscr{D})$. In particular, $\mathbf{D}(\mathscr{D})$ is compactly generated. In fact, compact objects in $\mathbf{D}(\mathscr{D})$ can be described as follows.

Consider the minimal subcategory of $\mathscr{D}$-dgm containing all representable DGmodules and closed under shifts, cones of closed morphisms, and homotopy direct summands. Objects of this category are called perfect DG-modules. By [14, Theorem 3.4], compact objects of $\mathbf{D}(\mathscr{D})$ are given by perfect DG-modules. In particular, if $\mathscr{D}$ is pretriangulated and closed under homotopy direct summands, then

$$
\mathbf{D}(\mathscr{D})^{\mathrm{C}}=[\mathscr{D}]
$$

A DG-module $P$ is called h-projective if, for any acyclic DG-module $A$, the complex $\operatorname{Hom}_{\mathscr{D} \text {-dgm }}(P, A)$ is acyclic. The DG-subcategory of $\mathscr{D}$-dgm consisting of h-projective DGmodules is denoted by h-proj( $\mathscr{D})$. A DG-module $I$ is called h-injective if, for any acyclic DG-module $A$, the complex $\operatorname{Hom}_{\mathscr{D}-\operatorname{dgm}}(A, I)$ is acyclic. The DG-subcategory of $\mathscr{D}$-dgm consisting of h-injective DG-modules is denoted by $\mathrm{h}$-inj( $\mathscr{D})$. Note that by definition $\mathrm{h}-\operatorname{proj}(\mathscr{D})$ is just the left orthogonal to $\operatorname{Acycl}(\mathscr{D})$ in $[\mathscr{D}-\mathrm{dgm}]$, while $\mathrm{h}$-inj( $\mathscr{D})$ is just the right orthogonal. In fact, the following is well known.

Theorem 3.4 ([14, Proposition 3.1]). There are semiorthogonal decompositions

$$
[\mathscr{D}-\operatorname{dgm}]=\langle[\operatorname{Acycl}(\mathscr{D})],[\mathrm{h}-\operatorname{proj}(\mathscr{D})]\rangle, \quad[\mathscr{D}-\operatorname{dgm}]=\langle[\mathrm{h}-\operatorname{inj}(\mathscr{D})],[\operatorname{Acycl}(\mathscr{D})]\rangle
$$

In particular, we have equivalences of triangulated categories

$$
[\mathrm{h}-\operatorname{proj}(\mathscr{D})] \cong \mathbf{D}(\mathscr{D}) \cong[\mathrm{h}-\operatorname{inj}(\mathscr{D})]
$$

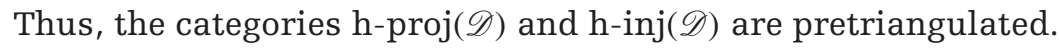


It follows from Theorem 3.4 that each DG-module $M$ is quasiisomorphic to an h-projective DG-module $P_{M}$, which is called its h-projective resolution. Analogously, each DG-module $M$ is quasiisomorphic to an h-injective DG-module $I_{M}$, which is called its h-injective resolution. Thus, the category of h-projective (respectively, h-injective) $\mathscr{D}$ modules provides an enhancement for the derived category.

Note also that the image of the Yoneda functor consists of h-projective $\mathscr{D}$-modules-this follows immediately from (7). Thus, [ $\mathscr{D}]$ can be considered as a full subcategory of $\mathbf{D}(\mathscr{D})$ and $\mathscr{D}$ is pretriangulated if and only if this subcategory is triangulated.

\subsection{Tensor products}

If $\mathscr{D}$ is a small DG-category, the tensor product of a right and a left DG-module, $M \in$ $\mathscr{D}$-dgm and $N \in \mathscr{D}^{\mathrm{op}}$-dgm, is defined as

$$
M \otimes_{\mathscr{D}} N=\operatorname{Coker}\left(\bigoplus_{X, Y \in \mathscr{D}} M(X) \otimes_{\mathbb{k}} \operatorname{Hom}_{\mathscr{D}}(Y, X) \otimes_{\mathbb{k}} N(Y) \longrightarrow \bigoplus_{X \in \mathscr{D}} M(X) \otimes_{\mathbb{k}} N(X)\right) .
$$

It is a complex of vector spaces. One checks immediately that

$$
\mathrm{Y}^{X} \otimes_{\mathscr{D}} N=N(X), \quad M \otimes_{\mathscr{D}} \mathrm{Y}_{X}=M(X)
$$

A DG-module $F$ is called h-flat if for any acyclic left DG-module $A$, the tensor product $F \otimes_{\mathscr{D}} A$ is acyclic. It follows from (9) that each representable DG-module is h-flat. Also one can check that each h-projective DG-module is h-flat.

The derived tensor product is defined by using h-flat resolutions of either of the factors

$$
M \stackrel{\mathbb{L}}{\otimes} N:=F_{M} \otimes_{\mathscr{D}} N \cong F_{M} \otimes_{\mathscr{D}} F_{N} \cong M \otimes_{\mathscr{D}} F_{N}
$$

where $F_{M}$ and $F_{N}$ are h-flat resolutions of $M$ and $N$, respectively (e.g., one can use hprojective resolutions). By definition the derived tensor product is defined up to a quasiisomorphism.

Since, as we already mentioned, each representable DG-module is h-flat, we conclude that

$$
\mathrm{Y}^{X} \stackrel{\mathbb{L}}{\otimes} \mathscr{D}=N(X), \quad M \stackrel{\mathbb{L}}{\otimes} \mathrm{Y}_{X}=M(X)
$$




\subsection{Bimodules}

Let $\mathscr{D}_{1}$ and $\mathscr{D}_{2}$ be DG-categories. A $\mathscr{D}_{1}-\mathscr{D}_{2}$ DG-bimodule is a DG-module over $\mathscr{D}_{1}^{\mathrm{op}} \otimes \mathscr{D}_{2}$, that is, a DG-functor $\mathscr{D}_{1} \otimes \mathscr{D}_{2}^{\mathrm{op}} \rightarrow \mathbb{k}$-dgm.

One says that a DG-bimodule $\varphi \in \mathscr{D}_{1}^{\mathrm{op}} \otimes \mathscr{D}_{2}$-dgm has some property as a left (right) DG-module, if, for each $X_{2} \in \mathscr{D}_{2}$ (respectively, for each $X_{1} \in \mathscr{D}_{1}$ ), DG-module $\varphi\left(-, X_{2}\right) \in \mathscr{D}_{1}^{\text {op }}$-dgm (respectively, $\varphi\left(X_{1},-\right) \in \mathscr{D}_{2}$-dgm) has this property. In particular, we can say that a bimodule is representable (h-projective, h-flat, h-injective, ...) as a left (right) DG-module (for short left representable, h-projective, ...).

Given a left DG-module $M_{1}$ over $\mathscr{D}_{1}$ and a right DG-module $M_{2}$ over $\mathscr{D}_{2}$ one defines their exterior tensor product as

$$
\left(M_{1} \otimes_{\mathbb{k}} M_{2}\right)\left(X_{1}, X_{2}\right):=M_{1}\left(X_{1}\right) \otimes_{\mathbb{k}} M_{2}\left(X_{2}\right) .
$$

Clearly, this is a DG-bimodule. Moreover, it is clear that an exterior product of two representable bimodules is representable

$$
Y_{X_{1}} \otimes_{\mathbb{k}} Y^{X_{2}} \cong Y^{\left(X_{1}, X_{2}\right)}
$$

One can check that exterior tensor product of h-projective DG-modules is an $\mathrm{h}$ projective DG-bimodule. Vice versa, an h-projective DG-bimodule is both left and right h-projective. Also one can check that an h-flat DG-bimodule is both left and right h-flat.

Let $\varphi_{12} \in\left(\mathscr{D}_{1}^{\mathrm{op}} \otimes \mathscr{D}_{2}\right)$-dgm and $\varphi_{23} \in\left(\mathscr{D}_{2}^{\mathrm{op}} \otimes \mathscr{D}_{3}\right)$-dgm be two DG-bimodules. Their tensor product is the $\mathscr{D}_{1}-\mathscr{D}_{3}$-DG-bimodule defined by

$$
\left(\varphi_{12} \otimes_{\mathscr{D}_{2}} \varphi_{23}\right)\left(X_{1}, X_{3}\right)=\varphi_{12}\left(X_{1},-\right) \otimes_{\mathscr{D}_{2}} \varphi_{23}\left(-, X_{3}\right)
$$

for all $X_{1} \in \mathscr{D}_{1}$ and $X_{3} \in \mathscr{D}_{3}$.

Analogously, the derived tensor product of bimodules is defined by replacing the first bimodule with a right $\mathrm{h}$-flat resolution or the second with a left h-flat resolution.

For each DG-category $\mathscr{D}$ we denote by $\mathscr{D}$ the diagonal bimodule

$$
\mathscr{D}\left(X_{1}, X_{2}\right)=\operatorname{Hom}_{\mathscr{D}}\left(X_{2}, X_{1}\right) \in \mathscr{D}^{\mathrm{op}} \otimes \mathscr{D} \text {-dgm }
$$

Remark 3.5. Note that the positions of $X_{1}$ and $X_{2}$ in the left-hand and in the right-hand sides of the formula are interchanged. 
It is clear that the diagonal bimodule is both left and right representable. Moreover, it follows immediately from (9) that

$$
M_{1} \otimes_{\mathscr{D}} \mathscr{D} \cong M_{1}, \quad \mathscr{D} \otimes_{\mathscr{D}} M_{2} \cong M_{2}
$$

for all $M_{1} \in \mathscr{D}_{1}$-dgm and $M_{2} \in \mathscr{D}_{2}^{\mathrm{op}}$-dgm. In particular, the diagonal bimodule is both left and right h-flat (but not h-flat as a bimodule), so one can derive freely the tensor product in the above formulas:

$$
M_{1} \stackrel{\mathbb{L}}{\otimes} \mathscr{D} \mathscr{D} \cong M_{1}, \quad \mathscr{D} \stackrel{\mathbb{L}}{\otimes} M_{2} \cong M_{2}
$$

Let $\varphi \in \mathscr{D}_{1}^{\mathrm{op}} \otimes \mathscr{D}_{2}$-dgm be a DG-bimodule. If $F: \mathscr{D}_{1}^{\prime} \rightarrow \mathscr{D}_{1}$ and $G: \mathscr{D}_{2}^{\prime} \rightarrow \mathscr{D}_{2}$ are DGfunctors, we define ${ }_{F} \varphi_{G}$ to be a $\mathscr{D}_{1}^{\prime}-\mathscr{D}_{2}^{\prime}$ DG-bimodule defined by

$$
{ }_{F} \varphi_{G}\left(X_{1}^{\prime}, X_{2}^{\prime}\right)=\varphi\left(F\left(X_{1}^{\prime}\right), G\left(X_{2}^{\prime}\right)\right)
$$

In particular, if $F: \mathscr{D}^{\prime} \rightarrow \mathscr{D}$ is a DG-functor, we have DG-bimodules ${ }_{F} \mathscr{D}_{,} \mathscr{D}_{F}$, and $\mathscr{D}_{F}$.

\subsection{Smoothness and properness}

As we already mentioned in Section 1, a small DG-category $\mathscr{D}$ is smooth if the diagonal bimodule $\mathscr{D}$ is a perfect bimodule. In other words, if $\mathscr{D}$ is a homotopy direct summand of a bimodule obtained from representative bimodules by finite number of shifts and cones of closed morphisms.

A small DG-category is proper if, for all objects $X, Y \in \mathscr{D}$, the complex $\mathscr{D}(X, Y)$ has bounded and finite-dimensional cohomology.

We will need the following result of Toën and Vaquié, which allows one to prove the perfectness of a bimodule. Let $\varphi \in\left(\mathscr{D}_{1} \otimes \mathscr{D}_{2}^{\mathrm{op}}\right)$-dgm be a DG-bimodule. Consider the derived tensor product functor

$$
L \varphi: \mathbf{D}\left(\mathscr{D}_{2}\right) \rightarrow \mathbf{D}\left(\mathscr{D}_{1}\right), \quad L \varphi(-)=-\stackrel{\mathbb{L}}{\otimes} \mathscr{D}_{2} \varphi
$$

This is a triangulated functor commuting with arbitrary direct sums.

Proposition 3.6 ([26, Lemma 2.8.2]). If DG-category $\mathscr{D}_{2}$ is smooth and the derived tensor product functor $L \varphi: \mathbf{D}\left(\mathscr{D}_{2}\right) \rightarrow \mathbf{D}\left(\mathscr{D}_{1}\right)$ preserves compactness, then $\varphi$ is a perfect DG-bimodule. 


\subsection{Drinfeld quotient}

Let $\mathscr{D}$ be a DG-category and $\mathscr{D}_{0} \subset \mathscr{D}$ be its small full DG-subcategory. In [10], Drinfeld defined a new DG-category $\mathscr{D} / \mathscr{D}_{0}$, which is known as Drinfeld quotient. By definition $\mathscr{D} / \mathscr{D}_{0}$ has the same objects as $\mathscr{D}$ and $\mathrm{Hom}_{\mathscr{D} / \mathscr{D}_{0}}$ is freely generated over Hom $\mathscr{D}$ by generators $\varepsilon_{X}$, one for each object $X$ of $\mathscr{D}_{0}$, such that

$$
\operatorname{deg} \varepsilon_{X}=-1, \quad d\left(\varepsilon_{X}\right)=1_{X} .
$$

Thus, $\operatorname{Hom}_{\mathscr{D} / \mathscr{D}_{0}}\left(Y, Y^{\prime}\right)$ is given by

$$
\bigoplus_{p=0}^{\infty} \bigoplus_{X_{1}, \ldots, X_{p} \in \mathscr{D}_{0}} \operatorname{Hom}_{\mathscr{D}}\left(Y, X_{1}\right) \otimes \varepsilon_{X_{1}} \otimes \operatorname{Hom}_{\mathscr{D}}\left(X_{1}, X_{2}\right) \otimes \varepsilon_{X_{2}} \otimes \cdots \otimes \varepsilon_{X_{p}} \otimes \operatorname{Hom}_{\mathscr{D}}\left(X_{p}, Y^{\prime}\right) .
$$

The Drinfeld quotient $\mathscr{D} / \mathscr{D}_{0}$ comes with a natural DG-functor $\eta_{\mathscr{D}, \mathscr{D}_{0}}: \mathscr{D} \rightarrow \mathscr{D} / \mathscr{D}_{0}$, which takes $\operatorname{Hom}_{\mathscr{D}}\left(Y, Y^{\prime}\right)$ to the $p=0$ summand above. The following nice property of the Drinfeld quotient follows from the definition.

Proposition 3.7. Let $\mathscr{D}, \mathscr{D}^{\prime}$ be DG-categories and $\mathscr{D}_{0} \subset \mathscr{D}, \mathscr{D}_{0}^{\prime} \subset \mathscr{D}^{\prime}$ their DG-subcategories. If $F: \mathscr{D} \rightarrow \mathscr{D}^{\prime}$ is a DG-functor such that $F\left(\mathscr{D}_{0}\right) \subset \mathscr{D}_{0}^{\prime}$, then there is a DG-functor $\bar{F}: \mathscr{D} / \mathscr{D}_{0} \rightarrow$ $\mathscr{D}^{\prime} / \mathscr{D}_{0}^{\prime}$ such that the diagram

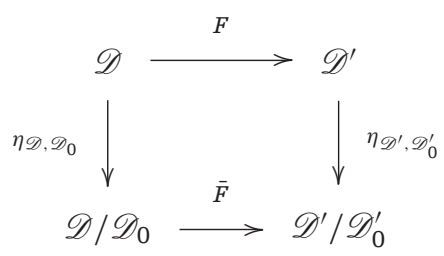

commutes, that is, $\bar{F} \circ \eta_{\mathscr{D}, \mathscr{D}_{0}}=\eta_{\mathscr{D}^{\prime}, \mathscr{O}_{0}^{\prime}} \circ F$.

Proof. We define $\bar{F}$ to be the same as $F$ on objects, and to extend it on morphisms we define $\bar{F}\left(\varepsilon_{X}\right)=\varepsilon_{F(X)}$. By definition of $\mathscr{D} / \mathscr{D}_{0}$ this uniquely defines $\bar{F}$.

The definition of the Drinfeld quotient $\mathscr{D} / \mathscr{D}_{0}$ as presented above makes sense only in case when $\mathscr{D}_{0}$ is a small DG-category. Otherwise, the Hom-spaces defined by (14) are not sets. However, one can use the machinery of Grothendieck universes to define the Drinfeld quotient for arbitrary $\mathscr{D}_{0}$. We skip the details and refer the interested reader 
to the Appendix in [19]. Note, by the way, that the Drinfeld quotient provides a natural enhancement for the Verdier quotient.

Theorem 3.8 ([10]). If $\mathscr{D}$ is a pretriangulated DG-category and $\mathscr{D}_{0} \subset \mathscr{D}$ is its pretriangulated DG-subcategory, then $\mathscr{D} / \mathscr{D}_{0}$ is also pretriangulated and there is an equivalence of triangulated categories $\left[\mathscr{D} / \mathscr{D}_{0}\right] \cong[\mathscr{D}] /\left[\mathscr{D}_{0}\right]$.

\subsection{Extensions of DG-functors}

Let $F: \mathscr{D}_{1} \rightarrow \mathscr{D}_{2}$ be a DG-functor between small DG-categories. For each right DG-module $M$ over $\mathscr{D}_{2}$ we have a DG-module $M_{F}(X)=M(F(X))$ over $\mathscr{D}_{1}$. This defines a DG-functor of restriction $\operatorname{Res}_{F}: \mathscr{D}_{2}$-dgm $\rightarrow \mathscr{D}_{1}$-dgm which evidently takes acyclic DG-modules to acyclic DG-modules and so descends to a functor between derived categories which we also define as

$$
\operatorname{Res}_{F}: \mathbf{D}\left(\mathscr{D}_{2}\right) \rightarrow \mathbf{D}\left(\mathscr{D}_{1}\right), \quad M \mapsto M_{F}
$$

On the other hand, the DG-functor $F$ produces a $\mathscr{D}_{1}-\mathscr{D}_{2}$-bimodule ${ }_{F} \mathscr{D}_{2}$, so one can define the induction functor $\operatorname{Ind}_{F}: \mathscr{D}_{1}$-dgm $\rightarrow \mathscr{D}_{2}$-dgm, $N \mapsto N \otimes_{\mathscr{D}_{1} F} \mathscr{D}_{2}$ as well as its derived functor

$$
\operatorname{LInd}_{F}: \mathbf{D}\left(\mathscr{D}_{1}\right) \rightarrow \mathbf{D}\left(\mathscr{D}_{2}\right), \quad N \mapsto N \stackrel{\mathbb{L}}{\otimes} \mathscr{D}_{1 F} \mathscr{D}_{2}
$$

Proposition 3.9. The derived induction functor $\operatorname{LInd}_{F}$ is left adjoint to the restriction functor $\operatorname{Res}_{F}$. Both functors Res R $_{F}$ and LInd ${ }_{F}$ commute with arbitrary direct sums. Moreover,

$$
\operatorname{LInd}_{F}\left(\mathrm{Y}^{X}\right) \cong \mathrm{Y}^{F(X)}
$$

If $[F]$ is fully faithful, then $\operatorname{LInd}_{F}$ is fully faithful. Finally, if $F$ is a quasiequivalence, then both $\operatorname{Res}_{F}$ and LInd $F$ are equivalences.

Proof. First of all, note that $\operatorname{Res}_{F}$ commutes with arbitrary direct sums by definition and $\operatorname{LInd}_{F}$ commutes since a tensor product does. Further, the formula for $\operatorname{LInd}_{F}\left(\mathrm{Y}^{X}\right)$ follows immediately from (10).

To prove the adjunction, note that the right adjoint of $\operatorname{LInd}_{F}$ exists by Brown representability. Let us denote it temporarily by $G$. Note that, for any $\mathscr{D}_{2}$-module $M$, 
we have

$$
\begin{aligned}
G(M)(X) & \cong \operatorname{Hom}_{\mathbf{D}\left(\mathscr{D}_{1}\right)}\left(\mathrm{Y}^{X}, G(M)\right) \cong \operatorname{Hom}_{\mathbf{D}\left(\mathscr{D}_{2}\right)}\left(\operatorname{LInd}\left(\mathrm{Y}^{X}\right), M\right) \\
& \cong \operatorname{Hom}_{\mathbf{D}\left(\mathscr{D}_{2}\right)}\left(\mathrm{Y}^{F(X)}, M\right) \cong M(F(X)) \cong M_{F}(X)
\end{aligned}
$$

which shows that $G$ is isomorphic to the restriction functor $\operatorname{Res}_{F}$.

Finally, to show that LInd ${ }_{F}$ is fully faithful, we use Lemma 2.12 with $\mathscr{T}=\mathbf{D}\left(\mathscr{D}_{1}\right)$, $\mathscr{T}^{\prime}=\mathbf{D}\left(\mathscr{D}_{2}\right)$, and $\mathscr{T}_{0}=[\mathscr{D}]$, and the same lemma shows that $\operatorname{LInd}_{F}$ is an equivalence if $F$ is a quasiequivalence.

In fact, the functors $\left(\operatorname{Ind}_{F}, \operatorname{Res}_{F}\right.$ ) form what is called a DG-adjoint pair.

\subsection{Derived category of quasicoherent sheaves}

Let $S$ be a separated scheme of finite type. Denote by $\operatorname{Qcoh}(S)$ the abelian category of quasicoherent sheaves on $S$. The derived category $\mathbf{D}(S)$ is defined as the Verdier quotient

$$
\mathbf{D}(S):=[\operatorname{com}(S)] /\left[\operatorname{com}^{\circ}(S)\right]
$$

where $\operatorname{com}(S)$ is the DG-category of complexes over $\operatorname{Qcoh}(S)$ and $\operatorname{com}^{\circ}(S) \subset \operatorname{com}(S)$ is the DG-category of acyclic complexes. However, for our purposes another description of the derived category is more convenient.

Recall that a complex of quasicoherent sheaves $F$ is h-flat if, for any acyclic complex $A$, the complex $\operatorname{Tot}^{\oplus}\left(F \otimes_{\mathcal{O}_{S}} A\right)$ (the direct sum totalization of the bicomplex $F \otimes_{\mathcal{O}_{S}} A$ ) is acyclic. By [1, Proposition 1.1], there are enough h-flat complexes in $\operatorname{com}(S)$ (that is each complex is quasiisomorphic to an h-flat complex), hence there is an equivalence

$$
\mathbf{D}(S) \cong[\text { h-flat }(S)] /\left[\text { h-flat }^{\circ}(S)\right]
$$

where h-flat $(S)$ is the category of h-flat complexes and h-flat ${ }^{\circ}(S) \subset \mathrm{h}$-flat $(S)$ is the category of acyclic h-flat complexes. Using Theorem 3.8, this can be rewritten as the homotopy category of the Drinfeld quotient

$$
\mathbf{D}(S) \cong\left[\text { h-flat }(S) / \text { h-flat }^{\circ}(S)\right]
$$

This definition is especially useful when one is interested in the derived pullback and tensor product functors because of the following observation of Spaltenstein. 
Lemma 3.10 ([24]). For any morphism of schemes $f: T \rightarrow S$ the termwise pullback functor $f^{*}: \operatorname{com}(S) \rightarrow \operatorname{com}(T)$ takes h-flat complexes to h-flat complexes and h-flat acyclic complexes to h-flat acyclic complexes. The tensor product of an h-flat acyclic complex with any complex is acyclic.

This lemma shows that, for a morphism $f: T \rightarrow S$, the pullback functor $f^{*}$ induces a DG-functor

$$
f^{*}: \text { h-flat }(S) \rightarrow \text { h-flat }(T) \quad \text { such that } \quad f^{*}\left(\text { h-flat }^{\circ}(S)\right) \subset \text { h-flat }{ }^{\circ}(T)
$$

Consequently, by Proposition 3.7 it induces a DG-functor of Drinfeld quotients

$$
f^{*}: \text { h-flat }(S) / \text { h-flat }^{\circ}(S) \rightarrow \text { h-flat }(T) / \text { h-flat }^{\circ}(T) .
$$

The induced functor on the homotopy categories is the derived pullback functor

$$
L f^{*}: \mathbf{D}(S) \rightarrow \mathbf{D}(T)
$$

Analogously, one defines the derived tensor product functor.

Note that by construction the derived pullback functor commutes with direct sums. Therefore, by Brown Representability (Theorem 2.11) it has a right adjoint functor

$$
R f_{*}: \mathbf{D}(T) \rightarrow \mathbf{D}(S)
$$

Let Sch be the category of separated schemes of finite type over $\mathbb{k}$ and Tria be the 2 -category of $\mathbb{k}$-linear triangulated categories. Associating with a scheme $S$ its derived category $\mathbf{D}(S)$ and with each morphism of schemes $f: T \rightarrow S$ its derived pullback functor $L f^{*}: \mathbf{D}(S) \rightarrow \mathbf{D}(T)$ defines a pseudofunctor

$$
\mathbf{D}: \text { Sch }^{\text {op }} \rightarrow \text { Tria }, \quad S \mapsto \mathbf{D}(S), \quad f:(T \rightarrow S) \mapsto\left(L f^{*}: \mathbf{D}(S) \rightarrow \mathbf{D}(T)\right),
$$

which we will call the derived category pseudofunctor. In the next section, we will show that it factors through the 2-category of small DG-categories.

\subsection{DG-enhancements for schemes}

Let sDG denote the 2-category of small DG-categories over $\mathbb{k}$. Associating with a small DG-category $\mathscr{D}$ its derived category $\mathbf{D}(\mathscr{D})$ and with a DG-functor $F: \mathscr{D}_{1} \rightarrow \mathscr{D}_{2}$ its derived 
induction functor $\operatorname{LInd}_{F}: \mathbf{D}\left(\mathscr{D}_{1}\right) \rightarrow \mathbf{D}\left(\mathscr{D}_{2}\right)$ gives a pseudofunctor $\mathbf{D}:$ sDG $\rightarrow$ Tria. The main result of this section is the following theorem.

Theorem 3.11. There is a pseudofunctor $\mathscr{D}: \mathrm{Sch}^{\mathrm{op}} \rightarrow \mathrm{sDG}$ such that the diagram

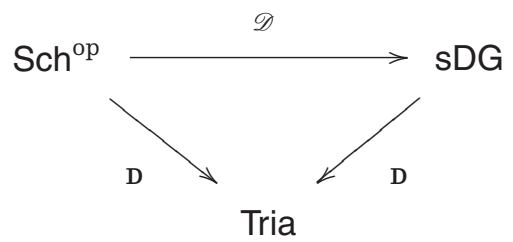

is commutative.

Proof. We want to associate with any scheme $S$ a small DG-category $\mathscr{D}(S)$ and with any morphism of schemes $f: T \rightarrow S$ a DG-functor $f^{*}: \mathscr{D}(S) \rightarrow \mathscr{D}(T)$. Let h-flat-perf $(S)$ be the full DG-subcategory of h-flat $(S)$ consisting of h-flat perfect complexes. Then h-flat-perf $(S) /$ h-flat $^{\circ}(S)$ is a full DG-subcategory in h-flat $(S) /$ h-flat $^{\circ}(S)$. Note that its homotopy category is the subcategory $\mathbf{D}^{\text {perf }}(S) \subset \mathbf{D}(S)$ of perfect complexes. This category is essentially small. Choose a small DG-subcategory $\mathscr{D}(S) \subset \mathrm{h}$-flat-perf $(S) / \mathrm{h}^{-f l a t}{ }^{\circ}(S)$ such that

$$
[\mathscr{D}(S)]=\left[\text { h-flat-perf }(S) / \text { h-flat }^{\circ}(S)\right]=\mathrm{D}^{\text {perf }}(S)
$$

and such that, for any morphism of schemes $f: T \rightarrow S$, we have

$$
f^{*}(\mathscr{D}(S)) \subset \mathscr{D}(T)
$$

For this, we can first take $\mathscr{D}_{0}(S)$ to be just an arbitrary choice of a small DG-subcategory $\mathscr{D}_{0}(S) \subset$ h-flat-perf $(S) /$ h-flat $^{\circ}(S)$ for which (16) holds and then replace it by

$$
\mathscr{D}(S):=\bigcup_{g: S \rightarrow S^{\prime}} g^{*}\left(\mathscr{D}_{0}\left(S^{\prime}\right)\right)
$$

Here, the union is taken with respect to the set of all isomorphism classes of morphisms of separated schemes of finite type (two morphisms $g^{\prime}: S \rightarrow S^{\prime}$ and $g^{\prime \prime}: S \rightarrow S^{\prime \prime}$ are isomorphic if there is an isomorphism $s: S^{\prime} \rightarrow S^{\prime \prime}$ such that $\left.g^{\prime \prime}=s \circ g^{\prime}\right)$. This is indeed a set since the isomorphism classes of separated schemes of finite type form a set and, for a given scheme $S^{\prime}$, all morphisms $S \rightarrow S^{\prime}$ also form a set (a morphism is determined by its graph). 
Then

$$
f^{*}(\mathscr{D}(S))=f^{*}\left(\bigcup_{g: S \rightarrow S^{\prime}} g^{*}\left(\mathscr{D}_{0}\left(S^{\prime}\right)\right)\right)=\bigcup_{g: S \rightarrow S^{\prime}} f^{*}\left(g^{*}\left(\mathscr{D}_{0}\left(S^{\prime}\right)\right)\right) \subset \mathscr{D}(T)
$$

since $f^{*}\left(g^{*}\left(\mathscr{D}_{0}\left(S^{\prime}\right)\right)=(f \circ g)^{*}\left(\mathscr{D}_{0}\left(S^{\prime}\right)\right) \subset \mathscr{D}(T)\right.$.

Now let $f: T \rightarrow S$ be a morphism of separated schemes of finite type. Restricting the DG-functor $f^{*}:$ h-flat $(S) /$ h-flat $^{\circ}(S) \rightarrow$ h-flat $(T) /$ h-flat ${ }^{\circ}(T)$ defined in the previous section to $\mathscr{D}(S)$ and using compatibility (17), we obtain a DG-functor

$$
f^{*}: \mathscr{D}(S) \rightarrow \mathscr{D}(T)
$$

This defines the pseudofunctor on morphisms.

It remains to prove that the diagram of the theorem commutes. In other words, we have to check that, for each scheme $S$, we have an equivalence of categories

$$
\Psi_{S}: \mathbf{D}(\mathscr{D}(S)) \stackrel{\cong}{\longrightarrow} \mathbf{D}(S)
$$

and that the diagram

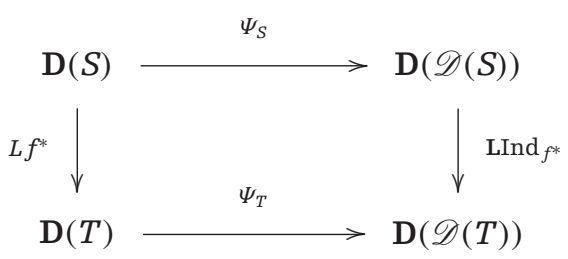

commutes.

Let us start with the equivalence. Recall that $\mathbf{D}(S)=\left[\mathrm{h}-\mathrm{flat}(S) / \mathrm{h}^{-}-\mathrm{flat}^{\circ}(S)\right]$ and that $\mathscr{D}(S)$ is a small DG-subcategory in h-flat $(S) /$ h-flat $^{\circ}(S)$. Define a DG-functor

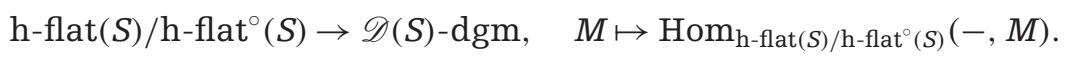

Consider the composition

$$
\Psi_{S}: \mathbf{D}(S)=\left[\mathrm{h}-\text { flat }(S) / \text { h-flat }^{\circ}(S)\right] \rightarrow[\mathscr{D}(S)-\operatorname{dgm}] \rightarrow \mathbf{D}(\mathscr{D}(S))
$$

This functor commutes with arbitrary direct sums since all objects in $\mathscr{D}(S)$ correspond to compact objects in $\mathbf{D}(S)$. Moreover, by definition $\Psi_{S}(M) \cong Y^{M} \in \mathscr{D}(S)$-dgm for any 
perfect complex $M$, hence $\Psi_{S}$ preserves compactness and $\Psi_{S}(\mathscr{D}(S))$ generates $\mathbf{D}(\mathscr{D}(S))$. Finally, for all $M, N \in \mathscr{D}(S)$, we have

$$
\begin{aligned}
\operatorname{Hom}_{\mathbf{D}(\mathscr{D}(S))}\left(\Psi_{S}(M), \Psi_{S}(N)\right) & \cong \operatorname{Hom}_{\mathbf{D}(\mathscr{D}(S))}\left(\mathrm{Y}^{M}, \mathrm{Y}^{N}\right) \\
& \cong \operatorname{Hom}_{[\mathscr{D}(S)]}(M, N) \cong \operatorname{Hom}_{\mathbf{D}(S)}(M, N),
\end{aligned}
$$

hence $\Psi_{S}$ is fully faithful on $[\mathscr{D}(S)]$. Applying Lemma 2.12, we conclude that $\Psi_{S}$ is an equivalence.

It remains to check that the diagram commutes. Let us first construct a morphism of functors $\operatorname{LInd}_{f^{*}} \circ \Psi_{S} \rightarrow \Psi_{T} \circ L f^{*}$. The right adjoint of the derived induction functor is the restriction functor, so it suffices to construct a morphism of functors $\Psi_{S} \rightarrow$ $\operatorname{Res}_{f^{*}} \circ \Psi_{T} \circ L f^{*}$. Both parts are induced by DG-functors h-flat $(S) / \mathrm{h}-\mathrm{flat}^{\circ}(S) \rightarrow \mathscr{D}(S)$-dgm, the first by

$$
M \mapsto \operatorname{Hom}_{\mathrm{h}-\mathrm{flat}(S) / \mathrm{h}-\mathrm{flat}^{\circ}(S)}(-, M)
$$

and the second by

$$
M \mapsto \operatorname{Hom}_{\mathrm{h}-\mathrm{flat}(T) / \mathrm{h}-\mathrm{flat}^{\circ}(T)}\left(f^{*}(-), f^{*}(M)\right)
$$

The DG-functor $f^{*}$ gives a morphism from the first to the second, which on passing to homotopy categories induces the required morphism of functors. Let us check that it is an isomorphism.

Let $\mathscr{T} \subset \mathbf{D}(S)$ be the full subcategory of $\mathbf{D}(S)$ formed by objects $M$ such that the

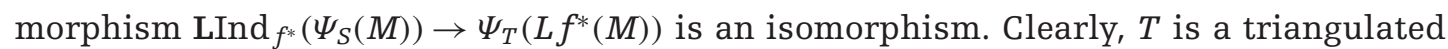
subcategory. Moreover, it contains all objects from $[\mathscr{D}(S)] \subset \mathbf{D}(S)$. Indeed, if $M \in \mathscr{D}(S)$, then

$$
\operatorname{LInd}_{f^{*}}\left(\Psi_{S}(M)\right) \cong \operatorname{LInd}_{f^{*}}\left(Y^{M}\right) \cong Y^{f^{*}(M)} \cong \Psi_{T}\left(f^{*}(M)\right) \cong \Psi_{T}\left(L f^{*}(M)\right)
$$

so $M \in \mathscr{T}$. Finally, $\mathscr{T}$ is closed under arbitrary direct sums, since all the functors $\Psi_{S}, \Psi_{T}$, $L f^{*}$, and $\operatorname{LInd}_{f^{*}}$ commute with those. By Proposition 2.8, we conclude that $\mathscr{T}=\mathbf{D}(S)$, so the diagram is commutative.

Note that, for a separated scheme $S$ of finite type over a field, the enhancement $\mathscr{D}(S)$ of the category $\mathrm{D}^{\text {perf }}(S)$ of perfect complexes on $S$ is smooth (see Definition 1.1) if $S$ is smooth and proper if $S$ is proper by [26, Lemma 3.27]. 


\subsection{From a DG-resolution to a categorical resolution}

Recall the definition 1.5 of a partial DG-resolution.

Lemma 3.12. If $\pi: \mathscr{D} \rightarrow \mathscr{D}^{\prime}$ and $\pi^{\prime}: \mathscr{D}^{\prime} \rightarrow \mathscr{D}^{\prime \prime}$ are partial DG-resolutions, then the composition $\pi^{\prime} \circ \pi: \mathscr{D} \rightarrow \mathscr{D}^{\prime \prime}$ is a partial DG-resolution. Moreover, if in addition $\pi^{\prime}$ is a DGresolution, then so is $\pi^{\prime} \circ \pi$.

Proof. The proof evidently follows from the definition.

If for a scheme $Y$ a DG-resolution of the DG-category $\mathscr{D}(Y)$ is given, we can construct a categorical resolution of $Y$. For this, we use Proposition 3.9 to induce the functors on the derived categories.

Proposition 3.13. Let $\mathscr{D}$ be a small pretriangulated DG-category and $\pi: \mathscr{D}(Y) \rightarrow \mathscr{D}$ be a DG-resolution. Let $\pi^{*}=\operatorname{LInd}_{\pi}: \mathbf{D}(Y)=\mathbf{D}(\mathscr{D}(Y)) \rightarrow \mathbf{D}(\mathscr{D})$ be the derived induction and let $\pi_{*}=\operatorname{Res}_{\pi}: \mathbf{D}(\mathscr{D}) \rightarrow \mathbf{D}(Y)$ be the restriction functors. If $\pi_{*}([\mathscr{D}]) \subset \mathbf{D}^{b}(\operatorname{coh}(Y))$, then $\mathbf{D}(\mathscr{D})$ is a categorical resolution of $\mathbf{D}^{b}(\operatorname{coh}(Y))$.

Proof. By Proposition 3.9, we know that $\pi^{*}$ is fully faithful, commutes with direct sums, and has a right adjoint functor $\pi_{*}$ also commuting with direct sums. So the first two conditions of Definition 1.3 are satisfied. Since $\mathbf{D}(\mathscr{D})^{\mathrm{C}}=[\mathscr{D}]$, the third condition is satisfied as well.

Remark 3.14. If $Y$ is projective and $\mathscr{D}$ is proper, then one can check that the condition $\pi_{*}([\mathscr{D}]) \subset \mathbf{D}^{b}(\operatorname{coh}(Y))$ holds automatically. Indeed, in this case the subcategory $\mathbf{D}^{b}(\operatorname{coh}(Y)) \subset \mathbf{D}(Y)$ consists of all objects $F$ such that $\oplus_{i} \operatorname{Hom}(P, F[i])$ is finite-dimensional for all perfect $P$. But by adjunction

$$
\oplus_{i} \operatorname{Hom}\left(P, \pi_{*}(G)[i]\right) \cong \oplus_{i} \operatorname{Hom}\left(\pi^{*}(P), G[i]\right)
$$

is finite-dimensional for all $G \in \mathscr{D}$ since $\pi^{*}(P) \cong \pi(P)$ is representable and DG-category $\mathscr{D}$ is proper.

\section{The Gluing}

The notion of the gluing of two DG-categories is well known to experts. One possible definition may be found in [25] under the name of upper triangular DG-categories. We 
prefer to use a slightly different definition as it is more adjusted to our goals. Below we will discuss its relation to the definition of Tabuada.

\subsection{The construction}

Let $\mathscr{D}_{1}$ and $\mathscr{D}_{2}$ be two small DG-categories. Consider a bimodule $\varphi \in\left(\mathscr{D}_{2}^{\mathrm{op}} \otimes \mathscr{D}_{1}\right)$-dgm. The DG-category $\mathscr{D}_{1} \times_{\varphi} \mathscr{D}_{2}$, called the gluing of $\mathscr{D}_{1}$ with $\mathscr{D}_{2}$ along $\varphi$, is defined as follows:

(1) The objects of the DG-category $\mathscr{D}_{1} \times_{\varphi} \mathscr{D}_{2}$ are triples $M=\left(M_{1}, M_{2}, \mu\right)$, where $M_{i} \in \mathscr{D}_{i}$ and $\mu \in \varphi\left(M_{2}, M_{1}\right)$ is a closed element of degree 0 (recall that being a bimodule $\varphi$ associates a complex $\varphi\left(M_{2}, M_{1}\right)$ to any pair $\left(M_{1}, M_{2}\right)$ of objects of $\mathscr{D}_{1}$ and $\mathscr{D}_{2}$ ).

(2) The morphism complexes are defined to be the sums

$$
\operatorname{Hom}_{\mathscr{D}_{1} \times_{\varphi} \mathscr{D}_{2}}^{k}(M, N)=\operatorname{Hom}_{\mathscr{D}_{1}}^{k}\left(M_{1}, N_{1}\right) \oplus \operatorname{Hom}_{\mathscr{D}_{2}}^{k}\left(M_{2}, N_{2}\right) \oplus \varphi^{k-1}\left(N_{2}, M_{1}\right)
$$

with the differentials given by

$$
d\left(f_{1}, f_{2}, f_{21}\right)=\left(d\left(f_{1}\right), d\left(f_{2}\right),-d\left(f_{21}\right)-f_{2} \circ \mu+v \circ f_{1}\right)
$$

where $M=\left(M_{1}, M_{2}, \mu\right)$ and $N=\left(N_{1}, N_{2}, \nu\right)$.

(3) The multiplication is defined by

$$
\left(f_{1}, f_{2}, f_{21}\right) \circ\left(g_{1}, g_{2}, g_{21}\right)=\left(f_{1} \circ g_{1}, f_{2} \circ g_{2}, f_{21} \circ g_{1}+(-1)^{\operatorname{deg} f_{2}} f_{2} \circ g_{21}\right),
$$

where $f \in \operatorname{Hom}_{\mathscr{D}_{1} \times_{\varphi} \mathscr{D}_{2}}(M, N)$ and $g \in \operatorname{Hom}_{\mathscr{D}_{1} \times_{\varphi} \mathscr{D}_{2}}(L, M)$. In particular, the identity morphism of $\left(M_{1}, M_{2}, \mu\right)$ is given by

$$
\operatorname{id}_{\left(M_{1}, M_{2}, \mu\right)}=\left(\operatorname{id}_{M_{1}}, \operatorname{id}_{M_{2}}, 0\right) .
$$

It is an exercise left to the reader to check that this is a DG-category.

Remark 4.1. Another way to understand the definition of the gluing category is by saying that there is a distinguished triangle

$$
\operatorname{Hom}_{\mathscr{D}_{1} \times_{\varphi} \mathscr{D}_{2}}(M, N) \rightarrow \operatorname{Hom}_{\mathscr{D}_{1}}\left(M_{1}, N_{1}\right) \oplus \operatorname{Hom}_{\mathscr{D}_{2}}\left(M_{2}, N_{2}\right) \stackrel{(-v, \mu)}{\longrightarrow} \varphi\left(N_{2}, M_{1}\right)
$$

of complexes of vector spaces (the morphism is given by $\left(f_{1}, f_{2}\right) \mapsto f_{2} \circ \mu-v \circ f_{1}$ ). 
Remark 4.2. The upper triangular DG-category corresponding to DG-categories $\mathscr{D}_{1}$ and $\mathscr{D}_{2}$ and a bimodule $\varphi$ defined in [25] by Tabuada is DG-equivalent to the full DGsubcategory $\mathscr{D}_{1} \sqcup_{\varphi} \mathscr{D}_{2} \subset \mathscr{D}_{1} \times_{\varphi[1]} \mathscr{D}_{2}$ with objects of the form $\left(M_{1}, 0,0\right)$ and $\left(0, M_{2}, 0\right)$ only. One can show that the derived category of $\mathscr{D}_{1} \sqcup_{\varphi} \mathscr{D}_{2}$ is equivalent to the derived category of $\mathscr{D}_{1} \times_{\varphi} \mathscr{D}_{2}$, and moreover, even pretriangulated envelopes of these DG-categories coincide. The advantage of the DG-category $\mathscr{D}_{1} \sqcup_{\varphi} \mathscr{D}_{2}$ is that it is smaller and its definition is simpler. The advantage of $\mathscr{D}_{1} \times_{\varphi} \mathscr{D}_{2}$ is that it is more close to a pretriangulated category as is shown in the following lemma.

Lemma 4.3. Assume that the categories $\mathscr{D}_{1}$ and $\mathscr{D}_{2}$ are pretriangulated. Then, so is the gluing category $\mathscr{D}_{1} \times_{\varphi} \mathscr{D}_{2}$.

Proof. We must check that the shift of an object and the cone of a morphism are representable in $\mathscr{D}_{1} \times_{\varphi} \mathscr{D}_{2}$. For the shift this is evident-it is clear that the shift of the object $\left(M_{1}, M_{2}, \mu\right)$ is represented by the object $\left(M_{1}[1], M_{2}[1], \mu\right)$. To check representability of the cone, choose a closed morphism of degree zero from $\left(M_{1}, M_{2}, \mu\right)$ to $\left(N_{1}, N_{2}, v\right)$. By definition it is given by a pair of closed degree zero morphisms $f_{1}: M_{1} \rightarrow N_{1}$ and $f_{2}: M_{2} \rightarrow N_{2}$ and an element $f_{21} \in \varphi\left(M_{2}, N_{1}\right)$ of degree -1 such that

$$
d\left(f_{21}\right)=v \circ f_{1}-f_{2} \circ \mu
$$

(thus, $f_{21}$ is a homotopy between $f_{2} \circ \mu$ and $v \circ f_{1}$ ). Let $C_{1}$ be the cone of $f_{1}$ and let $C_{2}$ be the cone of $f_{2}$. As was observed in Remark 3.1, the cones come with degree zero morphisms

$$
M_{k}[1] \stackrel{i_{k}}{\rightarrow} C_{k} \stackrel{p_{k}}{\rightarrow} M_{k}[1], \quad N_{k} \stackrel{j_{k}}{\rightarrow} C_{k} \stackrel{s_{k}}{\rightarrow} N_{k}
$$

which give decompositions $C_{k}=M_{k}[1] \oplus N_{k}$ in the graded categories associated with $\mathscr{D}_{k}$. Moreover, we have

$$
d\left(j_{k}\right)=d\left(p_{k}\right)=0, \quad d\left(i_{k}\right)=j_{k} f_{k}, \quad d\left(s_{k}\right)=-f_{k} p_{k}
$$

Now, we consider the element

$$
\gamma=i_{2} \mu p_{1}+j_{2} \nu s_{1}+j_{2} f_{21} p_{1} \in \varphi\left(C_{2}, C_{1}\right) .
$$


The triple $\left(C_{1}, C_{2}, \gamma\right)$ is then an object of $\mathscr{D}_{1} \times_{\varphi} \mathscr{D}_{2}$. Indeed,

$$
\begin{aligned}
d \gamma & =\left(d i_{2}\right) \mu p_{1}+j_{2} v\left(d s_{1}\right)+j_{2}\left(d f_{21}\right) p_{1} \\
& =j_{2} f_{2} \mu p_{1}-j_{2} v f_{1} p_{1}+j_{2}\left(d f_{21}\right) p_{1}=j_{2}\left(f_{2} \mu-v f_{1}+d f_{21}\right) p_{1}=0
\end{aligned}
$$

so $\gamma$ is a closed element of degree 0 . Using again Remark 3.1, it is straightforward to check that $\left(C_{1}, C_{2}, \gamma\right)$ is the cone of the morphism we have started with-indeed, one can take $i=\left(i_{1}, i_{2}, 0\right), p=\left(p_{1}, p_{2}, 0\right), j=\left(j_{1}, j_{2}, 0\right)$, and $s=\left(s_{1}, s_{2}, 0\right)$ and check that all the required relations hold.

\subsection{Semiorthogonal decomposition}

For brevity, we denote by $\mathscr{D}$ the gluing category $\mathscr{D}_{1} \times_{\varphi} \mathscr{D}_{2}$. Our next goal is to describe the relation of DG-category $\mathscr{D}$ with the original categories $\mathscr{D}_{1}$ and $\mathscr{D}_{2}$. We start by introducing some natural DG-functors:

$$
\begin{array}{ll}
i_{1}: \mathscr{D}_{1} \rightarrow \mathscr{D}, \quad M_{1} \mapsto\left(M_{1}, 0,0\right), \\
i_{2}: \mathscr{D}_{2} \rightarrow \mathscr{D}, \quad M_{2} \mapsto\left(0, M_{2}, 0\right) .
\end{array}
$$

It turns out that these functors have adjoints on the DG-level.

\section{Lemma 4.4.}

(i) The left and the right adjoints of $i_{1}$ and $i_{2}$, respectively, are given by

$$
\begin{aligned}
& i_{1}^{*}: \mathscr{D} \rightarrow \mathscr{D}_{1}, \quad\left(M_{1}, M_{2}, \mu\right) \mapsto M_{1}, \\
& i_{2}^{!}: \mathscr{D} \rightarrow \mathscr{D}_{2}, \quad\left(M_{1}, M_{2}, \mu\right) \mapsto M_{2} .
\end{aligned}
$$

(ii) Assume that $\mathscr{D}_{1}$ is pretriangulated. The right adjoint of $i_{1}$ is given by

$$
i_{1}^{!}: \mathscr{D} \rightarrow \mathscr{D}_{1}-\mathrm{dgm}, \quad\left(N_{1}, N_{2}, v\right) \mapsto \operatorname{Cone}(v)[-1] .
$$

Here, we use a natural identification $\varphi\left(N_{2}, N_{1}\right)=\operatorname{Hom}_{\mathscr{D}_{1}-\operatorname{dgm}}\left(\mathrm{Y}^{N_{1}}, \varphi\left(N_{2},-\right)\right)$, so $v$ is considered as a closed morphism $Y^{N_{1}} \rightarrow \varphi\left(N_{2},-\right)$, and so we can speak about its cone. 
Proof. (i) Indeed, recalling the definition of $\mathscr{D}$, we see that

$$
\operatorname{Hom}_{\mathscr{D}}\left(\left(N_{1}, N_{2}, v\right), i_{1}\left(M_{1}\right)\right)=\operatorname{Hom}_{\mathscr{D}}\left(\left(N_{1}, N_{2}, v\right),\left(M_{1}, 0,0\right)\right)=\operatorname{Hom}_{\mathscr{D}_{1}}\left(N_{1}, M_{1}\right),
$$

hence $i_{1}^{*}\left(N_{1}, N_{2}, v\right)=N_{1}$. Analogously,

$$
\operatorname{Hom}_{\mathscr{D}}\left(i_{2}\left(M_{2}\right),\left(N_{1}, N_{2}, v\right)\right)=\operatorname{Hom}_{\mathscr{D}}\left(\left(0, M_{2}, 0\right),\left(N_{1}, N_{2}, v\right)\right)=\operatorname{Hom}_{\mathscr{D}_{2}}\left(M_{2}, N_{2}\right),
$$

hence $i_{2}^{!}\left(N_{1}, N_{2}, v\right)=N_{2}$.

(ii) We have

$$
\begin{aligned}
\operatorname{Hom}_{\mathscr{D}}\left(i_{1}\left(M_{1}\right),\left(N_{1}, N_{2}, v\right)\right) & =\operatorname{Hom}_{\mathscr{D}}\left(\left(M_{1}, 0,0\right),\left(N_{1}, N_{2}, v\right)\right) \\
& =\operatorname{Hom}_{\mathscr{D}_{1}}\left(M_{1}, N_{1}\right) \oplus \varphi\left(N_{2}, M_{1}\right)[-1] .
\end{aligned}
$$

If we think of $\varphi\left(N_{2}, M_{1}\right)$ as of the Hom-complex from $Y^{M_{1}}$ to $\varphi\left(N_{2},-\right)$ in the category of $\mathscr{D}_{1}$-modules, then the differential of the RHS will match with that of the Hom-complex from $Y^{M_{1}}$ to the shifted by -1 cone of $v$ considered as a morphism from $Y^{N_{1}}$ to $\varphi\left(N_{2},-\right)$. This shows that $i_{1}^{!}(N)=\operatorname{Cone}(v)[-1]$.

Using formulas (21), (22), and (23), one easily computes

$$
i_{1}^{*} \circ i_{1}=\mathrm{id}_{\mathscr{D}_{1}}, \quad i_{2}^{!} \circ i_{2}=\mathrm{id}_{\mathscr{D}_{2}}, \quad i_{1}^{*} \circ i_{2}=0, \quad i_{1}^{!} \circ i_{2}=\varphi[-1]
$$

Recall that the last equation agrees with the definition of the gluing functor for semiorthogonal decompositions discussed in Section 2.2. In fact, we have the following corollary.

Corollary 4.5. Assume that $\mathscr{D}_{1}$ and $\mathscr{D}_{2}$ are small pretriangulated categories (hence so is $\mathscr{D})$. The functors $i_{1}:\left[\mathscr{D}_{1}\right] \rightarrow[\mathscr{D}]$ and $i_{2}:\left[\mathscr{D}_{2}\right] \rightarrow[\mathscr{D}]$ are fully faithful and give a semiorthogonal decomposition $[\mathscr{D}]=\left\langle\left[\mathscr{D}_{1}\right],\left[\mathscr{D}_{2}\right]\right\rangle$ with the gluing bifunctor induced by $\varphi$.

Proof. We define $\mathscr{T}_{i}=\left[\mathscr{D}_{i}\right], \mathscr{T}=[\mathscr{D}]$ for short. The DG-functors $i_{1}, i_{2}, i_{1}^{*}$, and $i_{2}^{!}$descend to triangulated functors between the homotopy categories; moreover, the adjunctions are preserved. So, the first two equations of (24) prove that $i_{1}: \mathscr{T}_{1} \rightarrow \mathscr{T}$ and $i_{2}: \mathscr{T}_{2} \rightarrow \mathscr{T}$ are fully faithful and have left and right adjoints, respectively. The third equation shows that the essential images of $\mathscr{T}_{1}$ and $\mathscr{T}_{2}$ in $\mathscr{T}$ are semiorthogonal. Moreover, the functor 
$i_{1}^{!} \circ i_{2}[1]: \mathscr{T}_{2} \rightarrow \mathscr{T}_{1}$ is isomorphic to the functor $L \varphi$ induced by the bimodule $\varphi$ by the fourth equation. So, it remains to check that the subcategories $\mathscr{T}_{1}$ and $\mathscr{T}_{2}$ generate $\mathscr{T}$. Indeed, by Lemma 2.2 there is a semiorthogonal decomposition $\mathscr{T}=\left\langle\mathscr{T}_{1},{ }^{\perp} \mathscr{T}_{1} \cap \mathscr{T}_{2}{ }^{\perp}, \mathscr{T}_{2}\right\rangle$, where

$$
{ }^{\perp} \mathscr{T}_{1} \cap \mathscr{T}_{2}^{\perp}=\operatorname{Ker} i_{1}^{*} \cap \operatorname{Ker} i_{2}^{!},
$$

so it remains to check that if both $i_{1}^{*} M=M_{1}$ and $i_{2}^{!} M=M_{2}$ are null-homotopic, then $M$ is null-homotopic as well. Indeed, let $h_{1} \in \operatorname{Hom}_{\mathscr{D}_{1}}^{-1}\left(M_{1}, M_{1}\right)$ and $h_{2} \in \operatorname{Hom}_{\mathscr{D}_{2}}^{-1}\left(M_{2}, M_{2}\right)$ be the contracting homotopies of $\mathrm{id}_{M_{1}}$ and $\mathrm{id}_{M_{2}}$, respectively. In other words, we assume that $d_{1}\left(h_{1}\right)=\mathrm{id}_{M_{1}}$ and $d_{2}\left(h_{2}\right)=\mathrm{id}_{M_{2}}$. We want to extend it to a homotopy of $\mathrm{id}_{M}$. This means that we have to find $h_{21} \in \varphi\left(M_{2}, M_{1}\right)$ such that

$$
d\left(h_{21}\right)=h_{2} \circ \mu-\mu \circ h_{1}
$$

For this, we first note that the RHS above is closed. Indeed, since $d \mu=0$, we have

$$
d\left(h_{2} \circ \mu-\mu \circ h_{1}\right)=d\left(h_{2}\right) \circ \mu-\mu \circ d\left(h_{1}\right)=\mu-\mu=0 .
$$

Let us take

$$
h_{21}=\left(h_{2} \circ \mu-\mu \circ h_{1}\right) \circ h_{1} .
$$

Since the expression in parentheses is a closed morphism, we have

$$
d\left(h_{21}\right)=\left(h_{2} \circ \mu-\mu \circ h_{1}\right) \circ d\left(h_{1}\right)=h_{2} \circ \mu-\mu \circ h_{1},
$$

and we are done.

\subsection{Derived category}

One has also a semiorthogonal decomposition of the derived category of the gluing. First, note that by Proposition 3.9 the functors $i_{1}$ and $i_{2}$ defined by (21) extend to derived categories and give functors that we denote here by

$$
I_{1}=\operatorname{LInd}_{i_{1}}: \mathbf{D}\left(\mathscr{D}_{1}\right) \rightarrow \mathbf{D}(\mathscr{D}), \quad I_{2}=\operatorname{LInd}_{i_{2}}: \mathbf{D}\left(\mathscr{D}_{2}\right) \rightarrow \mathbf{D}(\mathscr{D})
$$

and that have right adjoints denoted here by

$$
I_{1}^{!}=\operatorname{Res}_{i_{1}}: \mathbf{D}(\mathscr{D}) \rightarrow \mathbf{D}\left(\mathscr{D}_{1}\right), \quad I_{2}^{!}=\operatorname{Res}_{i_{2}}: \mathbf{D}(\mathscr{D}) \rightarrow \mathbf{D}\left(\mathscr{D}_{2}\right),
$$


induced by restriction of DG-modules. In other words,

$$
I_{1}^{!}(F)=F_{i_{1}}, \quad I_{2}^{!}(F)=F_{i_{2}},
$$

for any $F \in \mathscr{D}$-dgm.

Proposition 4.6. Let $\mathscr{D}=\mathscr{D}_{1} \times{ }_{\varphi} \mathscr{D}_{2}$. Then, one has a semiorthogonal decomposition

$$
\mathbf{D}(\mathscr{D})=\left\langle\mathbf{D}\left(\mathscr{D}_{1}\right), \mathbf{D}\left(\mathscr{D}_{2}\right)\right\rangle
$$

with the gluing functor isomorphic to $L \varphi: \mathbf{D}\left(\mathscr{D}_{2}\right) \rightarrow \mathbf{D}\left(\mathscr{D}_{1}\right)$. Moreover, for any DG-module $F$ over $\mathscr{D}$ there is a distinguished triangle

$$
F_{i_{1}} \rightarrow I_{1}^{*} F \rightarrow F_{i_{2}} \stackrel{\mathbb{L}}{\otimes} \mathscr{D}_{2} \varphi
$$

Note that one does not need to assume that any of the categories is pretriangulated.

Proof. By Proposition 3.9, the functors $I_{1}$ and $I_{2}$ are fully faithful. Moreover,

$$
I_{2}^{!} I_{1}(F)=\left(F \stackrel{\mathbb{L}}{\otimes} \mathscr{D}_{1} i_{1} \mathscr{D}\right)_{i_{2}}=F \stackrel{\mathbb{L}}{\otimes} \mathscr{D}_{1} i_{1} \mathscr{D}_{i_{2}}=0
$$

since $_{i_{1}} \mathscr{D}_{i_{2}}=0$ by the definition of gluing. Applying two times Lemma 2.2, we conclude that there is a semiorthogonal decomposition $\mathbf{D}(\mathscr{D})=\left\langle\mathscr{T}, \mathbf{D}\left(\mathscr{D}_{1}\right), \mathbf{D}\left(\mathscr{D}_{2}\right)\right\rangle$, where

$$
\mathscr{T}=\mathbf{D}\left(\mathscr{D}_{1}\right)^{\perp} \cap \mathbf{D}\left(\mathscr{D}_{2}\right)^{\perp}
$$

It remains to check that $\mathscr{T}=0$. For this, we note that, for any object $\left(M_{1}, M_{2}, \mu\right)$ of $\mathscr{D}$, one can consider $\mu \in \varphi\left(M_{2}, M_{1}\right)$ as a morphism from $Y^{\left(M_{1}, 0,0\right)}$ to $Y^{\left(0, M_{2}, 0\right)}[1]$ in $\mathbf{D}(\mathscr{D})$, and the cone of this morphism is a representable $\mathscr{D}$-module, represented precisely by the initial object $\left(M_{1}, M_{2}, \mu\right)$. In other words, in $\mathbf{D}(\mathscr{D})$ there is a canonical distinguished triangle

$$
Y^{\left(0, M_{2}, 0\right)} \longrightarrow Y^{\left(M_{1}, M_{2}, \mu\right)} \longrightarrow Y^{\left(M_{1}, 0,0\right)} \stackrel{\mu}{\longrightarrow} Y^{\left(0, M_{2}, 0\right)}[1]
$$

In particular, it follows that all representable $\mathscr{D}$-modules are contained in the subcategory of $\mathbf{D}(\mathscr{D})$ generated by $\mathbf{D}\left(\mathscr{D}_{1}\right)$ and $\mathbf{D}\left(\mathscr{D}_{2}\right)$. Thus, $\mathscr{T}$ is orthogonal to all representable $\mathscr{D}$-modules, hence $\mathscr{T}=0$. 
To establish the formula for the gluing functor $\phi: \mathbf{D}\left(\mathscr{D}_{2}\right) \rightarrow \mathbf{D}\left(\mathscr{D}_{1}\right)$, we recall that, as mentioned in Section 2.2, we have $\phi=I_{1}^{!} I_{2}[1]$. By definition of $I_{1}^{!}$and $I_{2}$, we have

$$
I_{1}^{!}\left(I_{2}(N)\right)=\left(N \stackrel{\mathbb{L}}{\otimes} \mathscr{D}_{2} i_{2} \mathscr{D}\right)_{i_{1}}=N \stackrel{\mathbb{L}}{\otimes} \mathscr{D}_{2} i_{2} \mathscr{D}_{i_{1}}
$$

and it remains to note that, by (18), we have $i_{2} \mathscr{D}_{i_{1}}=\varphi[-1]$, hence $\phi(N) \cong N \stackrel{\mathbb{L}}{\otimes} \mathscr{D}_{2} \varphi$.

Finally, to check (26), we first consider the standard triangle

$$
I_{2} I_{2}^{!} F \rightarrow F \rightarrow I_{1} I_{1}^{*} F
$$

Applying the functor $I_{1}^{!}$, we obtain

$$
I_{1}^{!} I_{2} I_{2}^{!} F \rightarrow I_{1}^{!} F \rightarrow I_{1}^{*} F
$$

since $I_{1}^{!} \circ I_{1}$ is the identity functor. As we already checked that $I_{1}^{!} I_{2}$ is isomorphic to the derived tensor product by $\varphi[-1]$, using (25) we deduce the claim.

\subsection{Smoothness and properness}

It is useful to note that the quasiequivalence class of the gluing depends only on the quasiisomorphism class of the gluing bimodule.

Lemma 4.7. Assume that $\varphi$ and $\psi$ are quasiisomorphic $\left(\mathscr{D}_{2}^{\mathrm{op}} \otimes \mathscr{D}_{1}\right)$-modules. Then, the gluings $\mathscr{D}_{1} \times_{\varphi} \mathscr{D}_{2}$ and $\mathscr{D}_{1} \times_{\psi} \mathscr{D}_{2}$ are quasiequivalent.

Proof. First assume that $\xi: \varphi \rightarrow \psi$ is a closed degree 0 morphism in the category of bimodules which is a quasiisomorphism. Then, we define a DG-functor from $\mathscr{D}_{1} \times_{\varphi} \mathscr{D}_{2}$ to $\mathscr{D}_{1} \times_{\psi} \mathscr{D}_{2}$ by

$$
\left(M_{1}, M_{2}, \mu\right) \mapsto\left(M_{1}, M_{2}, \xi(\mu)\right)
$$

on objects and by

$$
\left(f_{1}, f_{2}, f_{21}\right) \mapsto\left(f_{1}, f_{2}, \xi\left(f_{21}\right)\right)
$$

on morphisms. It is very easy to check that it is a DG-functor that acts by quasiisomorphisms on Hom complexes. To check that it is a quasiequivalence, we have to show that it is homotopically essentially surjective. In other words, for any $M=\left(M_{1}, M_{2}, v\right) \in$ $\mathscr{D}_{1} \times_{\psi} \mathscr{D}_{2}$ we have to construct an object in $\mathscr{D}_{1} \times_{\varphi} \mathscr{D}_{2}$ whose image is homotopy equivalent to $M$. For this, we choose $\mu \in \varphi\left(M_{2}, M_{1}\right)$ such that $\xi(\mu)$ is homologous to $v$ and take $\left(M_{1}, M_{2}, \mu\right)$. So, we have to show that $\left(M_{1}, M_{2}, \xi(\mu)\right)$ is homotopic to $\left(M_{1}, M_{2}, \nu\right)$. Since 
$\xi(\mu)$ and $v$ are homologous, we have $v-\xi(\mu)=d \rho$ for some $\rho \in \psi\left(M_{2}, M_{1}\right)[-1]$. Therefore, $\left(\mathrm{id}_{M_{1}}, \mathrm{id}_{M_{2}}, \rho\right)$ gives a morphism from $\left(M_{1}, M_{2}, \xi(\mu)\right)$ to $\left(M_{1}, M_{2}, v\right)$ and $\left(\mathrm{id}_{M_{1}}, \mathrm{id}_{M_{2}},-\rho\right)$ gives a morphism in the opposite direction. The compositions are the identity morphisms, and so we are done.

Thus, we have proved the result when the quasiisomorphism is given by a morphism. In the general case, we can connect $\varphi$ to $\psi$ by a chain of quasiisomorphisms; each of them gives a quasiequivalence. Thus, the gluings are connected by a chain of quasiequivalences, so the categories are quasiequivalent.

For many purposes, it is convenient to have an h-projective gluing bimodule. By the above lemma, we can always replace $\varphi$ with its h-projective resolution without changing the quasiequivalence class of the gluing DG-category.

Remark 4.8. Further, we will also show that replacing the categories $\mathscr{D}_{1}$ and $\mathscr{D}_{2}$ by quasiequivalent DG-categories, one does not change the quasiequivalence class of the gluing; see Proposition 4.14.

Recall the notion of smoothness and properness of a DG-category; see Section 3.7.

Proposition 4.9. Let $\mathscr{D}_{1}$ and $\mathscr{D}_{2}$ be smooth DG-categories. A gluing $\mathscr{D}=\mathscr{D}_{1} \times_{\varphi} \mathscr{D}_{2}$ is smooth if the gluing bimodule $\varphi$ is perfect in $\mathbf{D}\left(\mathscr{D}_{2}^{\mathrm{op}} \otimes \mathscr{D}_{1}\right)$. If $\mathscr{D}_{1}$ and $\mathscr{D}_{2}$ are proper and $\varphi$ is perfect, then $\mathscr{D}$ is proper.

Proof. Our goal is to show that the diagonal bimodule $\mathscr{D}$ is perfect. Using the same argument as in [17, Proposition 3.11], we conclude that $\mathscr{D}$ fits into a distinguished triangle

$$
\mathscr{D}_{1} \stackrel{\mathbb{L}}{\otimes}_{\mathscr{D}_{1}^{\mathrm{op}} \otimes \mathscr{D}_{1}}\left(\mathscr{D}^{\mathrm{op}} \otimes \mathscr{D}\right) \oplus \mathscr{D}_{2} \stackrel{\mathbb{L}}{\otimes}_{\mathscr{D}_{2}^{\mathrm{op}} \otimes \mathscr{D}_{2}}\left(\mathscr{D}^{\mathrm{op}} \otimes \mathscr{D}\right) \rightarrow \mathscr{D} \rightarrow \varphi \stackrel{\mathbb{L}}{\otimes}_{\mathscr{D}_{2}^{\mathrm{op}} \otimes \mathscr{D}_{1}}\left(\mathscr{D}^{\mathrm{op}} \otimes \mathscr{D}\right)
$$

and observe that in the first term both summands are perfect since $\mathscr{D}_{i}$ is a perfect bimodule over $\mathscr{D}_{i}$ by the smoothness of $\mathscr{D}_{i}$. Thus, $\mathscr{D}$ is perfect if the third term is perfect. It remains to note that perfectness of $\varphi$ in $\mathbf{D}\left(\mathscr{D}_{2}^{\mathrm{op}} \otimes \mathscr{D}_{1}\right)$ implies perfectness of $\varphi \stackrel{\mathbb{L}}{\otimes}_{\mathscr{D}_{2}^{\mathrm{op}} \otimes \mathscr{D}_{1}}\left(\mathscr{D}^{\mathrm{op}} \otimes \mathscr{D}\right)$ in $\mathbf{D}\left(\mathscr{D}^{\mathrm{op}} \otimes \mathscr{D}\right)$ by extension of scalars.

The properness of $\mathscr{D}$ follows immediately from the definition. 


\subsection{The gluing and semiorthogonal decompositions}

Here, we show that any category with a semiorthogonal decomposition is quasiequivalent to a gluing.

Proposition 4.10. Assume that $\mathscr{D}$ is a pretriangulated category and a semiorthogonal decomposition of its homotopy category is given:

$$
[\mathscr{D}]=\left\langle\mathscr{T}_{1}, \mathscr{T}_{2}\right\rangle
$$

Then, DG-category $\mathscr{D}$ is quasiequivalent to a gluing of two pretriangulated DGcategories $\mathscr{D}_{1}$ and $\mathscr{D}_{2}$ such that $\left[\mathscr{D}_{1}\right]=\mathscr{T}_{1}$ and $\left[\mathscr{D}_{2}\right]=\mathscr{T}_{2}$.

Proof. We take $\mathscr{D}_{k}$ to be the full DG-subcategory of $\mathscr{D}$ having the same objects as $\mathscr{T}_{k}$ and take

$$
\varphi:={ }_{i_{2}} \mathscr{D}_{i_{1}}[1]
$$

Note that both DG-categories $\mathscr{D}_{1}$ and $\mathscr{D}_{2}$ are pretriangulated. We construct the DGfunctor $\alpha: \mathscr{D}_{1} \times_{\varphi} \mathscr{D}_{2} \rightarrow \mathscr{D}$-dgm as follows. On objects it is defined by

$$
\alpha\left(M_{1}, M_{2}, \mu\right)=\text { Cone }\left(\mu: M_{1}[-1] \rightarrow M_{2}\right)
$$

Here, we observe that $\mu \in \varphi\left(M_{2}, M_{1}\right)=\operatorname{Hom}_{\mathscr{D}}\left(M_{1}, M_{2}\right)[1]=\operatorname{Hom}_{\mathscr{D}}\left(M_{1}[-1], M_{2}\right)$ by definition of $\varphi$. Note that if $\mu: M_{1}[-1] \rightarrow M_{2}$ and $v: N_{1}[-1] \rightarrow N_{2}$ are two closed morphisms of degree zero, then the cones $C_{M}=\operatorname{Cone}\left(\mu: M_{1}[-1] \rightarrow M_{2}\right)$ and $C_{N}=$ Cone $\left(v: N_{1}[-1] \rightarrow N_{2}\right)$ come with direct sum decompositions

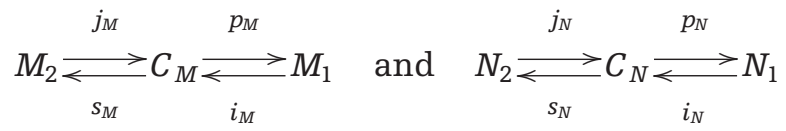

with

$$
\begin{aligned}
& d j_{M}=d p_{M}=0, \quad d i_{M}=j_{M} \mu, \quad d s_{M}=-\mu p_{M}, \\
& d j_{N}=d p_{N}=0, \quad d i_{N}=j_{N} v, \quad d s_{N}=-v p_{N} .
\end{aligned}
$$

It follows that any $f \in \operatorname{Hom}_{\mathscr{D}}\left(C_{M}, C_{N}\right)$ can be written as

$$
f=\left(i_{N} p_{N}+j_{N} s_{N}\right) f\left(i_{M} p_{M}+j_{M} s_{M}\right)=i_{N} f_{11} p_{M}+i_{N} f_{12} s_{M}+j_{N} f_{21} p_{M}+j_{N} f_{22} s_{M},
$$


where

$$
\begin{array}{ll}
f_{11}=p_{N} f i_{M} \in \operatorname{Hom}_{\mathscr{D}}\left(M_{1}, N_{1}\right), & f_{12}=p_{N} f j_{M} \in \operatorname{Hom}_{\mathscr{D}}\left(M_{2}, N_{1}\right), \\
f_{21}=s_{N} f i_{M} \in \operatorname{Hom}_{\mathscr{D}}\left(M_{1}, N_{2}\right), & f_{22}=s_{N} f j_{M} \in \operatorname{Hom}_{\mathscr{D}}\left(M_{2}, N_{2}\right) .
\end{array}
$$

Therefore,

$$
\begin{aligned}
d f= & d\left(i_{N} f_{11} p_{M}+i_{N} f_{12} s_{M}+j_{N} f_{21} p_{M}+j_{N} f_{22} s_{M}\right) \\
= & i_{N}\left(d f_{11}-(-1)^{\operatorname{deg} f} f_{12} \mu\right) p_{M}+i_{N} d f_{12} s_{M} \\
& +j_{N}\left(d f_{21}+v f_{11}-(-1)^{\operatorname{deg} f} f_{22} \mu\right) p_{M}+j_{N}\left(d f_{22}+v f_{12}\right) s_{M} .
\end{aligned}
$$

This formula shows that the map

$$
\operatorname{Hom}_{\mathscr{D}}\left(C_{M}, C_{N}\right) \rightarrow \operatorname{Hom}_{\mathscr{D}}\left(M_{2}, N_{1}\right), \quad f \mapsto f_{12}
$$

is a morphism of complexes. Moreover, the complex $\operatorname{Hom}_{\mathscr{D}}\left(M_{2}, N_{1}\right)$ is acyclic, as the cohomology of $\operatorname{Hom}_{\mathscr{D}}\left(M_{2}, N_{1}\right)$ is just Ext spaces from $M_{2}$ to $N_{1}$ that are zero since $M_{2} \in \mathscr{T}_{2}$ and $N_{1} \in \mathscr{T}_{1}$ and those subcategories of $\mathscr{T}$ are semiorthogonal. It follows that the map

$$
\begin{aligned}
& \alpha: \operatorname{Hom}_{\mathscr{D}_{1}}\left(M_{1}, N_{1}\right) \oplus \operatorname{Hom}_{\mathscr{D}_{2}}\left(M_{2}, N_{2}\right) \oplus \operatorname{Hom}_{\mathscr{D}}\left(M_{1}, N_{2}\right) \rightarrow \operatorname{Hom}_{\mathscr{D}}\left(C_{M}, C_{N}\right), \\
& \left(f_{11}, f_{22}, f_{21}\right) \mapsto i_{N} f_{11} p_{M}+j_{N} f_{21} p_{M}+j_{N} f_{22} s_{M}
\end{aligned}
$$

is a quasiisomorphism. But since $\operatorname{Hom}_{\mathscr{D}}\left(M_{1}, N_{2}\right)=\mathscr{D}\left(N_{2}, M_{1}\right)=\varphi\left(N_{2}, M_{1}\right)[-1]$ (and so the differentials of $\mathscr{D}$ and $\varphi$ differ by a sign), it follows that the LHS literally coincides with $\operatorname{Hom}_{\mathscr{D}_{1} \times \varphi \mathscr{D}_{2}}\left(\left(M_{1}, M_{2}, \mu\right),\left(N_{1}, N_{2}, v\right)\right)$ and we can consider the above map as a definition of the functor $\alpha$ on morphisms. One can easily check that this is compatible with the compositions, and so correctly defines a DG-functor. And since we just checked that the morphism on Hom-complexes is a quasiisomorphism, the functor $\alpha$ is quasi-fully faithful.

To show that it is a quasiequivalence, we have to check that any object of $\mathscr{D}$ is homotopic to an object in the image of $\alpha$. Take any $M$ in $\mathscr{D}$ and let

$$
M_{1}[-1] \rightarrow M_{2} \rightarrow M \rightarrow M_{1}
$$

be its decomposition in $\mathscr{T}$ with respect to the original semiorthogonal decomposition. Let $\mu: M_{1}[-1] \rightarrow M_{2}$ be the morphism from that triangle. Then $\alpha\left(M_{1}, M_{2}, \mu\right)=$ Cone $(\mu)$, 
which is isomorphic to $M$ in $\mathscr{T}=[\mathscr{D}]$. Thus, in $\mathscr{D}$ there is a homotopy equivalence of $M$ and $\alpha\left(M_{1}, M_{2}, \mu\right)$.

\subsection{Regluing}

Recall the notion of a partial categorical DG-resolution 1.5. Assume that $\mathscr{D}_{1}, \mathscr{D}_{2}$ are small pretriangulated DG-categories and $\varphi \in\left(\mathscr{D}_{2}^{\mathrm{op}} \otimes \mathscr{D}_{1}\right)$-dgm is a DG-bimodule. Assume also that $\tau_{1}: \mathscr{D}_{1} \rightarrow \tilde{\mathscr{D}}_{1}$ and $\tau_{2}: \mathscr{D}_{2} \rightarrow \tilde{\mathscr{D}}_{2}$ are partial categorical DG-resolutions and $\tilde{\varphi} \in\left(\tilde{\mathscr{D}}_{2}^{\text {op }} \otimes\right.$ $\tilde{\mathscr{D}}_{1}$ )-dgm is another DG-bimodule. We will say that the bimodules $\varphi$ and $\tilde{\varphi}$ are compatible if a quasiisomorphism

$$
C: \varphi \stackrel{\cong}{\tau_{2}} \tilde{\varphi}_{\tau_{1}}
$$

is given. Consider the gluings $\mathscr{D}=\mathscr{D}_{1} \times_{\varphi} \mathscr{D}_{2}$ and $\tilde{\mathscr{D}}=\tilde{\mathscr{D}}_{1} \times_{\tilde{\varphi}} \tilde{\mathscr{D}}_{2}$. We denote by $I_{1}, I_{2}$ the embedding functors of the semiorthogonal decomposition of $\mathbf{D}(\mathscr{D})$ and by $\tilde{I}_{1}, \tilde{I}_{2}$ the embedding functors of the semiorthogonal decomposition of $\mathbf{D}(\tilde{\mathscr{D}})$.

Proposition 4.11. If the bimodules $\varphi$ and $\tilde{\varphi}$ are compatible, then there is a DG-functor $\tau: \mathscr{D}_{1} \times_{\varphi} \mathscr{D}_{2} \rightarrow \tilde{\mathscr{D}}_{1} \times_{\tilde{\varphi}} \tilde{\mathscr{D}}_{2}$ which is a partial categorical DG-resolution, such that

(a) $\operatorname{Res} \tau_{1} \circ L \tilde{\varphi} \circ \operatorname{LInd}_{\tau_{2}} \cong L \varphi$, an isomorphism of functors $\mathbf{D}\left(\mathscr{D}_{2}\right) \rightarrow \mathbf{D}\left(\mathscr{D}_{1}\right)$;

(b) $\quad \tilde{I}_{k} \circ \operatorname{LInd}_{\tau_{k}} \cong \operatorname{LInd}_{\tau} \circ I_{k}$ for $k=1,2$, an isomorphism of functors $\mathbf{D}\left(\mathscr{D}_{k}\right) \rightarrow \mathbf{D}(\tilde{\mathscr{D}})$;

(c) $\operatorname{Res}_{\tau_{k}} \circ \tilde{I}_{k}^{!} \cong I_{k}^{!} \circ \operatorname{Res}_{\tau}$ for $k=1,2$, an isomorphism of functors $\mathbf{D}(\tilde{\mathscr{D}}) \rightarrow \mathbf{D}\left(\mathscr{D}_{k}\right)$;

(d) $\operatorname{Res}_{\tau} \circ \tilde{I}_{1} \cong I_{1} \circ \operatorname{Res}_{\tau_{1}}$, an isomorphism of functors $\mathbf{D}\left(\tilde{\mathscr{D}}_{1}\right) \rightarrow \mathbf{D}(\mathscr{D})$;

(e) if the canonical morphism $L \varphi \circ \operatorname{Res}_{\tau_{2}} \rightarrow \operatorname{Res} \tau_{1} \circ L \tilde{\varphi}$ of functors is an isomorphism, then we have $\operatorname{Res}_{\tau} \circ \tilde{I}_{2} \cong I_{2} \circ \operatorname{Res}_{\tau_{2}}$, an isomorphism of functors $\mathbf{D}\left(\tilde{\mathscr{D}}_{2}\right) \rightarrow \mathbf{D}(\mathscr{D})$.

Proof. First, we note that, for any $\left(M_{1}, M_{2}, \mu\right) \in \mathscr{D}_{1} \times_{\varphi} \mathscr{D}_{2}$, we have

$$
C(\mu) \in_{\tau_{2}} \tilde{\varphi}_{\tau_{1}}\left(M_{2}, M_{1}\right)=\tilde{\varphi}\left(\tau_{2}\left(M_{2}\right), \tau_{1}\left(M_{1}\right)\right),
$$

so $\left(\tau_{1}\left(M_{1}\right), \tau_{2}\left(M_{2}\right), C(\mu)\right)$ is an object of the gluing $\tilde{\mathscr{D}}_{1} \times_{\tilde{\varphi}} \tilde{\mathscr{D}}_{2}$. We define a DG-functor

$$
\tau: \mathscr{D}_{1} \times_{\varphi} \mathscr{D}_{2} \rightarrow \tilde{\mathscr{D}}_{1} \times_{\tilde{\varphi}} \tilde{\mathscr{D}}_{2}, \quad\left(M_{1}, M_{2}, \mu\right) \mapsto\left(\tau_{1}\left(M_{1}\right), \tau_{2}\left(M_{2}\right), c(\mu)\right)
$$

The fact that $\tau$ is a DG-functor is straightforward. Let us check that it is quasi-fully faithful. For this just note that $\operatorname{Hom}_{\tilde{D}}(\tau M, \tau N)$ equals to

$$
\operatorname{Hom}_{\tilde{\mathscr{D}}_{1}}\left(\tau_{1} M_{1}, \tau_{1} N_{1}\right) \oplus \operatorname{Hom}_{\tilde{\mathscr{D}}_{2}}\left(\tau_{2} M_{2}, \tau_{2} N_{2}\right) \oplus \tilde{\varphi}\left(\tau_{2} N_{2}, \tau_{1} M_{1}\right)[-1]
$$


with appropriate differential. The first summand here is quasiisomorphic to $\operatorname{Hom}_{\mathscr{D}_{1}}\left(M_{1}, N_{1}\right)$ since $\tau_{1}$ is quasi-fully faithful, the second summand is quasiisomorphic to $\operatorname{Hom}_{\mathscr{D}_{2}}\left(M_{2}, N_{2}\right)$ since $\tau_{2}$ is quasi-fully faithful, and the third summand is nothing but ${ }_{\tau_{2}} \tilde{\varphi}_{\tau_{1}}\left(N_{2}, M_{1}\right)[-1]$, which is quasiisomorphic via the morphism $c$ to $\varphi\left(N_{2}, M_{1}\right)[-1]$. Hence, $\tau$ is quasi-fully faithful.

It remains to verify properties (a)-(d). The first is clear:

$$
\operatorname{Res}_{\tau_{1}}\left(L \tilde{\varphi}\left(\operatorname{LInd}_{\tau_{2}}\left(M_{2}\right)\right)\right)=M_{2} \stackrel{\mathbb{L}}{\otimes} \mathscr{D}_{2} \tau_{2} \tilde{\mathscr{D}}_{2} \stackrel{\mathbb{L}}{\otimes} \tilde{\mathscr{D}}_{2} \tilde{\varphi}_{\tau_{1}} \cong M_{2} \stackrel{\mathbb{L}}{\otimes} \mathscr{D}_{2} \tau_{2} \tilde{\varphi}_{\tau_{1}} \cong M_{2} \stackrel{\mathbb{L}}{\otimes} \mathscr{D}_{2} \varphi=L \varphi\left(M_{2}\right),
$$

hence the required isomorphism of functors.

Let $\tilde{\imath}_{k}: \tilde{\mathscr{D}}_{k} \rightarrow \tilde{\mathscr{D}}$ be the embedding DG-functor. Then $\tilde{\imath}_{k} \circ \tau_{k}=\tau \circ i_{k}$ by definition of $\tau$. This implies an isomorphism of the induction functors $\operatorname{LInd}{d_{\tau_{k}}} \circ \operatorname{LInd}_{\tau_{k}} \cong \operatorname{LInd}_{\tau} \circ$ LInd $i_{i_{k}}$ which is precisely (b) by definition of $I_{k}$ and $\tilde{I}_{k}$. Note also that (c) follows from (b) by passing to the right adjoint functors.

Further, let us prove (d). First, consider the composition $I_{2}^{!} \circ \operatorname{Res}_{\tau} \circ \tilde{I}_{1}$. By (c), it is isomorphic to $\operatorname{Res}_{\tau_{2}} \circ \tilde{I}_{2}^{!} \circ \tilde{I}_{1}$. But $\tilde{I}_{2}^{!} \circ \tilde{I}_{1}=0$ by semiorthogonality of $\mathbf{D}\left(\tilde{\mathscr{D}}_{1}\right)$ and $\mathbf{D}\left(\tilde{\mathscr{D}}_{2}\right)$ in $\mathbf{D}(\tilde{\mathscr{D}})$. Thus we conclude that $I_{2}^{!} \circ \operatorname{Res}_{\tau} \circ \tilde{I}_{1}=0$. It follows from the semiorthogonal decomposition of $\mathbf{D}(\mathscr{D})$ that $\operatorname{Res}_{\tau} \circ \tilde{I}_{1}$ is in the image of $I_{1}$, hence

$$
\operatorname{Res}_{\tau} \circ \tilde{I}_{1}=I_{1} \circ I_{1}^{!} \circ \operatorname{Res}_{\tau} \circ \tilde{I}_{1} \cong I_{1} \circ \operatorname{Res}_{\tau_{1}} \circ \tilde{I}_{1}^{!} \circ \tilde{I}_{1} \cong I_{1} \circ \operatorname{Res}_{\tau_{1}}
$$

the first is since $I_{1}$ is fully faithful, the second is by (c), and the third is since $\tilde{I}_{1}$ is fully faithful.

Finally, let us prove (e). For this first note that

$$
I_{2}^{!} \circ \operatorname{Res}_{\tau} \circ \tilde{I}_{2} \cong \operatorname{Res}_{\tau_{2}} \circ \tilde{I}_{2}^{!} \circ \tilde{I}_{2} \cong \operatorname{Res}_{\tau_{2}}
$$

by part (c) and full faithfulness of $\tilde{I}_{2}$. Therefore, for any $N_{2} \in \mathbf{D}\left(\tilde{\mathscr{D}}_{2}\right)$ the component of $\operatorname{Res}_{\tau}\left(\tilde{I}_{2}\left(N_{2}\right)\right)$ in $\mathbf{D}\left(\mathscr{D}_{2}\right)$ equals $\operatorname{Res}_{\tau_{2}}\left(N_{2}\right)$. Hence its decomposition triangle looks as

$$
I_{2}\left(\operatorname{Res}_{\tau_{2}}\left(N_{2}\right)\right) \rightarrow \operatorname{Res}_{\tau}\left(\tilde{I}_{2}\left(N_{2}\right)\right) \rightarrow I_{1}\left(M_{1}\right)
$$

for some $M_{1} \in \mathbf{D}\left(\tilde{\mathscr{D}}_{1}\right)$. It remains to show that $M_{1}=0$. For this, we apply the functor $I_{1}^{!}$to the above triangle. Since $I_{1}$ is fully faithful, we obtain

$$
I_{1}^{!}\left(I_{2}\left(\operatorname{Res}_{\tau_{2}}\left(N_{2}\right)\right)\right) \rightarrow I_{1}^{!}\left(\operatorname{Res}_{\tau}\left(\tilde{I}_{2}\left(N_{2}\right)\right)\right) \rightarrow M_{1} .
$$


Note that $I_{1}^{!} \circ I_{2} \cong L \varphi[-1]$ by Proposition 4.6, hence the first term is $L \varphi\left(\operatorname{Res}_{\tau_{2}}\left(N_{2}\right)\right)[-1]$. On the other hand, $I_{1}^{!} \circ \operatorname{Res}_{\tau} \circ \tilde{I}_{2} \cong \operatorname{Res}_{\tau_{1}} \circ \tilde{I}_{1}^{!} \circ \tilde{I}_{2} \cong \operatorname{Res}_{\tau_{1}} \circ L \tilde{\varphi}[-1]$ (the first is by part (c) and the second again by Proposition 4.6). So, if the canonical morphism $L \varphi \circ \operatorname{Res}_{\tau_{2}} \rightarrow$ $\operatorname{Res}_{\tau_{1}} \circ L \tilde{\varphi}$ is an isomorphism, then $M_{1}=0$ and we are done.

Remark 4.12. Note that if $\tau_{2}=\mathrm{id}$, then the condition of part (e) follows from (a). So, in this case the claim of part (e) holds.

Now assume that $\varphi \in\left(\mathscr{D}_{2}^{\mathrm{op}} \otimes \mathscr{D}_{1}\right)$-dgm is an h-projective bimodule (recall that by Lemma 4.7 we can replace the gluing bimodule by its h-projective resolution without changing the quasiequivalence class of the resulting category) and define the bimodule $\tilde{\varphi} \in\left(\tilde{\mathscr{D}}_{2}^{\mathrm{op}} \otimes \tilde{\mathscr{D}}_{1}\right)$-dgm by

$$
\tilde{\varphi}=\tilde{\mathscr{D}}_{2 \tau_{2}} \otimes_{\mathscr{D}_{2}} \varphi \otimes_{\mathscr{D}_{1}} \tau_{1} \tilde{\mathscr{D}}_{1}
$$

Note that, by definition of a DG-functor, we have the canonical morphisms of bimodules

$$
\tau_{1}: \mathscr{D}_{1} \rightarrow{ }_{\tau_{1}} \tilde{\mathscr{D}}_{1 \tau_{1}} \quad \text { and } \quad \tau_{2}: \mathscr{D}_{2} \rightarrow{ }_{\tau_{2}} \tilde{\mathscr{D}}_{2 \tau_{2}}
$$

which are quasiisomorphisms since $\tau_{1}$ and $\tau_{2}$ are quasi-fully faithful. Tensoring with $\varphi$, we obtain a morphism

$$
C: \varphi=\mathscr{D}_{2} \otimes_{\mathscr{D}_{2}} \varphi \otimes_{\mathscr{D}_{1}} \mathscr{D}_{1} \stackrel{\tau_{2} \otimes 1 \otimes \tau_{1}}{\longrightarrow} \tau_{2} \tilde{\mathscr{D}}_{2 \tau_{2}} \otimes_{\mathscr{D}_{2}} \varphi \otimes_{\mathscr{D}_{1}} \tilde{\tau}_{1} \tilde{\mathscr{D}}_{1 \tau_{1}}={ }_{\tau_{2}} \tilde{\varphi}_{\tau_{1}}
$$

Note that since $\varphi$ is h-projective, this map is a quasiisomorphism, so $\tilde{\varphi}$ is compatible with $\varphi$. We call the gluing $\tilde{\mathscr{D}}=\tilde{\mathscr{D}}_{1} \times_{\tilde{\varphi}} \tilde{\mathscr{D}}_{2}$ with $\tilde{\varphi}$ defined by (29) the regluing of $\mathscr{D}$. By Proposition 4.11 , the regluing $\tilde{\mathscr{D}}$ is a partial categorical DG-resolution of $\mathscr{D}$. The following proposition describes its properties.

Proposition 4.13. Let $\tilde{\mathscr{D}}=\tilde{\mathscr{D}}_{1} \times_{\tilde{\varphi}} \tilde{\mathscr{D}}_{2}$ be the regluing of $\mathscr{D}=\mathscr{D}_{1} \times_{\varphi} \mathscr{D}_{2}$. Then, we have

(a) $L \tilde{\varphi} \cong \operatorname{LInd}_{\tau_{1}} \circ L \varphi \circ \operatorname{Res}_{\tau_{2}}$, an isomorphism of functors $\mathbf{D}\left(\tilde{\mathscr{D}}_{2}\right) \rightarrow \mathbf{D}\left(\tilde{\mathscr{D}}_{1}\right)$;

(b) $\operatorname{Res}_{\tau} \circ \tilde{I}_{k} \cong I_{k} \circ \operatorname{Res}_{\tau_{1}}$ for $k=1,2$, an isomorphism of functors $\mathbf{D}\left(\tilde{\mathscr{D}}_{k}\right) \rightarrow$ $\mathbf{D}(\mathscr{D})$.

Proof. The first property is straightforward

$$
\begin{aligned}
& L \tilde{\varphi}(M)=M \stackrel{\mathbb{L}}{\otimes} \tilde{\mathscr{D}}_{2} \tilde{\varphi}=M \stackrel{\mathbb{L}}{\otimes} \tilde{\mathscr{D}}_{2}\left(\tilde{\mathscr{D}}_{2 \tau_{2}} \stackrel{\mathbb{L}}{\otimes} \mathscr{D}_{2} \varphi \stackrel{\mathbb{L}}{\otimes} \mathscr{D}_{1} \tau_{1} \tilde{\mathscr{D}}_{1}\right) \\
& \cong M_{\tau_{2}} \stackrel{\mathbb{L}}{\otimes} \mathscr{D}_{2} \varphi \stackrel{\mathbb{L}}{\otimes} \mathscr{D}_{1} \tau_{1} \tilde{\mathscr{D}}_{1} \cong \operatorname{LInd}_{\tau_{1}}\left(L \varphi\left(\operatorname{Res}_{\tau_{2}}(M)\right)\right),
\end{aligned}
$$


which is precisely the required formula. For (b) note that the case $k=1$ is given by Proposition $4.11(\mathrm{~d})$, so it remains to consider the case $k=2$. For this, we note that

$$
\operatorname{Res}_{\tau_{1}} \circ L \tilde{\varphi} \cong \operatorname{Res}_{\tau_{1}} \circ \operatorname{LInd}_{\tau_{1}} \circ L \varphi \circ \operatorname{Res}_{\tau_{2}} \cong L \varphi \circ \operatorname{Res}_{\tau_{2}}
$$

so the condition of Proposition 4.11(e) is satisfied. Hence, we have the required isomorphism by Proposition 4.11(e).

The following result is not needed for the rest of the paper, but we add it for completeness.

Proposition 4.14. In the assumptions of Proposition 4.11 the functor

$$
\tau: \mathscr{D}_{1} \times_{\varphi} \mathscr{D}_{2} \rightarrow \tilde{\mathscr{D}}_{1} \times_{\tilde{\varphi}} \tilde{\mathscr{D}}_{2}
$$

is a quasiequivalence if and only if both $\tau_{1}$ and $\tau_{2}$ are quasiequivalences.

Proof. Assume that both $\tau_{1}$ and $\tau_{2}$ are quasiequivalences. Since we already know that $\tau$ is quasi-fully faithful, it only suffices to check that it is essentially surjective on the homotopy categories. So, let $\left(\tilde{M}_{1}, \tilde{M}_{2}, \tilde{\mu}\right)$ be an object of $\tilde{\mathscr{D}}_{1} \times_{\tilde{\varphi}} \tilde{\mathscr{D}}_{2}$. Since $\tau_{1}$ and $\tau_{2}$ are quasiequivalences, there are objects $M_{1} \in \mathscr{D}_{1}$ and $M_{2} \in \mathscr{D}_{2}$ such that $\tilde{M}_{1}$ is homotopic to $\tau_{1}\left(M_{1}\right)$ and $\tilde{M}_{2}$ is homotopic to $\tau_{2}\left(M_{2}\right)$. The above homotopies induce a homotopy equivalence of $\tilde{\varphi}\left(\tilde{M}_{2}, \tilde{M}_{1}\right)$ and $\tilde{\varphi}\left(\tau_{2}\left(M_{2}\right), \tau_{1}\left(M_{1}\right)\right)$. The latter by assumption is quasiisomorphic to $\varphi\left(M_{2}, M_{1}\right)$. Thus, we can find a closed element of zero degree $\mu \in \varphi\left(M_{2}, M_{1}\right)$ such that $c(\mu)$ goes to $\tilde{\mu}$ under the homotopy equivalence of the complexes mentioned above. It is quite easy to check then that $\tau\left(M_{1}, M_{2}, \mu\right)$ is homotopic to $\left(\tilde{M}_{1}, \tilde{M}_{2}, \tilde{\mu}\right)$, so $\tau$ is essentially surjective on the homotopy categories.

Conversely, assume that $\tau$ is a quasiequivalence. We have to check that both $\tau_{1}$ and $\tau_{2}$ are essentially surjective on homotopy categories. Take any $\tilde{M}_{1} \in \tilde{\mathscr{D}}_{1}$. Then, $\left(\tilde{M}_{1}, 0,0\right)$ is an object of $\tilde{\mathscr{D}}_{1} \times_{\tilde{\varphi}} \tilde{\mathscr{D}}_{2}$. Hence, there is an object $\left(M_{1}, M_{2}, \mu\right)$ of $\mathscr{D}_{1} \times_{\varphi} \mathscr{D}_{2}$ such that $\tau\left(M_{1}, M_{2}, \mu\right)$ is homotopic to $\left(\tilde{M}_{1}, 0,0\right)$. By definition of the gluing it follows that $\tilde{M}_{1}$ is homotopic to $\tau_{1}\left(M_{1}\right)$. Thus $\tau_{1}$ is essentially surjective on homotopy categories. Analogously, one considers $\tau_{2}$. 


\section{Partial Categorical Resolution of a Nonreduced Scheme}

The goal of this section is to construct a categorical resolution for a nonreduced scheme for which the corresponding reduced scheme is smooth. Our construction is inspired by the result of Auslander [2].

\section{$5.1 \mathscr{A}$-modules}

Let $S$ be a separated nonreduced scheme of finite type over $\mathbb{k}$. Let $\mathfrak{r} \subset \mathcal{O}_{S}$ be a nilpotent sheaf of ideals. Choose an integer $n$ such that

$$
\mathfrak{r}^{n}=0 .
$$

Note that we do not require $n$ to be the minimal integer with this property. In particular, we can take $\mathfrak{r}=0$ and arbitrary $n \geq 1$.

Consider a sheaf of noncommutative algebras

$$
\mathscr{A}=\mathscr{A}_{S, \mathfrak{r}, n}:=\left(\begin{array}{ccccc}
\mathcal{O}_{S} & \mathfrak{r} & \mathfrak{r}^{2} & \ldots & \mathfrak{r}^{n-1} \\
\mathcal{O}_{S} / \mathfrak{r}^{n-1} & \mathcal{O}_{S} / \mathfrak{r}^{n-1} & \mathfrak{r} / \mathfrak{r}^{n-1} & \ldots & \mathfrak{r}^{n-2} / \mathfrak{r}^{n-1} \\
\mathcal{O}_{S} / \mathfrak{r}^{n-2} & \mathcal{O}_{S} / \mathfrak{r}^{n-2} & \mathcal{O}_{S} / \mathfrak{r}^{n-2} & \ldots & \mathfrak{r}^{n-3} / \mathfrak{r}^{n-2} \\
\vdots & \vdots & \vdots & \ddots & \vdots \\
\mathcal{O}_{S} / \mathfrak{r} & \mathcal{O}_{S} / \mathfrak{r} & \mathcal{O}_{S} / \mathfrak{r} & \ldots & \mathcal{O}_{S} / \mathfrak{r}
\end{array}\right)
$$

with the algebra structure induced by the embedding

$$
\mathscr{A}_{S, \mathfrak{r}, n} \subset \operatorname{End}_{\mathcal{O}_{S}}\left(\mathcal{O}_{S} \oplus \mathcal{O}_{S} / \mathfrak{r}^{n-1} \oplus \mathcal{O}_{S} / \mathfrak{r}^{n-2} \oplus \cdots \oplus \mathcal{O}_{S} / \mathfrak{r}\right)
$$

In other words, $\mathscr{A}_{i j}=\mathfrak{r}^{\max (j-i, 0)} / \mathfrak{r}^{n+1-i}$ and the multiplication is the map

$$
\mathscr{A}_{i j} \otimes \mathscr{A}_{j k}=\left(\mathfrak{r}^{\max (j-i, 0)} / \mathfrak{r}^{n+1-i}\right) \otimes\left(\mathfrak{r}^{\max (k-j, 0)} / \mathfrak{r}^{n+1-j}\right) \rightarrow \mathfrak{r}^{\max (k-i, 0)} / \mathfrak{r}^{n+1-i}=\mathscr{A}_{i k}
$$

induced by the multiplication map $\mathfrak{r}^{a} \otimes \mathfrak{r}^{b} \rightarrow \mathfrak{r}^{c}$, which is defined for all $c \leq a+b$-we note that, in our case, this condition is satisfied since

$$
\max (k-i, 0) \leq \max (j-i, 0)+\max (k-j, 0) .
$$

Also note that

$$
\max (j-i, 0)+(n+1-j) \geq n+1-i \text { and } \max (k-j, 0)+(n+1-i) \geq n+1-i,
$$

so the above map descends to a map of the quotients. 
We define

$$
\epsilon_{i}=1 \in \mathscr{A}_{i i}=\mathcal{O}_{S} / \mathfrak{r}^{n+1-i}
$$

Note that $\epsilon_{1}, \ldots, \epsilon_{n}$ is a system of orthogonal idempotents with

$$
\epsilon_{1}+\cdots+\epsilon_{n}=1
$$

The algebra $\mathscr{A}=\mathscr{A}_{S, \mathfrak{r}, n}$ will be called the Auslander algebra ( $\mathscr{A}$ is for Auslander!). The scheme $S$ will be referred to as the underlying scheme, the ideal $\mathfrak{r}$ as the defining ideal, and the integer $n$ as the width of the Auslander algebra.

Remark 5.1. Auslander [2], in the case of a zero-dimensional scheme $S$, considered the algebra $\operatorname{End}_{\mathcal{O}_{S}}\left(\mathcal{O}_{S} \oplus \mathcal{O}_{S} / \mathfrak{r}^{n-1} \oplus \mathcal{O}_{S} / \mathfrak{r}^{n-2} \oplus \cdots \oplus \mathcal{O}_{S} / \mathfrak{r}\right)$ and showed that it has finite global dimension. One can show (see Section A.6) that a similar statement is true for the algebra $\mathscr{A}$ for the scheme $S$ of arbitrary dimension as soon as the reduced scheme $S_{\text {red }}$ is smooth (note that this holds automatically if $S$ is zero-dimensional). Moreover, we show in Section A.7 that there is a more general class of generalized Auslander algebras that have similar properties. Now, we just note that the algebra we consider coincides with the original Auslander algebra if and only if $\mathfrak{r}^{a}: \mathfrak{r}^{b}=\mathfrak{r}^{a-b}$ for all $a>b$ (here $\mathfrak{r}^{a}: \mathfrak{r}^{b}$ is the ideal consisting of all $x \in \mathcal{O}_{S}$ such that $x \mathfrak{r}^{b} \subset \mathfrak{r}^{a}$ ).

In what follows, we always consider right $\mathscr{A}$-modules, unless the opposite is specified.

An $\mathscr{A}$-module is called quasicoherent (coherent) if it is such as an $\mathcal{O}$-module. We denote by $\operatorname{Ccoh}(\mathscr{A})$ the category of quasicoherent $\mathscr{A}$-modules on $S$ and by $\operatorname{coh}(\mathscr{A})$ its subcategory of coherent $\mathscr{A}$-modules.

Remark 5.2. Note that, taking $\mathfrak{r}=0$ and $n=1$, we obtain

$$
\mathscr{A}_{S, 0,1} \cong \mathcal{O}_{S} .
$$

Thus, the category of $\mathcal{O}_{S}$-modules is just a very special case of the category of $\mathscr{A}$-modules.

Example 5.3. A simple but rather instructive example is the case of $S=\operatorname{Spec} \mathbb{k}[t] / t^{2}$, $\mathfrak{r}=t \mathbb{k}[t] / t^{2}, n=2$. In this case, the category $\operatorname{Qcoh}\left(\mathscr{A}_{S}\right)$ is the category of representations 
of the quiver with relation

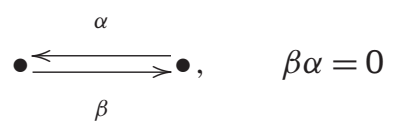

and $\mathscr{A}_{S, \mathfrak{r}, 2}$ is just the path algebra of this quiver. On the other hand, if we take the same $S, \mathfrak{r}$, and choose $n=3$, we will get a quiver

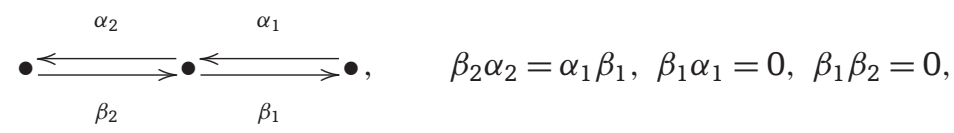

and $\mathscr{A}_{S, \mathfrak{r}, 3}$ is the path algebra of this quiver.

\subsection{The category of $\mathscr{A}$-spaces}

An $\mathscr{A}$-space is a triple $\left(S, \mathfrak{r}_{S}, n_{S}\right)$, where $S$ is a scheme, $\mathfrak{r}$ is a nilpotent ideal in $\mathcal{O}_{S}$, and $n_{S}$ is an integer such that $\mathfrak{r}^{n}=0$. An $\mathscr{A}$-space $\left(S, \mathfrak{r}_{S}, n_{S}\right)$ can be thought of as a ringed space with the underlying topological space of the scheme $S$ and the sheaf of (noncommutative) rings $\mathscr{A}_{S}=\mathscr{A}_{S, \mathfrak{r}, n_{S}}$. Sometimes we will denote an $\mathscr{A}$-space $\left(S, \mathfrak{r}_{S}, n_{S}\right)$ simply by $\left(S, \mathcal{A}_{S}\right)$. With any $\mathscr{A}$-space we associate the category Qcoh( $\mathscr{A})$ of quasicoherent sheaves of right $\mathscr{A}$-modules.

There are several ways how one can define morphisms of $\mathscr{A}$-spaces. Here, we will use the simplest possible definition. A more general definition will be discussed in Section A.8.

Assume that $\left(S, \mathfrak{r}_{S}, n_{S}\right)$ and $\left(T, \mathfrak{r}_{T}, n_{T}\right)$ is a pair of $\mathscr{A}$-spaces and let $f: T \rightarrow S$ be a morphism of underlying schemes. Assume that the defining ideals $\mathfrak{r}_{T}$ and $\mathfrak{r}_{S}$ and the widths $n_{T}$ and $n_{S}$ are compatible in the following sense:

$$
f^{-1}\left(\mathfrak{r}_{S}\right) \subset \mathfrak{r}_{T} \text { and } n_{S} \leq n_{T}
$$

It follows from (33) that $f^{-1}\left(\mathfrak{r}_{S}^{i}\right) \subset \mathfrak{r}_{T}^{i}$ for all $i$. Therefore, the morphism of schemes induces maps

$$
f^{-1}\left(\mathfrak{r}_{S}^{i} / \mathfrak{r}_{S}^{j}\right)=f^{-1}\left(\mathfrak{r}_{S}^{i}\right) / f^{-1}\left(\mathfrak{r}_{S}^{j}\right) \rightarrow \mathfrak{r}_{T}^{i} / \mathfrak{r}_{T}^{j}
$$

for all $i$ and $j$ that are compatible with the multiplication maps. Combining all these together, we obtain a (nonunital) morphism of sheaves of algebras $f^{-1}\left(\mathscr{A}_{S}\right) \rightarrow \mathscr{A}_{T}$ which takes the unit of the first to the idempotent $\epsilon_{1}+\cdots+\epsilon_{n_{S}}$ of the second. In particular, $\left(\epsilon_{1}+\cdots+\epsilon_{n_{S}}\right) \mathscr{A}_{T}$ has a natural structure of an $f^{-1} \mathscr{A}_{S^{-}} \mathscr{A}_{T}$-bimodule which is projective over $\mathscr{A}_{T}$. 
We define the pullback and the pushforward functors with respect to a morphism $f$ by

$$
\begin{aligned}
& f^{*}: \operatorname{Ocoh}\left(\mathscr{A}_{S}\right) \rightarrow \operatorname{Qcoh}\left(\mathscr{A}_{T}\right), \quad M \mapsto f^{-1} M \otimes_{f^{-1} \mathscr{A}_{S}}\left(\epsilon_{1}+\cdots+\epsilon_{n_{S}}\right) \mathscr{A}_{T}, \\
& f_{*}: \operatorname{Ocoh}\left(\mathscr{A}_{T}\right) \rightarrow \operatorname{Qcoh}\left(\mathscr{A}_{S}\right), \quad N \mapsto\left(f_{*} N\right)\left(\epsilon_{1}+\cdots+\epsilon_{n_{S}}\right) .
\end{aligned}
$$

Lemma 5.4. The functor $f^{*}$ is right exact and the functor $f_{*}$ is left exact. Moreover, the functor $f^{*}$ is the left adjoint of the functor $f_{*}$.

Proof. The functor $f^{*}$ is the composition of the exact functor $f^{-1}$ and the right exact functor of tensor product, so it is right exact. Analogously, the functor $f_{*}$ is the composition of the left exact functor $f_{*}$ and of the exact functor $N \mapsto N\left(\epsilon_{1}+\cdots+\epsilon_{n_{S}}\right)$. Finally, the adjunction is classical

$$
\begin{aligned}
\operatorname{Hom}_{\mathscr{A}_{T}}\left(f^{*} M, N\right) & =\operatorname{Hom}_{\mathscr{A}_{T}}\left(f^{-1} M \otimes_{f^{-1} \mathscr{A}_{S}}\left(\epsilon_{1}+\cdots+\epsilon_{n_{S}}\right) \mathscr{A}_{T}, N\right) \\
& \cong \operatorname{Hom}_{f^{-1} \mathscr{A}_{S}}\left(f^{-1} M, \operatorname{Hom}_{\mathscr{A}_{T}}\left(\left(\epsilon_{1}+\cdots+\epsilon_{n_{S}}\right) \mathscr{A}_{T}, N\right)\right) \\
& \cong \operatorname{Hom}_{f^{-1} \mathscr{A}_{S}}\left(f^{-1} M, N\left(\epsilon_{1}+\cdots+\epsilon_{n_{S}}\right) \cong \operatorname{Hom}_{\mathscr{A}_{S}}\left(M, f_{*} N\left(\epsilon_{1}+\cdots+\epsilon_{n_{S}}\right)\right) .\right.
\end{aligned}
$$

Here, the first isomorphism is the classical adjunction of Hom and $\otimes$, the second is evident, and the third is the classical adjunction of $f^{-1}$ and $f_{*}$.

Lemma 5.5. Assume that

$$
\left(U, \mathfrak{r}_{U}, n_{U}\right) \stackrel{g}{\longrightarrow}\left(T, \mathfrak{r}_{T}, n_{T}\right) \stackrel{f}{\longrightarrow}\left(S, \mathfrak{r}_{S}, n_{S}\right)
$$

are morphisms of $\mathscr{A}$-spaces. Then, there is an isomorphism of functors $g^{*} \circ f^{*} \cong(f \circ g)^{*}$ from $\operatorname{Qcoh}\left(\mathscr{A}_{S}\right)$ to $\operatorname{Qcoh}\left(\mathscr{A}_{U}\right)$.

Proof. Indeed,

$$
\begin{aligned}
g^{*}\left(f^{*}(F)\right) & =g^{-1}\left(f^{-1} F \otimes_{f^{-1} \mathscr{A}_{S}}\left(\epsilon_{1}+\cdots+\epsilon_{n_{S}}\right) \mathscr{A}_{T}\right) \otimes_{g^{-1} \mathscr{A}_{T}}\left(\epsilon_{1}+\cdots+\epsilon_{n_{T}}\right) \mathscr{A}_{U} \\
& \cong g^{-1} f^{-1} F \otimes_{g^{-1} f^{-1} \mathscr{A}_{S}}\left(\left(\epsilon_{1}+\cdots+\epsilon_{n_{S}}\right) g^{-1} \mathscr{A}_{T} \otimes_{g^{-1} \mathscr{A}_{T}}\left(\epsilon_{1}+\cdots+\epsilon_{n_{T}}\right) \mathscr{A}_{U}\right) \\
& \cong g^{-1} f^{-1} F \otimes_{g^{-1} f^{-1} \mathscr{A}_{S}}\left(\epsilon_{1}+\cdots+\epsilon_{n_{S}}\right) \mathscr{A}_{U}=(f \circ g)^{*}(F)
\end{aligned}
$$

since $\left(\epsilon_{1}+\cdots+\epsilon_{n_{S}}\right)\left(\epsilon_{1}+\cdots+\epsilon_{n_{T}}\right)=\epsilon_{1}+\cdots+\epsilon_{n_{S}}$ by (33). 


\subsection{Derived category}

Let $\operatorname{com}(\mathscr{A})$ denote the category of (unbounded) complexes of quasicoherent $\mathscr{A}$-modules.

\section{Lemma 5.6.}

(1) The category $\mathrm{Q} \operatorname{coh}(\mathscr{A})$ is a Grothendieck category.

(2) The category $\operatorname{com}(\mathscr{A})$ has enough h-injective complexes.

(3) The category $\operatorname{com}(\mathscr{A})$ has enough h-flat complexes.

Proof. Note that all these assertions hold for the category $\operatorname{Qcoh}(S)$ instead of $\operatorname{Qcoh}(\mathscr{A})$.

(1) The category $\operatorname{Qcoh}(\mathscr{A})$ has arbitrary direct sums and filtered direct limits in $\operatorname{Qcoh}(\mathscr{A})$ are exact. It remains to show that it has a generator. Recall that $\mathscr{A}$ is an $\mathcal{O}_{S}$-algebra. Consider the corresponding extension and restriction of scalars functors

$$
\begin{aligned}
& \operatorname{Ind}_{S}: \operatorname{Qcoh}(S) \rightarrow \operatorname{Qcoh}(\mathscr{A}), \quad \mathcal{M} \mapsto \mathcal{M} \otimes_{\mathcal{O}_{S}} \mathscr{A}, \\
& \operatorname{Res}_{S}: \operatorname{Ocoh}(\mathscr{A}) \rightarrow \operatorname{Qcoh}(S), \quad \mathcal{N} \mapsto \mathcal{N}_{\mathcal{O}_{S}} .
\end{aligned}
$$

It is a classical fact that $\operatorname{Ind}_{S}$ is the left adjoint of $\operatorname{Res}_{S}$.

Let $U \in \operatorname{Ocoh}(S)$ be a generator. We claim that $\operatorname{Ind}_{S}(U)$ is a generator of $\operatorname{Qcoh}(\mathscr{A})$. Indeed, let $\mathcal{M}_{0} \varsubsetneqq \mathcal{M}$ be objects in $\operatorname{Qcoh}(\mathscr{A})$. Then $\operatorname{Res}_{S}\left(\mathcal{M}_{0}\right) \varsubsetneqq \operatorname{Res}_{S}(\mathcal{M})$. Hence the map $\operatorname{Hom}\left(U, \operatorname{Res}_{S}\left(\mathcal{M}_{0}\right)\right) \rightarrow \operatorname{Hom}\left(U, \operatorname{Res}_{S}(\mathcal{M})\right)$ is not surjective. But then the map $\operatorname{Hom}\left(\operatorname{Ind}_{S}(U), \mathcal{M}_{0}\right) \rightarrow \operatorname{Hom}\left(\operatorname{Ind}_{S}(U), \mathcal{M}\right)$ is also not surjective. So $\operatorname{Ind}_{S}(U)$ is a generator and hence $\operatorname{Qcoh}(\mathscr{A})$ is a Grothendieck category.

(2) This follows from (1) and [13].

(3) The proof given in [1] for the category $\operatorname{Qcoh}(S)$ works also for $\operatorname{Qcoh}(\mathcal{B})$ for any sheaf of $\mathcal{O}_{S}$-algebras $\mathcal{B}$ which is quasicoherent as an $\mathcal{O}_{S}$-module.

We denote by ASch the category of $\mathscr{A}$-spaces with the underlying scheme being separated of finite type and with morphisms defined in Section 5.2. As we already observed, each scheme can be thought of as an $\mathscr{A}$-space of width 1 and each morphism of schemes gives a morphism of the corresponding $\mathscr{A}$-spaces. Thus, the category Sch is a subcategory of ASch. Our goal is to extend the derived category pseudofunctor D : Sch ${ }^{\text {op }} \rightarrow$ Tria to the category ASch.

As we know by Lemma 5.6, the category $\operatorname{com}\left(\mathrm{O} \operatorname{coh}\left(\mathscr{A}_{S}\right)\right)$ has enough h-flat objects. Hence $\left[\operatorname{com}\left(\mathscr{A}_{S}\right)\right] /\left[\operatorname{com}^{\circ}\left(\mathscr{A}_{S}\right)\right] \cong\left[\mathrm{h}-\mathrm{flat}\left(\mathscr{A}_{S}\right)\right] /\left[\mathrm{h}-\right.$ flat $\left.^{\circ}\left(\mathscr{A}_{S}\right)\right]$, where $\operatorname{com}^{\circ}\left(\mathscr{A}_{S}\right) \subset \operatorname{com}\left(\mathscr{A}_{S}\right)$ is the DG-subcategory of acyclic complexes, h-flat $\left(\mathscr{A}_{S}\right)$ is the DG-category of h-flat complexes 
of $\mathscr{A}_{S}$-modules, and $\mathrm{h}$-flat ${ }^{\circ}\left(\mathscr{A}_{S}\right)$ is its DG-subcategory of h-flat acyclic complexes. So, analogously to the case of schemes we can identify the derived category as

$$
\mathbf{D}\left(\mathscr{A}_{S}\right) \cong\left[\mathrm{h}-\text { flat }\left(\mathscr{A}_{S}\right) / \mathrm{h}^{-f l a t}{ }^{\circ}\left(\mathscr{A}_{S}\right)\right],
$$

the homotopy category of the Drinfeld quotient. It is clear that $\mathbf{D}^{b}\left(\operatorname{coh}\left(\mathscr{A}_{S}\right)\right)$, the bounded derived category of coherent $\mathscr{A}_{S}$-modules, is equivalent to the subcategory of $\mathbf{D}\left(\mathscr{A}_{S}\right)$ consisting of complexes with finite number of cohomology sheaves, all of which are coherent. Indeed, by a standard argument (see [3, Expose II, Proposition 2.2.2]) it is enough to check that a quotient of a coherent $\mathscr{A}_{S}$-module is coherent and that any quasicoherent $\mathscr{A}_{S}$-module is a filtered direct limit of coherent $\mathscr{A}_{S}$-modules. The first is evident and, for the second, note that if $\mathcal{N}$ is an $\mathscr{A}_{S}$-module and $\operatorname{Res} \mathcal{N}=\lim \mathcal{M}_{\lambda}$ with $\mathcal{M}_{\lambda}$ coherent sheaves on $S$, then $\mathcal{N}$ is the limit of images of $\operatorname{Ind}\left(\mathcal{M}_{\lambda}\right)$ and each of those is a coherent $\mathscr{A}_{S}$-module.

Proposition 5.7. Associating with an $\mathscr{A}$-space $\left(S, \mathscr{A}_{S}\right)$ its derived category $\mathbf{D}\left(\mathscr{A}_{S}\right)$ is a pseudofunctor $\mathrm{ASch}^{\mathrm{op}} \rightarrow$ Tria from the category opposite to $\mathscr{A}$-spaces to the 2-category of triangulated categories, extending the same-named pseudofunctor Sch $^{\text {op }} \rightarrow$ Tria.

Proof. To define a pseudofunctor ASch ${ }^{\mathrm{op}} \rightarrow$ Tria, we have to associate with a morphism $f:\left(T, \mathscr{A}_{T}\right) \rightarrow\left(S, \mathscr{A}_{S}\right)$ of $\mathscr{A}$-spaces a functor $\mathbf{D}\left(\mathscr{A}_{S}\right) \rightarrow \mathbf{D}\left(\mathscr{A}_{T}\right)$. This role will be played by the derived pullback functor $L f^{*}$; which we will define along the same lines as the definition of the usual derived pullback functor in Section 3.10.

Using an analog of Lemma 3.10, we deduce that the functor $f^{*}$ takes h-flat $\left(\mathscr{A}_{S}\right)$ to h-flat $\left(\mathscr{A}_{T}\right)$ and h-flat ${ }^{\circ}\left(\mathscr{A}_{S}\right)$ to h-flat ${ }^{\circ}\left(\mathscr{A}_{T}\right)$, and hence, by Proposition 3.7, induces a DGfunctor h-flat $\left(\mathscr{A}_{S}\right) /$ h-flat $^{\circ}\left(\mathscr{A}_{S}\right) \rightarrow$ h-flat $\left(\mathscr{A}_{T}\right) /$ h-flat ${ }^{\circ}\left(\mathscr{A}_{T}\right)$ of Drinfeld quotients. Passing to homotopy categories, we define the derived functor

$$
L f^{*}: \mathbf{D}\left(\mathscr{A}_{S}\right)=\left[\mathrm{h}-f \text { lat }\left(\mathscr{A}_{S}\right) / \mathrm{h}-\operatorname{flat}^{\circ}\left(\mathscr{A}_{S}\right)\right] \stackrel{f^{*}}{\longrightarrow}\left[\mathrm{h}-f \text { lat }\left(\mathscr{A}_{T}\right) / \mathrm{h}-\text { flat }^{\circ}\left(\mathscr{A}_{T}\right)\right]=\mathbf{D}\left(\mathscr{A}_{T}\right) .
$$

The pseudofunctoriality and compatibility with the derived category pseudofunctor on schemes are evident.

Let $f:\left(T, \mathscr{A}_{T}\right) \rightarrow\left(S, \mathscr{A}_{S}\right)$ be a morphism of $\mathscr{A}$-spaces. By construction the derived pullback functor $L f^{*}$ commutes with arbitrary direct sums. Therefore, by Brown Representability it has a right adjoint functor $R f_{*}: \mathbf{D}\left(\mathscr{A}_{T}\right) \rightarrow \mathbf{D}\left(\mathscr{A}_{S}\right)$.

For each $1 \leq k \leq n_{S}$ consider a slight generalization of the induction functor $\operatorname{Ind}_{S}^{k}: \operatorname{Qcoh}(S) \rightarrow \operatorname{Qcoh}\left(\mathscr{A}_{S}\right), M \mapsto M \otimes_{\mathcal{O}_{S}}\left(\epsilon_{1}+\cdots+\epsilon_{k}\right) \mathscr{A}_{S}$. Its right adjoint functor $\operatorname{Res}_{S}^{k}:$ 
$\operatorname{Qcoh}\left(\mathscr{A}_{S}\right) \rightarrow \operatorname{Qcoh}(S)$ is given by $N \mapsto \operatorname{Res}_{S}\left(N\left(\epsilon_{1}+\cdots+\epsilon_{k}\right)\right)$. Note that it is exact. The above argument allows one to define the derived induction functor $\operatorname{LInd}_{S}^{k}: \mathbf{D}(S) \rightarrow \mathbf{D}\left(\mathscr{A}_{S}\right)$ by applying $\operatorname{Ind}_{S}^{k}$ to h-flat complexes. On the other hand, the exactness of the restriction functor shows that it descends to the derived category $\operatorname{Res}_{S}^{k}: \mathbf{D}\left(\mathscr{A}_{S}\right) \rightarrow \mathbf{D}(S)$ and the adjunction is preserved.

Lemma 5.8. If $f:\left(T, \mathfrak{r}_{T}, n_{T}\right) \rightarrow\left(S, \mathfrak{r}_{S}, n_{S}\right)$ is a morphism of $\mathscr{A}$-spaces, then

$$
\operatorname{Res}_{S}\left(R f_{*}(F)\right) \cong R f_{*}\left(\operatorname{Res}_{T}^{n_{S}}(F)\right)
$$

In particular, if $f$ is proper and of finite type, then $R f_{*}\left(\mathbf{D}^{b}\left(\operatorname{coh}\left(\mathscr{A}_{T}\right)\right) \subset \mathbf{D}^{b}\left(\operatorname{coh}\left(\mathscr{A}_{S}\right)\right)\right.$.

Proof. Both sides are compositions of right adjoint functors, so it is enough to check an isomorphism of their left adjoint functors $L f^{*} \circ \operatorname{LInd}_{S} \cong \operatorname{LInd}_{T}^{n_{S}} \circ L f^{*}$. Since the induction of an h-flat complex of $\mathcal{O}_{S}$-modules is evidently h-flat over $\mathscr{A}_{S}$, it is enough to note that we have an isomorphism for underived functors. The latter is clear as

$$
\begin{aligned}
f^{*}\left(\operatorname{Ind}_{S}(M)\right)=f^{*}\left(M \otimes_{\mathcal{O}_{S}} \mathscr{A}_{S}\right) & =f^{-1}\left(M \otimes_{\mathcal{O}_{S}} \mathscr{A}_{S}\right) \otimes_{f^{-1} \mathscr{A}_{S}}\left(\epsilon_{1}+\cdots+\epsilon_{n_{S}}\right) \mathscr{A}_{T} \\
& \cong f^{-1} M \otimes_{f^{-1} \mathcal{O}_{S}} f^{-1} \mathscr{A}_{S} \otimes_{f^{-1} \mathscr{A}_{S}}\left(\epsilon_{1}+\cdots+\epsilon_{n_{S}}\right) \mathscr{A}_{T} \\
& \cong f^{-1} M \otimes_{f^{-1} \mathcal{O}_{S}}\left(\epsilon_{1}+\cdots+\epsilon_{n_{S}}\right) \mathscr{A}_{T} \\
& \cong f^{-1} M \otimes_{f^{-1} \mathcal{O}_{S}} \mathcal{O}_{T} \otimes_{\mathcal{O}_{T}}\left(\epsilon_{1}+\cdots+\epsilon_{n_{S}}\right) \mathscr{A}_{T}=\operatorname{Ind}_{T}^{n_{S}}\left(f^{*} M\right) .
\end{aligned}
$$

The second statement is clear since $M \in \mathbf{D}\left(\mathscr{A}_{T}\right)$ being bounded and coherent implies $\operatorname{Res}_{T}^{k}(M)$ is such for any $k$, and vice versa, if $\operatorname{Res}_{S}(M) \in \mathbf{D}(S)$ is bounded and coherent, then so is $M$.

\subsection{Semiorthogonal decomposition}

Let $S_{0}$ and $S^{\prime}$ be the subschemes of $S$ corresponding to the ideals $\mathfrak{r}$ and $\mathfrak{r}^{n-1}$, respectively. For both the underlying topological space is $S$ but the sheaves of rings are

$$
\mathcal{O}_{S_{0}}:=\mathcal{O}_{S} / \mathfrak{r}, \quad \text { and } \quad \mathcal{O}_{S^{\prime}}=\mathcal{O}_{S} / \mathfrak{r}^{n-1}
$$

Consider the two-sided sheaf of ideals $\mathscr{I}$ in $\mathscr{A}$ generated by the idempotent $1-\epsilon_{1}=\epsilon_{2}+\cdots+\epsilon_{n}$. 
Lemma 5.9. We have

$$
\mathscr{I}:=\mathscr{A}\left(1-\epsilon_{1}\right) \mathscr{A}=\left(\begin{array}{ccccc}
\mathfrak{r} & \mathfrak{r} & \mathfrak{r}^{2} & \ldots & \mathfrak{r}^{n-1} \\
\mathcal{O}_{S} / \mathfrak{r}^{n-1} & \mathcal{O}_{S} / \mathfrak{r}^{n-1} & \mathfrak{r} / \mathfrak{r}^{n-1} & \ldots & \mathfrak{r}^{n-2} / \mathfrak{r}^{n-1} \\
\mathcal{O}_{S} / \mathfrak{r}^{n-2} & \mathcal{O}_{S} / \mathfrak{r}^{n-2} & \mathcal{O}_{S} / \mathfrak{r}^{n-2} & \ldots & \mathfrak{r}^{n-3} / \mathfrak{r}^{n-2} \\
\vdots & \vdots & \vdots & \ddots & \vdots \\
\mathcal{O}_{S} / \mathfrak{r} & \mathcal{O}_{S} / \mathfrak{r} & \mathcal{O}_{S} / \mathfrak{r} & \ldots & \mathcal{O}_{S} / \mathfrak{r}
\end{array}\right)
$$

In particular, $\mathscr{A} / \mathscr{I} \cong \mathcal{O}_{S} / \mathfrak{r} \cong \mathcal{O}_{S_{0}}$.

Proof. The proof is straightforward.

By Lemma 5.9, the sheaf $\mathcal{O}_{S_{0}}$ has a natural structure of an algebra over $\mathscr{A}_{S}$. Thus, we have the restriction of scalars functor

$$
\text { i }: \operatorname{Ocoh}\left(S_{0}\right) \rightarrow \operatorname{Qcoh}(\mathscr{A}), \quad M \mapsto M
$$

On the other hand, consider the algebra

$$
\mathscr{A}^{\prime}=\mathscr{A}_{S^{\prime}, \mathfrak{r}, n-1}=\oplus_{i, j \geq 2} \mathscr{A}_{i j}=\left(1-\epsilon_{1}\right) \mathscr{A}\left(1-\epsilon_{1}\right)
$$

(which is just the same type algebra constructed from the scheme $S^{\prime}$ ), and note that the following $(n-1) \times n$ matrix:

$$
\left(1-\epsilon_{1}\right) \mathscr{A}=\left(\begin{array}{ccccc}
\mathcal{O}_{S} / \mathfrak{r}^{n-1} & \mathcal{O}_{S} / \mathfrak{r}^{n-1} & \mathfrak{r} / \mathfrak{r}^{n-1} & \ldots & \mathfrak{r}^{n-2} / \mathfrak{r}^{n-1} \\
\mathcal{O}_{S} / \mathfrak{r}^{n-2} & \mathcal{O}_{S} / \mathfrak{r}^{n-2} & \mathcal{O}_{S} / \mathfrak{r}^{n-2} & \ldots & \mathfrak{r}^{n-3} / \mathfrak{r}^{n-2} \\
\vdots & \vdots & \vdots & \ddots & \vdots \\
\mathcal{O}_{S} / \mathfrak{r} & \mathcal{O}_{S} / \mathfrak{r} & \mathcal{O}_{S} / \mathfrak{r} & \ldots & \mathcal{O}_{S} / \mathfrak{r}
\end{array}\right)
$$

is an $\mathscr{A}^{\prime}-\mathscr{A}$-bimodule, so we can also define a functor

$$
\mathrm{e}: \operatorname{Ocoh}\left(\mathscr{A}^{\prime}\right) \rightarrow \operatorname{Ocoh}(\mathscr{A}), \quad M \mapsto M \otimes \mathscr{A}^{\prime}\left(1-\epsilon_{1}\right) \mathscr{A}
$$

Remark 5.10. It is useful to note that the functor e takes projective $\mathscr{A}^{\prime}$-modules to projective $\mathscr{A}$-modules. This follows immediately from the fact that $\left(1-\epsilon_{1}\right) \mathscr{A}$ is projective as an $\mathscr{A}$-module. 
Lemma 5.11. The functor $\mathrm{i}$ has a left adjoint functor $\mathrm{i}^{*}: \operatorname{Qcoh}(\mathscr{A}) \rightarrow \operatorname{Qcoh}\left(S_{0}\right)$

$$
\mathrm{i}^{*}(M)=M \otimes_{\mathscr{A}} \mathcal{O}_{S_{0}},
$$

and the functor e has a right adjoint functor $\mathrm{e}^{!}: \operatorname{Qcoh}(\mathscr{A}) \rightarrow \mathrm{Qcoh}\left(\mathscr{A}^{\prime}\right)$ :

$$
\mathrm{e}^{!}(N)=N\left(1-\epsilon_{1}\right) .
$$

Proof. The left adjoint to the restriction of scalars is the extension of scalars functor, which is precisely the functor $i^{*}$. On the other hand, it is clear that

$$
\operatorname{Hom}_{\mathscr{A}}(\mathrm{e}(M), N)=\operatorname{Hom}_{\mathscr{A}}\left(M \otimes_{\mathscr{A}}\left(1-\epsilon_{1}\right) \mathscr{A}, N\right) \cong \operatorname{Hom}_{\mathscr{A}}\left(M, \operatorname{Hom}_{\mathscr{A}}\left(\left(1-\epsilon_{1}\right) \mathscr{A}, N\right)\right),
$$

hence the right adjoint of e is given by $N \mapsto \operatorname{Hom}_{\mathscr{A}}\left(\left(1-\epsilon_{1}\right) \mathscr{A}, N\right)=N\left(1-\epsilon_{1}\right)$, which gives (41).

Remark 5.12. In fact, all these functors are just pullbacks and pushforward for generalized morphisms of $\mathscr{A}$-spaces; see Example A.25.

It is clear from (36), (39), and (41) that the functors $i, e$, and e! are exact. We extend them termwise to the categories of complexes, and to the derived categories. On the other hand, the functor $i^{*}$ is only right exact. We extend it termwise to the category of h-flat complexes of quasicoherent $\mathscr{A}$-modules and thus obtain its left derived functor $L i^{*}: \mathbf{D}(\mathscr{A}) \rightarrow \mathbf{D}\left(S_{0}\right)$,

$$
L i^{*}(M)=M \stackrel{\mathbb{L}}{\otimes} \mathcal{O}_{S_{0}}
$$

Proposition 5.13. The functor $L i^{*}: \mathbf{D}(\mathscr{A}) \rightarrow \mathbf{D}\left(S_{0}\right)$ defined by (42) is left adjoint to the functor i : $\mathbf{D}\left(S_{0}\right) \rightarrow \mathbf{D}(\mathscr{A})$, and the functor $\mathrm{e}^{!}: \mathbf{D}(\mathscr{A}) \rightarrow \mathbf{D}\left(\mathscr{A}^{\prime}\right)$ is right adjoint to e : $\mathbf{D}\left(\mathscr{A}^{\prime}\right) \rightarrow$ $\mathbf{D}(\mathscr{A})$. Moreover, we have

$$
L \mathbf{i}^{*} \circ \mathrm{i} \cong \mathrm{id}_{\mathbf{D}\left(S_{0}\right)}, \quad \mathrm{e}^{!} \circ \mathrm{i}=0, \quad L \mathbf{i}^{*} \circ \mathrm{e}=0, \quad \mathrm{e}^{!} \circ \mathrm{e} \cong \mathrm{id}_{\mathbf{D}\left(\mathscr{A}^{\prime}\right)} .
$$

In particular, the functors i and e are fully faithful.

Proof. The adjunctions of the functors on the derived level follow from those on the abelian level. So, let us verify equations (43). The first composition is the functor

$$
M \mapsto M \stackrel{\mathbb{L}}{\otimes} \mathcal{O}_{S_{0}}\left(\mathcal{O}_{S_{0}} \stackrel{\mathbb{L}}{\otimes} \mathcal{O}_{S_{0}}\right),
$$


so we have to compute the derived tensor product $\mathcal{O}_{S_{0}} \stackrel{\mathbb{L}}{\otimes} \mathcal{O}_{S_{0}}$. For this, we use the defining exact sequence

$$
0 \rightarrow \mathscr{I} \rightarrow \mathscr{A} \rightarrow \mathcal{O}_{S_{0}} \rightarrow 0
$$

and note that

$$
\mathscr{I}=\mathscr{A}\left(1-\epsilon_{1}\right) \mathscr{A} \cong \mathscr{A}\left(1-\epsilon_{1}\right) \stackrel{\mathbb{L}}{\mathscr{Q}_{\mathscr{A}^{\prime}}}\left(1-\epsilon_{1}\right) \mathscr{A} .
$$

Indeed, it is clear from (38) that $\left(1-\epsilon_{1}\right) \mathscr{A}$ is isomorphic to $\mathscr{A}^{\prime} \epsilon_{2} \oplus \mathscr{A}^{\prime}$ as an $\mathscr{A}^{\prime}$-module (if we remove the first column from (38), we get $\mathscr{A}^{\prime}$; the first column itself just repeats the first column of $\mathscr{A}^{\prime}$, so the corresponding $\mathscr{A}^{\prime}$-module is the projective module corresponding to the first idempotent of $\mathscr{A}^{\prime}$ which is the second idempotent of $\mathscr{A}$ ), hence it is projective and the RHS above is isomorphic to

$$
\mathscr{A}\left(1-\epsilon_{1}\right) \stackrel{\mathbb{L}}{\otimes} \mathscr{A}^{\prime}\left(\mathscr{A}^{\prime} \epsilon_{2} \oplus \mathscr{A}^{\prime}\right) \cong \mathscr{A}\left(1-\epsilon_{1}\right) \epsilon_{2} \oplus \mathscr{A}\left(1-\epsilon_{1}\right)=\mathscr{A} \epsilon_{2} \oplus \mathscr{A}\left(1-\epsilon_{1}\right),
$$

which clearly coincides with $\mathscr{I}$. Now it follows that

$$
\mathscr{I} \stackrel{\mathbb{L}}{\otimes} \mathcal{A}_{S_{0}} \cong \mathscr{A}\left(1-\epsilon_{1}\right) \stackrel{\mathbb{L}}{\otimes} \mathscr{A}^{\prime}\left(1-\epsilon_{1}\right) \mathscr{A}^{\mathbb{L}} \mathscr{A}_{\mathscr{A}} \mathcal{O}_{S_{0}} \cong \mathscr{A}\left(1-\epsilon_{1}\right) \stackrel{\mathbb{L}}{\otimes} \mathscr{A}^{\prime}\left(1-\epsilon_{1}\right) \mathcal{O}_{S_{0}}=0
$$

since $1-\epsilon_{1}$ acts trivially on $\mathcal{O}_{S_{0}}$. Now tensoring (44) with $\mathcal{O}_{S_{0}}$, we conclude that

$$
\mathcal{O}_{S_{0}} \stackrel{\mathbb{L}}{\otimes} \mathcal{O}_{S_{0}} \cong \mathscr{A} \stackrel{\mathbb{L}}{\otimes} \mathscr{A}_{S_{0}} \cong \mathcal{O}_{S_{0}}
$$

This shows that the composition $\mathrm{Li}^{*} \circ \mathrm{i}$ is isomorphic to the identity.

All the other compositions are evident. Indeed, $\mathrm{e}^{!}(\mathrm{i}(M))=M\left(1-\epsilon_{1}\right)=0$ for any object $M \in \mathbf{D}\left(S_{0}\right)$ since, as we already observed, $1-\epsilon_{1}$ acts on all $\mathcal{O}_{S_{0}}$-modules trivially. Analogously,

$$
L i^{*}(\mathrm{e}(M))=M \stackrel{\mathbb{L}}{\otimes} \mathscr{A}^{\prime}\left(1-\epsilon_{1}\right) \mathscr{A} \stackrel{\mathbb{L}}{\otimes} \mathscr{A}_{S_{0}} \cong M \stackrel{\mathbb{L}}{\otimes} \mathscr{A}_{S^{\prime}}\left(1-\epsilon_{1}\right) \mathcal{O}_{S_{0}}=0
$$

and the reason is the same. Finally, by (37),

$$
\mathrm{e}^{!}(\mathrm{e}(M))=M \stackrel{\mathbb{L}}{\otimes} \mathscr{A}^{\prime}\left(1-\epsilon_{1}\right) \mathscr{A}\left(1-\epsilon_{1}\right)=M \stackrel{\mathbb{L}}{\otimes} \mathscr{A}^{\prime} \mathscr{A}^{\prime} \cong M
$$

so the composition $\mathrm{e}^{!} \circ \mathrm{e}$ is the identity.

Now, we can prove that the category $\mathbf{D}(\mathscr{A})$ has a semiorthogonal decomposition. 
Proposition 5.14. There are semiorthogonal decompositions

$$
\mathbf{D}(\mathscr{A})=\left\langle\mathrm{i}\left(\mathbf{D}\left(S_{0}\right)\right), \mathrm{e}\left(\mathbf{D}\left(\mathscr{A}^{\prime}\right)\right)\right\rangle, \quad \mathbf{D}^{b}(\operatorname{coh}(\mathscr{A}))=\left\langle\mathrm{i}\left(\mathbf{D}^{b}\left(\operatorname{coh}\left(S_{0}\right)\right)\right), \mathrm{e}\left(\mathbf{D}^{b}\left(\operatorname{coh}\left(\mathscr{A}^{\prime}\right)\right)\right)\right\rangle .
$$

Proof. First consider the category $\mathbf{D}(\mathscr{A})$. We already know that the functors $\mathrm{i}$ and e are fully faithful and have the left and the right adjoint, respectively. Moreover,

$$
\operatorname{Hom}_{\mathbf{D}(\mathscr{A})}\left(\mathrm{e}\left(M^{\prime}\right), \mathrm{i}\left(M_{0}\right)\right) \cong \operatorname{Hom}_{\left.\mathbf{D}(\mathscr{A})^{\prime}\right)}\left(M^{\prime}, \mathrm{e}^{!}\left(\mathrm{i}\left(M_{0}\right)\right)\right)=0
$$

by (43), hence the subcategories are semiorthogonal. It remains to check that each object $M \in \mathbf{D}(\mathscr{A})$ fits into a triangle with the other vertices in $\mathrm{i}\left(\mathbf{D}\left(S_{0}\right)\right)$ and $\mathrm{e}\left(\mathbf{D}\left(\mathscr{A}^{\prime}\right)\right)$. For this, we tensor the sequence (44) with $M$ to get a distinguished triangle

$$
M \stackrel{\mathbb{L}}{\otimes} \mathscr{A} \rightarrow M \rightarrow M \stackrel{\mathbb{L}}{\otimes} \mathcal{O}_{S_{0}}
$$

Note that the last term is nothing but $\mathrm{i}\left(\mathrm{Li}^{*}(M)\right)$, so it remains to note that

$$
\mathrm{e}\left(\mathrm{e}^{!}(M)\right) \cong\left(M \stackrel{\mathbb{L}}{\otimes} \mathscr{A} \mathscr{A}\left(1-\epsilon_{1}\right)\right) \stackrel{\mathbb{L}}{\otimes} \mathscr{A}^{\prime}\left(1-\epsilon_{1}\right) \mathscr{A} \cong M \stackrel{\mathbb{L}}{\otimes} \mathscr{A} \mathscr{I}
$$

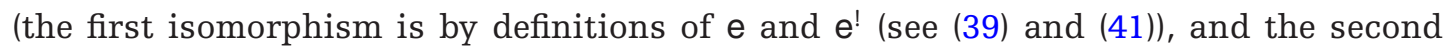
follows from (45)). So, we see that the above triangle can be rewritten as

$$
\mathrm{e}\left(\mathrm{e}^{!}(M)\right) \rightarrow M \rightarrow \mathrm{i}\left(L \mathrm{i}^{*}(M)\right),
$$

which proves the first decomposition.

For the second semiorthogonal decomposition note that we clearly have $\mathrm{i}\left(\mathbf{D}^{b}\left(\operatorname{coh}\left(S_{0}\right)\right)\right) \subset \mathbf{D}^{b}(\operatorname{coh}(\mathscr{A}))$. Further, since $\left(1-\epsilon_{1}\right) \mathscr{A}$ is a projective $\mathscr{A}^{\prime}$-module (see the proof of Proposition 5.13), we deduce that $\mathrm{e}\left(\mathbf{D}^{b}\left(\operatorname{coh}\left(\mathscr{A}^{\prime}\right)\right)\right) \subset \mathbf{D}^{b}(\operatorname{coh}(\mathscr{A}))$. So, it remains to check inclusions for the adjoint functors.

The inclusion $\mathrm{e}^{!}\left(\mathbf{D}^{b}(\operatorname{coh}(\mathscr{A}))\right) \subset \mathbf{D}^{b}\left(\operatorname{coh}\left(\mathscr{A}^{\prime}\right)\right)$ follows immediately from (41). To prove the inclusion for $L^{*}$, consider the triangle (46) with $M \in \mathrm{D}^{b}(\operatorname{coh}(\mathscr{A}))$. Then, from the above it follows that its first vertex is also in $\mathbf{D}^{b}(\operatorname{coh}(\mathscr{A}))$, hence the same is true for the last vertex. It remains to note that $\mathrm{i}\left(L \mathrm{i}^{*}(M)\right) \in \mathbf{D}^{b}(\operatorname{coh}(\mathscr{A}))$ implies $L \mathrm{i}^{*}(M) \in \mathbf{D}^{b}\left(\operatorname{coh}\left(S_{0}\right)\right)$ since the functor $i$ is evidently conservative. 
Iterating the above argument we deduce the following corollary.

Corollary 5.15. There are semiorthogonal decompositions

$$
\begin{aligned}
& \mathbf{D}\left(\mathscr{A}_{S, \mathfrak{r}, n}\right)=\langle\underbrace{\mathbf{D}\left(S_{0}\right), \mathbf{D}\left(S_{0}\right), \ldots, \mathbf{D}\left(S_{0}\right)}_{n \text { components }}\rangle, \\
& \mathbf{D}^{b}\left(\operatorname{coh}\left(\mathscr{A}_{S, \mathfrak{r}, n}\right)\right)=\langle\underbrace{\mathbf{D}^{b}\left(\operatorname{coh}\left(S_{0}\right)\right), \mathbf{D}^{b}\left(\operatorname{coh}\left(S_{0}\right)\right), \ldots, \mathbf{D}^{b}\left(\operatorname{coh}\left(S_{0}\right)\right)}_{n \text { components }}\rangle .
\end{aligned}
$$

Example 5.16. Let $S=\operatorname{Spec} \mathbb{k}[t] / t^{2}, \mathfrak{r}=t \mathbb{k}[t] / t^{2}, n=2$. Then $S_{0}=\operatorname{Spec} \mathbb{k}$, so the semiorthogonal decomposition is just an exceptional pair $\left(E_{1}, E_{2}\right)$ generating the category. The corresponding exceptional objects are

$$
E_{1}=(\mathbb{k} \longleftarrow 0) \text { and } E_{2}=(\mathbb{k} \underset{0}{\stackrel{1}{\longrightarrow}} \mathbb{k}) \text {, }
$$

the simple module of the first vertex and the projective module of the second vertex, respectively. The easiest way to check that the pair is exceptional is by using the projective resolution $0 \rightarrow P_{2} \rightarrow P_{1} \rightarrow E_{1} \rightarrow 0$.

\subsection{Perfect and compact $\mathscr{A}$-modules}

We will say that a complex of $\mathscr{A}$-modules is perfect if it is locally quasiisomorphic to a finite complex of projective $\mathscr{A}$-modules. All perfect complexes form a triangulated subcategory of $\mathbf{D}^{b}(\operatorname{coh}(\mathscr{A}))$ which we denote by $\mathbf{D}^{\text {perf }}(\mathscr{A})$.

Proposition 5.17. If $S_{0}$ is smooth, then $\mathbf{D}^{b}(\operatorname{coh}(\mathscr{A}))=\mathbf{D}^{\text {perf }}(\mathscr{A})$.

Proof. As we already observed, the embedding $\mathbf{D}^{\text {perf }}(\mathscr{A}) \subset \mathbf{D}^{b}(\operatorname{coh}(\mathscr{A}))$ is automatic. For the opposite we use induction on $n$. For $n=1$, the fact is well known. Now assume that $n>1$. Take any $M \in \mathbf{D}^{b}(\operatorname{coh}(\mathscr{A}))$ and consider the triangle (46). In the proof of Proposition 5.14, we checked that $\mathrm{e}^{!}(M) \in \mathbf{D}^{b}\left(\operatorname{coh}\left(\mathscr{A}^{\prime}\right)\right)$ and $L \mathrm{i}^{*}(M) \in \mathbf{D}^{b}\left(\operatorname{coh}\left(S_{0}\right)\right)$. Hence, by induction hypothesis both are perfect. On one hand, as it was observed in Remark 5.10 the functor e takes projective $\mathscr{A}^{\prime}$-modules to projective $\mathscr{A}$-modules, hence e $\left(\mathrm{e}^{!}(M)\right)$ is perfect. On the other hand, it follows that $L^{*}(M)$ is locally quasiisomorphic to a finite complex of free $\mathcal{O}_{S_{0}}$-modules. So, it remains to check that $\mathrm{i}\left(\mathcal{O}_{S_{0}}\right)$ is perfect. 
For this, we note that $\mathcal{O}_{S_{0}} \cong L i^{*}(\mathscr{A})$, hence the triangle (46) for $M=\mathscr{A}$ reads as

$$
\mathrm{e}\left(\mathrm{e}^{!}(\mathscr{A})\right) \rightarrow \mathscr{A} \rightarrow \mathrm{i}\left(\mathcal{O}_{S_{0}}\right)
$$

Its first vertex is perfect by the above argument and its second vertex is evidently perfect. Hence, the third vertex is perfect and we are done.

It is also clear that every perfect complex of $\mathscr{A}$-modules is a compact object of $\mathbf{D}(\mathscr{A})$ (the argument of Neeman [22, Example 1.13] can be easily adjusted to our situation). Therefore, the category $\mathbf{D}(\mathscr{A})$ is compactly generated. Moreover, if $S_{0}$ is smooth, then one can also check that all compact objects are perfect.

Proposition 5.18. If $S_{0}$ is smooth, then $\mathbf{D}(\mathscr{A})^{\mathrm{C}}=\mathrm{D}^{\text {perf }}(\mathscr{A})$.

Proof. We will use induction on $n$. If $n=1$, then $\mathbf{D}(\mathscr{A})=\mathbf{D}(S)=\mathbf{D}\left(S_{0}\right)$ and the statement follows from (5).

Assume $n>1$. Let $M$ be an arbitrary compact object in $\mathbf{D}(\mathscr{A})$. By Lemma 2.10(2), we know that $L \mathrm{i}^{*}(M) \in \mathbf{D}\left(S_{0}\right)^{\mathrm{c}}$ since the functor i commutes with arbitrary direct sums. It follows that $L \mathrm{i}^{*}(M) \in \mathbf{D}^{b}\left(\operatorname{coh}\left(S_{0}\right)\right)$, hence $\mathrm{i}\left(L \mathrm{i}^{*}(M)\right) \in \mathbf{D}^{b}(\operatorname{coh}(\mathscr{A}))$. Thus, by Proposition 5.17,

$$
\mathrm{i}\left(L \mathrm{i}^{*}(M)\right) \in \mathrm{D}^{\text {perf }}(\mathscr{A}) .
$$

In particular, $\mathrm{i}\left(L \mathrm{i}^{*}(M)\right)$ is compact. Using triangle $(46)$, we then conclude that e $\left(\mathrm{e}^{!}(M)\right)$ is compact as well. Further, Lemma 2.10(1) shows that $\mathrm{e}^{!}(M) \in \mathbf{D}\left(\mathscr{A}^{\prime}\right)^{\mathrm{c}}$ since e is fully faithful and commutes with direct sums, and hence by induction assumption, $\mathrm{e}^{!}(M) \in \mathbf{D}\left(\mathscr{A}^{\prime}\right)^{\text {perf }}$. Again, it follows that $\mathrm{e}\left(\mathrm{e}^{!}(M)\right) \in \mathbf{D}^{b}(\operatorname{coh}(\mathscr{A}))$, and hence by Proposition 5.17,

$$
\mathrm{e}\left(\mathrm{e}^{!}(M)\right) \in \mathrm{D}^{\text {perf }}(\mathscr{A})
$$

Looking again at triangle (46) we conclude that $M$ is perfect.

\subsection{DG-enhancement}

We have an analog of Theorem 3.11. 
Theorem 5.19. There is a pseudofunctor $\mathscr{D}: \mathrm{ASch}^{\mathrm{op}} \rightarrow \mathrm{sDG}$ extending the pseudofunctor of Theorem 3.11, such that the diagram

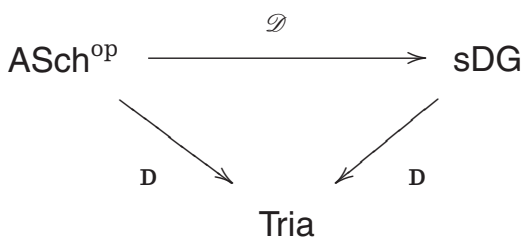

is commutative.

Proof. The proof is analogous to that of Theorem 3.11. We define

$$
\mathscr{D}\left(\mathscr{A}_{S}\right) \subset \text { h-flat-perf }(\mathscr{A}) / \mathrm{h}^{- \text {flat }^{\circ}}{ }^{(\mathscr{A})}
$$

to be a small DG-subcategory such that $\left[\mathscr{D}\left(\mathscr{A}_{S}\right)\right] \cong \mathrm{D}^{\text {perf }}\left(\mathscr{A}_{S}\right)$ and such that, for any morphism of $\mathscr{A}$-spaces $f:\left(T, \mathscr{A}_{T}\right) \rightarrow\left(S, \mathscr{A}_{S}\right)$, we have $f^{*}\left(\mathscr{D}\left(\mathscr{A}_{S}\right)\right) \subset \mathscr{D}\left(\mathscr{A}_{T}\right)$. This gives a DGfunctor $f^{*}: \mathscr{D}\left(\mathscr{A}_{S}\right) \rightarrow \mathscr{D}\left(\mathscr{A}_{T}\right)$. The commutativity of the diagram is proved by the arguments of Theorem 3.11 and compatibility with the pseudofunctor on schemes is by construction.

The following observation is very important.

Theorem 5.20. If the scheme $S_{0}$ is smooth, then the category $\mathscr{D}(\mathscr{A})$ is a smooth DGcategory. If additionally $S_{0}$ is proper, then the category $\mathscr{D}(\mathscr{A})$ is proper.

Proof. We use induction in $n$. The case $n=1$ is evident as $\mathscr{D}(\mathscr{A}) \cong \mathscr{D}(S)$ in this case and $S=S_{0}$ is smooth. So we assume $n>1$. By Proposition 4.10 and Proposition 4.14, the semiorthogonal decomposition of Proposition 5.14 implies that the DG-category $\mathscr{D}(\mathscr{A})$ is quasiequivalent to the gluing

$$
\mathscr{D}(\mathscr{A}) \cong \mathscr{D}\left(S_{0}\right) \times_{\varphi} \mathscr{D}\left(\mathscr{A}^{\prime}\right)
$$

for appropriate bimodule $\varphi$. Moreover, the categories $\mathscr{D}\left(S_{0}\right)$ and $\mathscr{D}\left(\mathscr{A}^{\prime}\right)$ are smooth (the first by smoothness of $S_{0}$ and the second by the induction hypothesis), and so, by Proposition 4.9 , we only have to check that the gluing bimodule $\varphi$ is perfect. 
Note that the functor i $: \mathbf{D}\left(S_{0}\right) \rightarrow \mathbf{D}(\mathscr{A})$ commutes with direct sums, and hence has a right adjoint functor $\mathrm{i}^{!}: \mathbf{D}(\mathscr{A}) \rightarrow \mathbf{D}\left(S_{0}\right)$. By Proposition 3.6, to check that $\varphi$ is perfect it suffices to check that the functor $L \varphi: \mathbf{D}\left(\mathscr{A}^{\prime}\right) \rightarrow \mathbf{D}\left(S_{0}\right)$ preserves compactness. By Proposition 4.6, this functor is isomorphic to the gluing functor i! $\circ$ e : $\mathbf{D}\left(\mathscr{A}^{\prime}\right) \rightarrow \mathbf{D}\left(S_{0}\right)$ of the semiorthogonal decomposition. So, we need to check that the composition $i^{!} \circ$ e preserves compactness.

Since the functor $\mathrm{i}$ is fully faithful and commutes with direct sums, by Lemma 2.10(1) this is equivalent to checking that the functor $\mathrm{i} \circ \mathrm{i}^{!} \circ \mathrm{e}: \mathrm{D}\left(\mathscr{A}^{\prime}\right) \rightarrow \mathbf{D}(\mathscr{A})$ preserves compactness. Note that e preserves compactness by Lemma 2.10(2), since its right

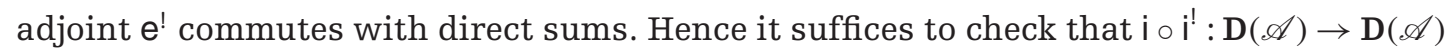
preserves compactness. Since an object in $\mathbf{D}(\mathscr{A})$ is compact if and only if it is bounded and coherent (by Propositions 5.17 and 5.18), and since the latter by definition is equivalent to boundedness and coherence of its image under $\operatorname{Res}_{S}$, it is enough to check that, for any compact $N \in \mathbf{D}(\mathscr{A})^{\mathrm{c}}$, one has $\operatorname{Res}_{S}\left(\mathrm{i}\left(\mathrm{i}^{!}(N)\right)\right) \in \mathbf{D}^{b}(\operatorname{coh}(S))$.

Note that $\operatorname{Res}_{S} \circ \mathbf{i} \circ \mathrm{i}^{!}$is right adjoint to the functor $\mathrm{i} \circ L^{*} \circ \operatorname{LInd}_{S}: \mathbf{D}(S) \rightarrow \mathbf{D}(\mathscr{A})$, which is given by $M \mapsto\left(M \stackrel{\mathbb{L}}{\otimes} \mathcal{O}_{S} \mathscr{A}\right) \stackrel{\mathbb{L}}{\otimes} \mathscr{A}_{\mathscr{O}} \mathcal{O}_{S_{0}} \cong M \stackrel{\mathbb{L}}{\otimes} \mathcal{O}_{S} \mathcal{O}_{S_{0}}$. It follows immediately that the functor $\operatorname{Res}_{S} \circ \mathbf{i} \circ \mathbf{i}^{!}$is isomorphic to

$$
\operatorname{Res}_{S}\left(\mathrm{i}\left(\mathrm{i}^{!}(N)\right)\right)=\mathrm{R} \mathscr{H} \text { om } m_{\mathscr{A}}\left(\mathcal{O}_{S_{0}}, N\right)
$$

It remains to note that, by Proposition 5.17, the sheaf $\mathcal{O}_{S_{0}}$ considered as a right $\mathscr{A}$ module has a finite resolution by locally projective $\mathscr{A}$-modules of finite rank, which means that $\mathrm{R} \mathscr{H} 0 m_{\mathscr{A}}\left(\mathcal{O}_{S_{0}}, N\right)$ is bounded and coherent. And this is precisely what we had to check.

\subsection{The resolution}

Let us consider one very special morphism of $\mathscr{A}$-spaces, namely the morphism of $\mathscr{A}$ spaces

$$
(S, \mathfrak{r}, n) \stackrel{\rho_{S}}{\longrightarrow}(S, 0,1)
$$

induced by the identity morphism id $S: S \rightarrow S$ of the scheme $S$. In the target we just take the same underlying reducible scheme $S$ as in the source, while the defining ideal is taken to be 0 and the width is taken to be 1 . Note that $\mathscr{A}_{S, 0,1}=\mathcal{O}_{S}$ as was observed in Remark 5.2. Consider the corresponding pullback functor $\rho_{S}^{*}: \operatorname{Qcoh}(S) \rightarrow \operatorname{Qcoh}\left(\mathscr{A}_{S}\right)$ and the corresponding pushforward functor $\rho_{S *}: \operatorname{Qcoh}\left(\mathscr{A}_{S}\right) \rightarrow \operatorname{Qcoh}(S)$. By definition (34), we 
have

$$
\rho_{S}^{*}(M)=M \otimes_{\mathcal{O}_{S}}\left(\epsilon_{1} \mathscr{A}\right), \quad \rho_{S *}(N)=N \epsilon_{1}
$$

Lemma 5.21. The functor $\rho_{S}^{*}$ is the left adjoint of $\rho_{S *}$ and $\rho_{S *} \circ \rho_{S}^{*} \cong \mathrm{id}$.

Proof. The adjunction is the particular case of Lemma 5.4. The composition of functors is also easy to compute

$$
\rho_{S *}\left(\rho_{S}^{*}(M)\right)=\left(M \otimes_{\mathcal{O}_{S}}\left(\epsilon_{1} \mathscr{A}\right)\right) \epsilon_{1}=M \otimes_{\mathcal{O}_{S}}\left(\epsilon_{1} \mathscr{A} \epsilon_{1}\right)=M \otimes_{\mathcal{O}_{S}} \mathcal{O}_{S}=M
$$

so $\rho_{S *} \circ \rho_{S}^{*}=\mathrm{id}$.

Example 5.22. Let $S=\operatorname{Spec} \mathbb{k}[t] / t^{2}$. Then $\rho_{S}^{*}(M)=(M \stackrel{t}{\longleftarrow} M / t M)$. Alternatively, it can be written as $\rho_{S}^{*}(M)=M \otimes_{\mathbb{k}[t] / t^{2}} P_{1}$, where $P_{1}=\left(\mathbb{k}[t] / t^{2} \stackrel{t}{\longleftrightarrow} k\right)$ is the projective module of the first vertex of the quiver.

In the same way as general pullback functors do, the functor $\rho_{S}^{*}$ extends to a DG-functor $\rho_{S}^{*}: \mathscr{D}(S) \rightarrow \mathscr{D}\left(\mathscr{A}_{S}\right)$ as well as to a triangulated functor on derived categories $L \rho_{S}^{*}: \mathbf{D}(S) \rightarrow \mathbf{D}\left(\mathscr{A}_{S}\right)$. The functor $\rho_{S *}$ is exact by (47) and so automatically descends to a functor on derived categories $\rho_{S *}: \mathbf{D}\left(\mathscr{A}_{S}\right) \rightarrow \mathbf{D}(S)$. The adjunction between $\rho_{S}^{*}$ and $\rho_{S *}$ induces an adjunction between $L \rho_{S}^{*}$ and $\rho_{S *}$ and isomorphism of Lemma 5.21 gives an isomorphism

$$
\rho_{S *} \circ L \rho_{S}^{*} \cong \operatorname{id}_{\mathbf{D}(S)}
$$

The following result now easily follows.

Theorem 5.23. If the scheme $S_{0}$ is smooth, then

(1) the functor $\rho_{S}^{*}: \mathscr{D}(S) \rightarrow \mathscr{D}\left(\mathscr{A}_{S}\right)$ is a categorical DG-resolution;

(2) the functor $L \rho_{S}^{*}: \mathbf{D}(S) \rightarrow \mathbf{D}\left(\mathscr{A}_{S}\right)$ is a categorical resolution.

In particular, the functor $\rho_{S *}$ takes $\mathbf{D}^{b}\left(\operatorname{coh}\left(\mathscr{A}_{S}\right)\right)$ to $\mathbf{D}^{b}(\operatorname{coh}(S))$. Finally, if $S_{0}$ is proper, then so is the category $\mathscr{D}\left(\mathscr{A}_{S}\right)$.

Proof. The category $\mathscr{D}\left(\mathscr{A}_{S}\right)$ is smooth by Theorem 5.20 . Moreover, by (48) the functor $L \rho_{S}^{*}$ is fully faithful, hence $\rho_{S}^{*}$ is quasi-fully faithful. So, part (1) follows. 
By Proposition 3.13 to deduce part (2) we only have to check that the functor $\rho_{S *}$ takes $\left[\mathscr{D}\left(\mathscr{A}_{S}\right)\right]=\mathbf{D}\left(\mathscr{A}_{S}\right)^{\text {perf }}$ to $\mathbf{D}^{b}(\operatorname{coh}(S))$. By Proposition $5.17, \mathbf{D}\left(\mathscr{A}_{S}\right)^{\text {perf }}=\mathbf{D}^{b}\left(\operatorname{coh}\left(\mathscr{A}_{S}\right)\right)$ and by Lemma 5.8 we have $\rho_{S *}\left(\mathbf{D}^{b}\left(\operatorname{coh}\left(\mathscr{A}_{S}\right)\right) \subset \mathbf{D}^{b}(\operatorname{coh}(S))\right.$, which completes the proof.

Finally, the properness of $\mathscr{D}\left(\mathscr{A}_{S}\right)$ is also proved in Theorem 5.20.

We will also need one more observation. Assume that $f:\left(T, \mathfrak{r}_{T}, n_{T}\right) \rightarrow\left(S, \mathfrak{r}_{S}, n_{S}\right)$ is a morphism of $\mathscr{A}$-spaces. Then, we have a commutative diagram

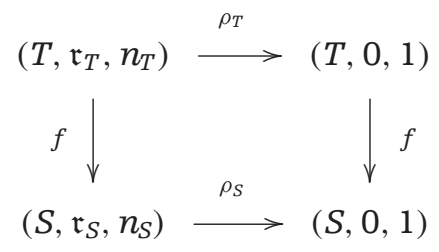

of morphisms of $\mathscr{A}$-spaces which includes the resolution morphisms $\rho_{S}$ and $\rho_{T}$.

Lemma 5.24. There is an isomorphism of DG-functors $f^{*} \rho_{S}^{*} \cong \rho_{T}^{*} f^{*}: \mathscr{D}(S) \rightarrow \mathscr{D}\left(\mathscr{A}_{T}\right)$.

Proof. The proof follows from Lemma 5.5.

\section{A Categorical Resolution of a Scheme}

Let $Y$ be a separated scheme of finite type over $\mathbb{k}$. Let $f: X \rightarrow Y$ be a blowup of a smooth subvariety $Z \subset Y$. We consider an appropriate thickening $S$ of $Z$ in $Y$ and construct a partial categorical DG-resolution of $\mathscr{D}(Y)$ by gluing $\mathscr{D}(\mathscr{A})$ (the DG-resolution of the scheme $S$ constructed in Section 5 ) with $\mathscr{D}(X)$. Then, we use an inductive procedure to combine such partial resolutions into a categorical DG-resolution of $\mathscr{D}(Y)$.

\subsection{Nonrational locus}

For a morphism $f: X \rightarrow Y$ of schemes and a subscheme $S \subset Y$ we denote by $f^{-1}(S)$ the scheme-theoretic preimage of $S$. By definition $f^{-1}(S)$ is the subscheme of $X$ defined by the ideal $f^{-1} I_{S} \cdot \mathcal{O}_{X}$, where $I_{S} \subset \mathcal{O}_{Y}$ is the ideal of $S$.

If $f: X \rightarrow Y$ is a proper morphism of (possibly nonreduced and reducible) schemes, we say that $f$ is birational if, for any irreducible component $Y^{\prime} \subset Y$, the schemetheoretic preimage $X^{\prime}:=f^{-1}\left(Y^{\prime}\right)$ is irreducible and the restriction of $f, X_{\text {red }}^{\prime} \rightarrow Y_{\text {red }}^{\prime}$ is birational. 
Definition 6.1. Let $f: X \rightarrow Y$ be a proper birational morphism. A subscheme $S \subset Y$ is called a nonrational locus of $Y$ with respect to $f$ if the canonical morphism

$$
I_{S} \rightarrow R f_{*}\left(I_{f^{-1}(S)}\right)
$$

is an isomorphism.

Remark 6.2. It is easy to see that if $Y$ is integral and $f: X \rightarrow Y$ is a resolution of singularities, then the empty subscheme is a nonrational locus for $f: X \rightarrow Y$ if and only if $Y$ has rational singularities. Thus, if $S$ is a nonrational locus for $f: X \rightarrow Y$ and $X$ is smooth, then $Y \backslash S$ has rational singularities. This justifies the name.

The following lemma shows that a nonrational locus exists in a pretty large generality.

Lemma 6.3. Assume $f: X \rightarrow Y$ is a blowup of a sheaf of ideals $I$ on $Y$. Then, for $n \gg 0$ the subscheme of $Y$ corresponding to the ideal $I^{n}$ is a nonrational locus with respect to $f$.

Proof. Recall that $X$ is the projective spectrum of the sheaf of graded algebras $\oplus_{k=0}^{\infty} I^{k}$ on $Y$. Further, it is clear that the graded sheaf of modules corresponding to the sheaf $f^{-1} I^{n} \cdot \mathcal{O}_{X}$ is $\oplus_{k=0}^{\infty} I^{k+n}$, which is nothing but the line bundle $\mathcal{O}_{X / Y}(n)$. On the other hand, for $n \gg 0$ we have $R f_{*} \mathcal{O}_{X / Y}(n)=I^{n}$; see, for example, [12, Ex. II.5.9].

Consider the Cartesian square

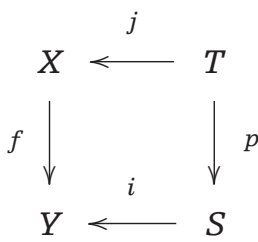

where $S$ is a subscheme of $Y, T=f^{-1}(S)$ is its scheme-theoretic preimage, $i$ and $j$ are the closed embeddings, and $p$ is the restriction of $f$ to $T$.

Lemma 6.4. If $S$ is a nonrational locus for the morphism $f$ and $T=f^{-1}(S)$ is its schemetheoretic preimage, then there is a distinguished triangle in $\mathbf{D}(Y)$

$$
\mathcal{O}_{Y} \rightarrow R f_{*} \mathcal{O}_{X} \oplus i_{*} \mathcal{O}_{S} \rightarrow R f_{*} j_{*} \mathcal{O}_{T}
$$


Proof. We have a commutative diagram

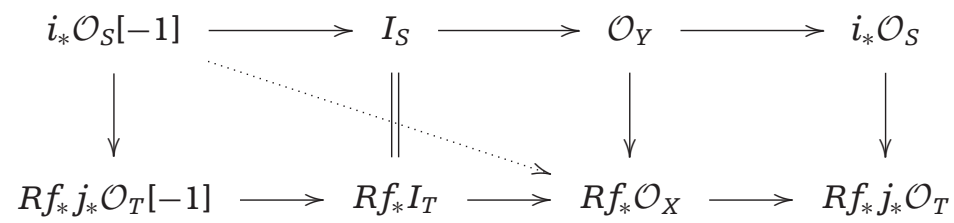

with rows being distinguished triangles. It follows that the dotted arrow is zero. Extending the commutative square marked with $\star$ to the diagram of the octahedron axiom

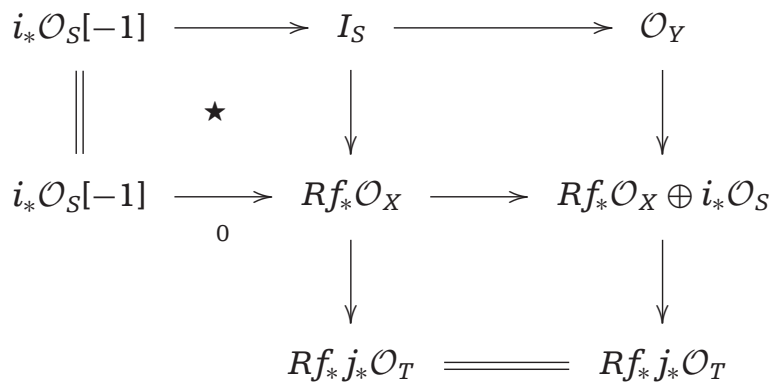

we see the required triangle (50) as its right column.

\subsection{The naive gluing}

Let $f: X \rightarrow Y$ be a blowup with smooth center $Z \subset Y$. Let $I=I_{Z}$ and let $S$ be a nonrational locus for $f$ such that $I_{S}=I^{n}$ (such $n$ exists by Lemma 6.3). Let $T$ be the scheme-theoretic preimage of $S$ and consider the diagram (49). This diagram allows one to define a gluing of $\mathscr{D}(S)$ with $\mathscr{D}(X)$ by using $\mathscr{D}(T)$ to construct the gluing DG-bimodule. To be more precise, we define a DG-bimodule $\varphi_{0} \in \mathscr{D}(X)^{\mathrm{op}} \otimes \mathscr{D}(S)$-dgm by

$$
\varphi_{0}\left(G_{X}, G_{S}\right)=\operatorname{Hom}_{\mathscr{D}(T)}\left(p^{*} G_{S}, j^{*} G_{T}\right)
$$

with the bimodule structure given by DG-functors $j^{*}$ and $p^{*}$, and consider the gluing

$$
\mathscr{D}_{0}:=\mathscr{D}(S) \times_{\varphi_{0}} \mathscr{D}(X) .
$$

The key observation is that $\mathscr{D}_{0}$ provides a partial categorical DG-resolution of $\mathscr{D}(Y)$ as soon as $S$ is a nonrational locus for $f$. Thus, adding the category of a nonrational locus to the category of the blowup allows one to cure nonrationality of the singularity.

Indeed, let us construct a DG-functor from $\mathscr{D}(Y)$ to $\mathscr{D}_{0}$. Let

$$
t=f \circ j=i \circ p: T \rightarrow Y
$$


and note that, for any $F \in \mathscr{D}(Y)$, we have

$$
\varphi_{0}\left(f^{*} F, i^{*} F\right)=\operatorname{Hom}_{\mathscr{D}(T)}\left(p^{*} i^{*} F, j^{*} f^{*} F\right)=\operatorname{Hom}_{\mathscr{D}(T)}\left(t^{*} F, t^{*} F\right) .
$$

This allows one to define

$$
\mu_{F}:=1_{t^{*} F} \in \operatorname{Hom}_{\mathscr{D}(T)}\left(t^{*} F, t^{*} F\right)=\varphi_{0}\left(f^{*} F, i^{*} F\right) .
$$

By construction $\mu_{F}$ is closed of degree zero, so $\left(i^{*} F, f^{*} F, \mu_{F}\right)$ is a well-defined object of the gluing $\mathscr{D}_{0}$. Moreover, it is clear that

$$
\pi_{0}(F):=\left(i^{*} F, f^{*} F, \mu_{F}\right)
$$

is a well-defined DG-functor $\pi_{0}: \mathscr{D}(Y) \rightarrow \mathscr{D}_{0}$. We denote by $\pi_{0}^{*}$ its extension to derived categories:

$$
\pi_{0}^{*}=\operatorname{LInd}_{\pi_{0}}: \mathbf{D}(Y) \rightarrow \mathbf{D}\left(\mathscr{D}_{0}\right) .
$$

Proposition 6.5. The functor $\pi_{0}^{*}: \mathbf{D}(Y) \rightarrow \mathbf{D}\left(\mathscr{D}_{0}\right)$ is fully faithful.

Proof. By Proposition 3.9, it suffices to check that $\pi_{0}$ is quasi-fully faithful. According to Remark 4.1, we have a distinguished triangle

$$
\left.\operatorname{Hom}_{\mathscr{D}_{0}}\left(\pi_{0}(F), \pi_{0}(G)\right) \longrightarrow \begin{array}{c}
\operatorname{Hom}_{\mathscr{D}(S)}\left(i^{*} F, i^{*} G\right) \\
\oplus
\end{array} \begin{array}{c}
\oplus \\
\operatorname{Hom}_{\mathscr{D}(X)}\left(f^{*} F, f^{*} G\right)
\end{array}\right)
$$

Since the DG-bimodule structure on $\varphi_{0}$ is given by the pullback functors $p^{*}$ and $j^{*}$, respectively, and since the elements $\mu_{F}$ and $\mu_{G}$ are given by the units, we can rewrite this triangle as

$$
\operatorname{Hom}_{\mathscr{D}_{0}}\left(\pi_{0}(F), \pi_{0}(G)\right) \longrightarrow \begin{gathered}
\operatorname{Hom}_{\mathscr{D}(S)}\left(i^{*} F, i^{*} G\right) \\
\oplus
\end{gathered} \begin{gathered}
\oplus \\
\operatorname{Hom}_{\mathscr{D}(X)}\left(f^{*} F, f^{*} G\right)
\end{gathered} \stackrel{-p^{*}}{\longrightarrow} \operatorname{Hom}_{\mathscr{D}(T)}\left(t^{*} F, t^{*} G\right)
$$

Note that the composition of the first arrow of this triangle with the action of the DG-functor $\pi_{0}: \operatorname{Hom}_{\mathscr{D}(Y)}(F, G) \rightarrow \operatorname{Hom}_{\mathscr{D}_{0}}\left(\pi_{0}(F), \pi_{0}(G)\right)$ is given by DG-functors $i^{*}$ and $f^{*}$, and so, to prove that $\pi_{0}$ is quasi-fully faithful, it is enough to check that the following 
triangle:

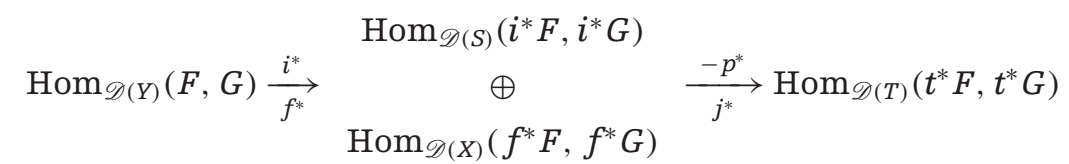

is distinguished.

For this, we identify the vertices of the triangle in terms of $Y$. Indeed, these vertices compute Ext groups in the derived categories $\operatorname{Ext}_{\mathbf{D}(Y)}^{\cdot}(F, G), \operatorname{Ext}_{\mathbf{D}(S)}^{\bullet}\left(L i^{*} F, L i^{*} G\right)$, $\operatorname{Ext}_{\mathbf{D}(X)}^{\bullet}\left(L f^{*} F, L f^{*} G\right)$, and $\operatorname{Ext}_{\mathbf{D}(T)}^{\bullet}\left(L t^{*} F, L t^{*} G\right) \cong \operatorname{Ext}_{\mathbf{D}(T)}^{\cdot}\left(L j^{*} L f^{*} F, L j^{*} L f^{*} G\right)$, respectively. Using the pullback-pushforward adjunctions and the projection formula, we rewrite

$$
\operatorname{Ext}_{\mathbf{D}(S)}^{\bullet}\left(L i^{*} F, L i^{*} G\right) \cong \operatorname{Ext}_{\mathbf{D}(Y)}^{\bullet}\left(F, i_{*} L i^{*} G\right) \cong \operatorname{Ext}_{\mathbf{D}(Y)}^{\bullet}\left(F, G \stackrel{\mathbb{}}{\otimes} i_{*} \mathcal{O}_{S}\right)
$$

for the first summand of the second vertex,

$$
\operatorname{Ext}_{\mathbf{D}(X)}^{\bullet}\left(L f^{*} F, L f^{*} G\right) \cong \operatorname{Ext}_{\mathbf{D}(Y)}^{\bullet}\left(F, R f_{*} L f^{*} G\right) \cong \operatorname{Ext}_{\mathbf{D}(Y)}^{\bullet}\left(F, G \stackrel{\mathbb{}}{\otimes} R f_{*} \mathcal{O}_{X}\right),
$$

for the second summand of the second vertex, and

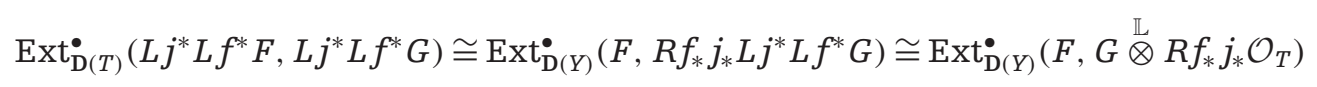

for the third vertex. It is clear that the morphism from the first vertex of (54) to the summands of the second correspond under above isomorphisms to the maps obtained by tensoring morphisms $\mathcal{O}_{Y} \rightarrow i_{*} \mathcal{O}_{S}$ and $\mathcal{O}_{Y} \rightarrow R f_{*} \mathcal{O}_{X}$ with $G$ and applying $\operatorname{Ext}_{\mathbf{D}(Y)}^{\bullet}(F,-)$. Analogously, the morphisms from the summands of the second vertex to the third are obtained by the tensoring morphisms $i_{*} \mathcal{O}_{S} \rightarrow i_{*} R p_{*} \mathcal{O}_{T}=R f_{*} j_{*} \mathcal{O}_{T}$ and $R f_{*} \mathcal{O}_{X} \rightarrow R f_{*} j_{*} \mathcal{O}_{T}$ with $G$ and applying $\operatorname{Ext}_{\mathbf{D}(Y)}^{\bullet}(F,-)$. Therefore the whole triangle (54) is obtained by tensoring with $G$ the triangle

$$
\mathcal{O}_{Y} \rightarrow i_{*} \mathcal{O}_{S} \oplus R f_{*} \mathcal{O}_{X} \rightarrow R f_{*} j_{*} \mathcal{O}_{T}
$$

and applying $\operatorname{Ext}_{\mathbf{D}(Y)}^{\cdot}(F,-)$. The above triangle is distinguished by Lemma 6.4, which implies that the triangle (54) is distinguished as well.

Note that, by Brown representability, the functor $\pi_{0}^{*}$ has a right adjoint which we denote by

$$
\pi_{0 *}=\operatorname{Res}_{\pi_{0}}: \mathbf{D}\left(\mathscr{D}_{0}\right) \rightarrow \mathbf{D}(Y) .
$$

Recall that the derived category $\mathbf{D}\left(\mathscr{D}_{0}\right)$ of the gluing $\mathscr{D}_{0}$ has a semiorthogonal decomposition $\mathbf{D}\left(\mathscr{D}_{0}\right)=\langle\mathbf{D}(S), \mathbf{D}(X)\rangle$; see Section 4.3. In particular, we have embedding functors 
$I_{1}: \mathbf{D}(S) \rightarrow \mathbf{D}\left(\mathscr{D}_{0}\right)$ and $I_{2}: \mathbf{D}(X) \rightarrow \mathbf{D}\left(\mathscr{D}_{0}\right)$ commuting with arbitrary direct sums. We will implicitly identify the categories $\mathbf{D}(S)$ and $\mathbf{D}(X)$ with their images in $\mathbf{D}\left(\mathscr{D}_{0}\right)$.

Proposition 6.6. The functor $\pi_{0 *}$ restricted to $\mathbf{D}(S) \subset \mathbf{D}\left(\mathscr{D}_{0}\right)$ is isomorphic to the pushforward $i_{*}: \mathbf{D}(S) \rightarrow \mathbf{D}(Y)$. The functor $\pi_{0 *}$ restricted to $\mathbf{D}(X) \subset \mathbf{D}\left(\mathscr{D}_{0}\right)$ is isomorphic to the composition $R f_{*}\left(-\stackrel{\mathbb{L}}{\otimes} I_{T}\right): \mathbf{D}(X) \rightarrow \mathbf{D}(Y)$. In particular, $\pi_{0 *}$ takes both $\mathbf{D}^{b}(\operatorname{coh}(S))$ and $\mathbf{D}^{b}(\operatorname{coh}(X))$ to $\mathbf{D}^{b}(\operatorname{coh}(Y))$.

Proof. The functor $\pi_{0 *}=\operatorname{Res}_{\pi_{0}}$ commutes with arbitrary direct sums by Proposition 3.9. The same is true for the embedding functors $\mathbf{D}(S) \rightarrow \mathbf{D}\left(\mathscr{D}_{0}\right)$ and $\mathbf{D}(X) \rightarrow \mathbf{D}\left(\mathscr{D}_{0}\right)$. Hence, the restrictions of the functor $\pi_{0 *}$ onto $\mathbf{D}(S)$ and $\mathbf{D}(X)$ commute with arbitrary direct sums. By [27, Theorem 7.2.], it is enough to prove the claim only for objects of subcategories $[\mathscr{D}(S)] \subset \mathbf{D}(S)$ and $[\mathscr{D}(X)] \subset \mathbf{D}(X)$, respectively.

So, take arbitrary $F \in \mathscr{D}(Y)$ and $G_{S} \in \mathscr{D}(S)$. Since $\pi_{0 *}$ is the right adjoint of $\pi_{0}^{*}$ which coincides with $\pi_{0}$ on $\mathscr{D}(Y)$, we have $\operatorname{Hom}_{\mathbf{D}(Y)}\left(F, \pi_{0 *}\left(G_{S}, 0,0\right)\right) \cong$ $\operatorname{Hom}_{\mathbf{D}\left(\mathscr{D}_{0}\right)}\left(\pi_{0} F,\left(G_{S}, 0,0\right)\right)$, which is just the cohomology of the complex

$$
\operatorname{Hom}_{\mathscr{D}_{0}}\left(\pi_{0}(F),\left(G_{S}, 0,0\right)\right)=\operatorname{Hom}_{\mathscr{D}_{0}}\left(\left(i^{*} F, f^{*} F, \mu_{F}\right),\left(G_{S}, 0,0\right)\right)=\operatorname{Hom}_{\mathscr{D}(S)}\left(i^{*} F, G_{S}\right),
$$

which is nothing but $\operatorname{Hom}_{\mathbf{D}(S)}\left(L i^{*} F, G_{S}\right) \cong \operatorname{Hom}_{\mathbf{D}(Y)}\left(F, i_{*} G_{S}\right)$, which proves the first claim.

Now let $G_{X} \in \mathscr{D}(X)$. Then $\operatorname{Hom}_{\mathbf{D}(Y)}\left(F, \pi_{0 *}\left(0, G_{X}, 0\right)\right) \cong \operatorname{Hom}_{\mathbf{D}\left(\mathscr{D}_{0}\right)}\left(\pi_{0} F,\left(0, G_{X}, 0\right)\right)$, which is just the cohomology of the complex $\operatorname{Hom}_{\mathscr{D}_{0}}\left(\pi_{0}(F),\left(0, G_{X}, 0\right)\right)$, which by Remark 4.1 fits into a distinguished triangle

$$
\operatorname{Hom}_{\mathscr{D}_{0}}\left(\pi_{0}(F),\left(0, G_{X}, 0\right)\right) \longrightarrow \operatorname{Hom}_{\mathscr{D}(X)}\left(f^{*} F, G_{X}\right) \stackrel{\mu_{F}}{\longrightarrow} \varphi_{0}\left(G_{X}, i^{*} F\right) .
$$

Further, the argument goes along the lines of that of Proposition 6.5. First, we rewrite the triangle as

$$
\operatorname{Hom}_{\mathscr{D}_{0}}\left(\pi_{0}(F),\left(0, G_{X}, 0\right)\right) \rightarrow \operatorname{Hom}_{\mathscr{D}(X)}\left(f^{*} F, G_{X}\right) \stackrel{j^{*}}{\rightarrow} \operatorname{Hom}_{\mathscr{D}(T)}\left(j^{*} f^{*} F, j^{*} G_{X}\right) .
$$

Further, we identify the second vertex with $\operatorname{Ext}_{\mathbf{D}(X)}^{\bullet}\left(L f^{*} F, G_{X}\right) \cong \operatorname{Ext}_{\mathbf{D}(Y)}^{\bullet}\left(F, R f_{*} G_{X}\right)$, the third vertex with

$$
\operatorname{Ext}_{\mathbf{D}(T)}^{\bullet}\left(L j^{*} L f^{*} F, L j^{*} G_{X}\right) \cong \operatorname{Ext}_{\mathbf{D}(Y)}^{\bullet}\left(F, R f_{*} j_{*} L j^{*} G_{X}\right) \cong \operatorname{Ext}_{\mathbf{D}(Y)}^{\bullet}\left(F, R f_{*}\left(G_{X} \stackrel{\mathbb{L}}{\otimes} j_{*} \mathcal{O}_{T}\right)\right),
$$

and the map between them with the map obtained by tensoring morphism $\mathcal{O}_{X} \rightarrow j_{*} \mathcal{O}_{T}$ with $G_{X}$ and applying $\operatorname{Ext}_{\mathbf{D}(Y)}^{\bullet}\left(F, R f_{*}(-)\right)$. Looking at the triangle

$$
I_{T} \rightarrow \mathcal{O}_{X} \rightarrow j_{*} \mathcal{O}_{T}
$$


we see that the first term of (55) is quasiisomorphic to $\operatorname{Ext}_{\mathbf{D}(Y)}^{\bullet}\left(F, R f_{*}\left(G_{X} \stackrel{\mathbb{L}}{\otimes} I_{T}\right)\right)$, which proves the second claim of the Proposition.

For the last claim note that the functors $i_{*}$ and $R f_{*}$ preserve boundedness and coherence since the morphisms $i$ and $f$ are proper, and the same is true for the functor $-\stackrel{\mathbb{L}}{\otimes} I_{T}$ because $I_{T} \cong \mathcal{O}_{X / Y}(n)$ is a line bundle.

We know by Proposition 6.5 that $\mathscr{D}_{0}$ is a partial categorical DG-resolution of $\mathscr{D}(Y)$. It is not a resolution yet since both components $\mathscr{D}(S)$ and $\mathscr{D}(X)$ of $\mathscr{D} 0$ are not smooth in general. Of course we can resolve $\mathscr{D}(S)$ by appropriate Auslander algebra $\mathscr{A}_{S}$ as discussed in Section 5 and we can apply the induction to resolve $\mathscr{D}(X)$ by appropriate smooth DG-category $\mathscr{D}^{\prime}$. Then, we can apply the regluing procedure to obtain a (partial) resolution of $\mathscr{D}(Y)$ by $\mathscr{D}\left(\mathscr{A}_{S}\right) \times_{\tilde{\varphi}_{0}} \mathscr{D}^{\prime}$, and to check that it is smooth, we will have to check that the induced bimodule $\tilde{\varphi}_{0}$ is perfect. It turns out, however, that this is not the case. The reason for this is that the pullback functor $L \rho_{S}^{*}: \mathbf{D}(S) \rightarrow \mathbf{D}\left(\mathscr{A}_{S}\right)$ does not preserve boundedness (for details one can see the proof of Theorem 6.12). So, to get rid of this problem, we replace the naive gluing $\mathscr{D}_{0}=\mathscr{D}(S) \times_{\varphi_{0}} \mathscr{D}(X)$ with the gluing of $\mathscr{D}\left(\mathscr{A}_{S}\right)$ and $\mathscr{D}(X)$, which is not the regluing of $\mathscr{D}_{0}$ (see Remark 6.10).

\subsection{The gluing which works}

Again, let $f: X \rightarrow Y$ be a blowup with smooth center $Z \subset Y, I=I_{Z}$, and let $S$ be a nonrational locus for $f$ such that $I_{S}=I^{n}$.

Consider also the ideal $\mathcal{J}=f^{-1} I \cdot \mathcal{O}_{X}$. Then, by definition of the blowup this is an invertible sheaf of ideals, $\mathcal{J}=\mathcal{O}_{X / Y}(1)$, and $I_{T}=\mathcal{J}^{n}$, where $T=f^{-1}(S)$. We also put $\mathfrak{r}_{T}=\mathcal{J} / \mathcal{J}^{n}$ and $n_{T}=n_{S}=n$. Then the morphism $p: T \rightarrow S$ gives a morphism $\left(T, \mathfrak{r}_{T}, n\right) \rightarrow$ $\left(S, \mathfrak{r}_{S}, n\right)$ of $\mathscr{A}$-spaces, which we denote by $\bar{p}$. So, we have a commutative diagram

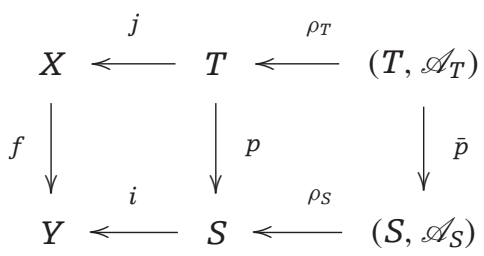

of $\mathscr{A}$-spaces. Now, we replace $\mathscr{D}(S)$ by $\mathscr{D}\left(\mathscr{A}_{S}\right)$ and $\mathscr{D}(T)$ by $\mathscr{D}\left(\mathscr{A}_{T}\right)$ in the construction of the previous section and show that the corresponding gluing works. 
Explicitly, we define the gluing DG-bimodule $\varphi \in \mathscr{D}(X)^{\mathrm{op}} \otimes \mathscr{D}\left(\mathscr{A}_{S}\right)$-dgm as

$$
\varphi\left(G_{X}, G_{S}\right):=\operatorname{Hom}_{\mathscr{D}\left(\mathscr{A}_{T}\right)}\left(\bar{p}^{*} G_{S}, \rho_{T}^{*} j^{*} G_{X}\right)
$$

for all $G_{S} \in \mathscr{D}\left(\mathscr{A}_{S}\right), G_{X} \in \mathscr{D}(X)$. Consider DG-functor $\tau_{1}:=\rho_{S}: \mathscr{D}(S) \rightarrow \mathscr{D}\left(\mathscr{A}_{S}\right)$ defined in Section 5.7 and the identity DG-functor $\tau_{2}:=\mathrm{id}: \mathscr{D}(X) \rightarrow \mathscr{D}(X)$.

Lemma 6.7. The bimodules $\varphi_{0} \in \mathscr{D}(X)^{\mathrm{op}} \otimes \mathscr{D}(S)$-dgm and $\varphi \in \mathscr{D}(X)^{\mathrm{op}} \otimes \mathscr{D}\left(\mathscr{A}_{S}\right)$-dgm are compatible in the sense of Section 4.6.

Proof. Indeed,

$$
\begin{aligned}
\varphi\left(G_{X}, \rho_{S}^{*} G_{S}\right) & =\operatorname{Hom}_{\mathscr{D}\left(\mathscr{A}_{T}\right)}\left(\bar{p}^{*} \rho_{S}^{*} G_{S}, \rho_{T}^{*} j^{*} G_{X}\right) \cong \operatorname{Hom}_{\mathscr{D}\left(\mathscr{A}_{T}\right)}\left(\rho_{T}^{*} p^{*} G_{S}, \rho_{T}^{*} j^{*} G_{X}\right) \\
& \cong \operatorname{Hom}_{\mathscr{D}(T)}\left(p^{*} G_{S}, j^{*} G_{X}\right)=\varphi_{0}\left(G_{X}, G_{S}\right),
\end{aligned}
$$

the first is the definition of $\varphi$, the second is the commutativity of the right square of (56), the third is quasi full-faithfulness of $\rho_{T}^{*}$, and the fourth is the definition of $\varphi_{0}$.

Consider the gluing DG-category

$$
\mathscr{D}=\mathscr{D}\left(\mathscr{A}_{S}\right) \times_{\varphi} \mathscr{D}(X) .
$$

By Proposition 4.11, we have a quasi-fully faithful DG-functor $\tau: \mathscr{D}_{0} \rightarrow \mathscr{D}$ which takes $\left(G_{S}, G_{X}, \mu\right)$ to $\left(\rho_{S}^{*}\left(G_{S}\right), G_{X}, C(\mu)\right)$, where $c$ is the quasiisomorphism of Lemma 6.7. Composing it with the DG-functor $\pi_{0}: \mathscr{D}(Y) \rightarrow \mathscr{D}_{0}$, we obtain a DG-functor

$$
\pi=\tau \circ \pi_{0}: \mathscr{D}(Y) \rightarrow \mathscr{D}, \quad \pi(F)=\left(\rho_{S}^{*} i^{*} F, f^{*} F, C\left(\mu_{F}\right)\right) .
$$

Theorem 6.8. Let $f: X \rightarrow Y$ be the blowing up of an ideal $I$ and $S$ be the subscheme of $Y$ defined by a power of the ideal $I$ which is a nonrational locus for $f$. Then, the DG-functor $\pi: \mathscr{D}(Y) \rightarrow \mathscr{D}=\mathscr{D}\left(\mathscr{A}_{S}\right) \times_{\varphi} \mathscr{D}(X)$ is a partial categorical DG-resolution of singularities. Moreover, the functor $\pi_{*}=\operatorname{Res}_{\pi}: \mathbf{D}(\mathscr{D}) \rightarrow \mathbf{D}(Y)$ takes both $\mathbf{D}^{b}\left(\operatorname{coh}\left(\mathscr{A}_{S}\right)\right)$ and $\mathbf{D}^{b}(\operatorname{coh}(X))$ to $\mathbf{D}^{b}(\operatorname{coh}(Y))$.

Proof. The DG-functor $\pi$ is a composition of the DG-functor $\pi_{0}$ which is quasi-fully faithful by Proposition 6.5 and of the DG-functor $\tau: \mathscr{D}_{0} \rightarrow \mathscr{D}$ which is quasi-fully faithful by Proposition 4.11. Hence, $\pi$ is quasi-fully faithful, and so it is a partial categorical DG-resolution. Further, the functor $\operatorname{Res}_{\pi}$ is isomorphic to the composition $\operatorname{Res}_{\pi_{0}} \circ \operatorname{Res}_{\tau}$. 
By Proposition 4.11(d), the functor $\operatorname{Res}_{\tau}$ on the component $\mathbf{D}\left(\mathscr{A}_{S}\right)$ equals $\rho_{S *}$, hence takes $\mathbf{D}^{b}\left(\operatorname{coh}\left(\mathscr{A}_{S}\right)\right)$ to $\mathbf{D}^{b}(\operatorname{coh}(S))$ by Theorem 5.23. Similarly, by Proposition $4.11(\mathrm{e})$ (see also Remark 4.12) the functor $\operatorname{Res}_{\tau}$ on the component $\mathbf{D}(X)$ is the identity. By Proposition 6.6, the functor $\operatorname{Res}_{\pi_{0}}$ takes both $\mathbf{D}^{b}(\operatorname{coh}(S))$ and $\mathbf{D}^{b}(\operatorname{coh}(X))$ to $\mathbf{D}^{b}(\operatorname{coh}(Y))$, so the claim follows.

The following property of the gluing bimodule $\varphi$ is crucial.

Lemma 6.9. The functor $L \varphi: \mathbf{D}(X) \rightarrow \mathbf{D}\left(\mathscr{A}_{S}\right)$ takes $\mathbf{D}^{b}(\operatorname{coh}(X))$ to $\mathbf{D}^{b}\left(\operatorname{coh}\left(\mathscr{A}_{S}\right)\right)$.

Proof. By definition (13), the functor $L \varphi$ is the functor of derived tensor product over $\mathscr{D}(X)$ with $\varphi$. On the other hand, by (57) we have $\varphi=\rho_{T}^{*} j^{*} \mathscr{D}\left(\mathscr{A}_{T}\right)_{\bar{p}^{*}}$, the restriction of the diagonal bimodule over $\mathscr{D}\left(\mathscr{A}_{T}\right)$. Hence

$$
L \varphi(M)=M \stackrel{\mathbb{L}}{\otimes} \mathscr{D}(X) \varphi=M \stackrel{\mathbb{L}}{\otimes} \mathscr{D}(X) \rho_{T}^{*} j^{*} \mathscr{D}\left(\mathscr{A}_{T}\right)_{\bar{p}^{*}}=\operatorname{Res}_{\bar{p}^{*}}\left(\operatorname{LInd}_{\rho_{T}^{*} j^{*}}(M)\right) .
$$

The derived induction functor $\operatorname{LInd}_{\rho_{T}^{*} j^{*}}$ is isomorphic to the composition of the derived pullback functors $L \rho_{T}^{*} L j^{*}$ by Theorem 5.19. The restriction functor $\operatorname{Res}_{\bar{p}^{*}}$ is right adjoint to the derived induction functor LInd $\bar{p}_{\bar{p}^{*}}$, which by the same theorem is isomorphic to $L \bar{p}^{*}$. Hence $\operatorname{Res}_{\bar{p}^{*}} \cong R \bar{p}_{*}$. Thus,

$$
L \varphi \cong R \bar{p}_{*} \circ L \rho_{T}^{*} \circ L j^{*}: \mathbf{D}(X) \rightarrow \mathbf{D}\left(\mathscr{A}_{S}\right)
$$

The composition of functors $L \rho_{T}^{*} \circ L j^{*}$ is given by $F \mapsto F \stackrel{\mathbb{L}}{\otimes} \mathcal{O}_{X} \mathscr{A}_{T}$. Note that all ideals $\mathcal{J}^{k} \subset \mathcal{O}_{X}$ are invertible, hence all the components $\mathcal{J}^{k} / \mathcal{J}^{l}$ of the sheaf of algebras $\mathscr{A}_{T}$ are perfect $\mathcal{O}_{X}$-modules. Therefore, the functor $L \rho_{T}^{*} \circ L j^{*}$ preserves both boundedness and coherence of sheaves. On the other hand, the functor $R \bar{p}_{*}$ preserves boundedness and coherence by Lemma 5.8. The lemma follows.

Remark 6.10. Now, it is already clear that the gluing $\mathscr{D}$ is not the regluing of $\mathscr{D}_{0}$. Indeed, if it were so, then we would have an isomorphism $L \varphi \cong L \rho_{S}^{*} \circ L \varphi_{0}$. But it is easy to find an object $M \in \mathbf{D}^{b}(\operatorname{coh}(X))$ such that $L \varphi_{0}(M)$ is not perfect and its pullback under $L \rho_{S}^{*}$ is unbounded.

\subsection{The inductive construction of a categorical DG-resolution}

Now, finally we are ready to prove the main result of the paper, Theorem 1.4. 
We start with a separated scheme $Y$ of finite type over a field $\mathbb{k}$ of characteristic 0 [5, Theorem 1.1]. By [4, Theorem 1.6], there exists a chain

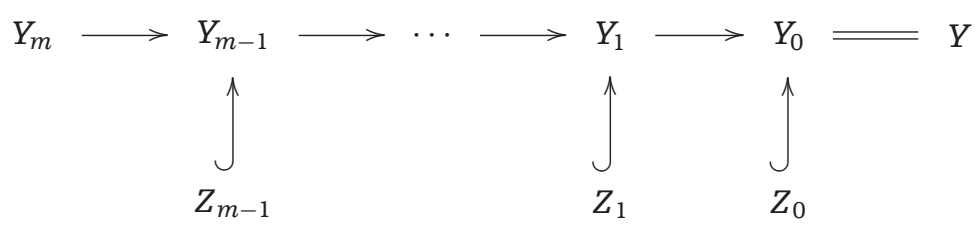

of blowups with smooth centers $Z_{i}$ such that $\left(Y_{m}\right)_{\text {red }}$ is smooth. Indeed, the following lemma shows that the usual resolution of the reduced part of $Y$ by a chain of smooth blowups does the job.

Lemma 6.11. Let $Y$ be a possibly nonreduced scheme and $Z \subset Y_{\text {red }}$ be a smooth subscheme of its reduced part. Then $\left(\mathrm{BL}_{Z} Y\right)_{\text {red }}=\mathrm{BL}_{Z}\left(Y_{\text {red }}\right)$, the reduced part of the blowup equals the blowup of the reduced part.

Proof. The claim is local with respect to $Y$, so we can assume that $Y=\operatorname{Spec} R$ is affine. Let $I \subset R$ be the ideal of $Z$ and let $\mathfrak{r} \subset R$ be the nilpotent radical of $R$. By assumption $\mathfrak{r} \subset I$. By definition of a blowup we have $\mathrm{BL}_{Z} Y=\operatorname{Proj}\left(\oplus I^{k}\right)$. On the other hand, the ideal of $Z$ on $Y_{\text {red }}=\operatorname{Spec}(R / \mathfrak{r})$ is $I / \mathfrak{r}$, hence $\mathrm{BL}_{Z}\left(Y_{\text {red }}\right)=\operatorname{Proj}\left(\oplus I^{k} /\left(I^{k} \cap \mathfrak{r}\right)\right)$. The natural epimorphism of graded algebras

$$
\oplus I^{k} \rightarrow \oplus I^{k} /\left(I^{k} \cap \mathfrak{r}\right)
$$

shows that the blowup $\mathrm{BL}_{Z}\left(Y_{\text {red }}\right)$ is a closed reduced subscheme of $\mathrm{BL}_{Z}(Y)$. Its ideal is given by $\oplus\left(I^{k} \cap \mathfrak{r}\right)$, hence is nilpotent. But a reduced subscheme with nilpotent ideal is nothing but the reduced part of the scheme, hence the claim.

As we already know by Theorem 6.8 one can construct a partial categorical DGresolution of $\mathscr{D}(Y)$ by appropriate gluing of $\mathscr{D}\left(Y_{1}\right)$ with $\mathscr{D}\left(\mathscr{A}_{S}\right)$, where $S$ is an appropriate thickening of $Z_{0}$. Moreover, the component $\mathscr{D}\left(\mathscr{A}_{S}\right)$ of this gluing is smooth by Theorem 5.23 and one can also check that the gluing bimodule is perfect. So, all nonsmoothness of the gluing comes from nonsmoothness of the scheme $Y_{1}$. Thus, to obtain a resolution, we only have to replace (by using the regluing procedure) the component $\mathscr{D}\left(Y_{1}\right)$ by its categorical resolution. This shows that one can use induction to construct the resolution. In fact, it turns out that technically it is much more convenient to include in the induction hypotheses some properties of the resolution as well. So, we state the following theorem. 
Theorem 6.12. There is a small pretriangulated DG-category $\mathscr{D}$ glued from several copies of $\mathscr{D}\left(Y_{m}\right)$ and several copies of $\mathscr{D}\left(Z_{i}\right)$ for $0 \leq i \leq m-1$, and a DG-functor $\pi$ : $\mathscr{D}(Y) \rightarrow \mathscr{D}$ such that

(1) $\mathscr{D}$ is a categorical DG-resolution of $\mathscr{D}(Y)$;

(2) the functor $\pi_{*}=\operatorname{Res}_{\pi}: \mathbf{D}(\mathscr{D}) \rightarrow \mathbf{D}(Y)$ takes $[\mathscr{D}]=\mathbf{D}(\mathscr{D})^{\mathrm{c}}$ to $\mathbf{D}^{b}(\operatorname{coh}(Y))$;

(3) if $Y$ is proper, then so is $\mathscr{D}$.

Proof. We fix a chain of blowups (59) and use induction on $m$. If $m=0$, then the scheme $Y_{\text {red }}$ is smooth, so $\mathscr{D}=\mathscr{D}\left(\mathscr{A}_{Y}\right)$ with $\pi=\rho_{Y}$ provide a categorical DG-resolution of $\mathscr{D}(Y)$ by Theorem 5.23 and, moreover, the properties (2) and (3) are satisfied.

Assume that $m>0$. In this case, we let $X=Y_{1}, Z=Z_{0}$ and denote the blowup map by $f: X \rightarrow Y$. Since $f$ is a blowup of a subscheme $Z \subset Y$, by Lemma 6.3 an appropriate infinitesimal neighborhood $S$ of $Z$ (i.e., the subscheme given by the ideal $I_{Z}^{n}$ for some $n$, where $I_{Z} \subset \mathcal{O}_{Y}$ is the ideal of $Z$ ) is a nonrational locus for $f$. Therefore, Theorem 6.8 applies and we have a partial categorical DG-resolution which we denote here by

$$
\pi_{1}: \mathscr{D}(Y) \rightarrow \mathscr{D}\left(\mathscr{A}_{S}\right) \times_{\varphi} \mathscr{D}(X)
$$

with bimodule $\varphi$ defined by (57). Since the scheme $X=Y_{1}$ can be resolved by $m-1$ smooth blowups, the induction applies; hence we have a categorical DG-resolution

$$
\tau_{2}: \mathscr{D}(X) \rightarrow \mathscr{D}_{2}
$$

Taking $\tau_{1}=\mathrm{id}: \mathscr{D}\left(\mathscr{A}_{S}\right) \rightarrow \mathscr{D}\left(\mathscr{A}_{S}\right)$ and applying the regluing procedure (see Section 4.6), we obtain a partial categorical DG-resolution

$$
\tau: \mathscr{D}\left(\mathscr{A}_{S}\right) \times_{\varphi} \mathscr{D}(X) \rightarrow \mathscr{D}\left(\mathscr{A}_{S}\right) \times_{\tilde{\varphi}} \mathscr{D}_{2}
$$

for appropriate bimodule $\tilde{\varphi}$. By Lemma 3.12, the composition

$$
\pi=\tau \circ \pi_{1}: \mathscr{D}(Y) \rightarrow \mathscr{D}:=\mathscr{D}\left(\mathscr{A}_{S}\right) \times_{\tilde{\varphi}} \mathscr{D}_{2}
$$

is a partial categorical DG-resolution, so the only thing to check for part (1) is that the category $\mathscr{D}$ is smooth.

For this, we note that $\mathscr{D}_{2}$ is smooth by part (1) of the induction hypothesis and $\mathscr{D}\left(\mathscr{A}_{S}\right)$ is smooth by Theorem 5.23. So, by Proposition 4.9 it remains to check that the bimodule $\tilde{\varphi}$ is perfect. For this, we use Proposition 3.6. Since $\mathscr{D}_{2}$ is smooth, it suffices 
to check that the functor $L \tilde{\varphi}: \mathbf{D}\left(\mathscr{D}_{2}\right) \rightarrow \mathbf{D}\left(\mathscr{A}_{S}\right)$ induced by the bimodule $\tilde{\varphi}$ takes [ $\mathscr{D}_{2}$ ] to $\mathbf{D}\left(\mathscr{A}_{S}\right)^{\mathrm{c}}$, which by Propositions 5.18 and 5.17 is $\mathbf{D}^{b}\left(\operatorname{coh}\left(\mathscr{A}_{S}\right)\right)$. For this, we note that, by Proposition 4.13, this functor is isomorphic to the composition

$$
\mathbf{D}\left(\mathscr{D}_{2}\right) \stackrel{\operatorname{Res}_{\tau_{2}}}{\longrightarrow} \mathbf{D}(X) \stackrel{L \varphi}{\longrightarrow} \mathbf{D}\left(\mathscr{A}_{S}\right)
$$

The functor $\operatorname{Res}_{\tau_{2}}$ takes $\left[\mathscr{D}_{2}\right]$ to $\mathbf{D}^{b}(\operatorname{coh}(X))$ by part (2) of the induction hypothesis and the functor $L \varphi$ takes $\mathbf{D}^{b}(\operatorname{coh}(X))$ to $\mathbf{D}^{b}\left(\operatorname{coh}\left(\mathscr{A}_{S}\right)\right)$ by Lemma 6.9. Thus, we have proved part (1). Further, we have to check that the pushforward functor $\operatorname{Res}_{\pi}: \mathbf{D}(\mathscr{D}) \rightarrow \mathbf{D}(Y)$ takes $[\mathscr{D}]$ to $\mathbf{D}^{b}(\operatorname{coh}(Y))$. Since each object of $[\mathscr{D}]$ sits in a triangle with the other vertices in $\mathscr{D}\left(\mathscr{A}_{S}\right)$ and $\left[\mathscr{D}_{2}\right]$, it suffices to check the statement for those. Let $F \in\left[\mathscr{D}\left(\mathscr{A}_{S}\right)\right]$. By Proposition 4.13(b), we have $\operatorname{Res}_{\tau}(F)=\operatorname{Res}_{\tau_{1}}(F)=F$ since $\tau_{1}=\mathrm{id}$, hence

$$
\operatorname{Res}_{\pi}(F)=\operatorname{Res}_{\pi_{1}}\left(\operatorname{Res}_{\tau}(F)\right)=\operatorname{Res}_{\pi_{1}}(F) \in \mathbf{D}^{b}(\operatorname{coh}(Y))
$$

by Proposition 6.6. On the other hand, let $F \in\left[\mathscr{D}_{2}\right]$. Then, again by Proposition 4.13(b), we have $\operatorname{Res}_{\tau}(F)=\operatorname{Res}_{\tau_{2}}(F)$ and this is in $\mathbf{D}^{b}(\operatorname{coh}(X))$ by the induction hypothesis. Then $\operatorname{Res}_{\pi}(F)=\operatorname{Res}_{\pi_{1}}\left(\operatorname{Res}_{\tau_{2}}(F)\right)$ is in $\mathbf{D}^{b}(\operatorname{coh}(Y))$ again by Proposition 6.6. This completes part (2).

Finally, we have to check that $\mathscr{D}$ is proper as soon as $Y$ is. For this, we note that the first center $Z_{0}$ and the first blowup $X=Y_{1}$ are both proper. Hence, by induction hypothesis the resolution $\mathscr{D}_{2}$ of $\mathscr{D}(X)$ is proper. On the other hand, the category $\mathscr{D}\left(\mathscr{A}_{S}\right)$ is proper by Theorem 5.23. So, as we have already seen that the gluing bimodule $\tilde{\varphi}$ is perfect, Proposition 4.9 applies and we conclude that $\mathscr{D}$ is proper.

Now, we are ready to prove Theorem 1.4. Indeed, we take $\mathscr{T}=\mathbf{D}(\mathscr{D})$ and apply Theorem 6.12 together with Proposition 3.13. The semiorthogonal decompositions of $\mathscr{T}$ and $\mathscr{T}^{\mathrm{c}}$ are given by (iterations of) Corollary 4.5 and Proposition 4.6:

$$
\begin{aligned}
\mathbf{D}(\mathscr{D})= & \langle\underbrace{\mathbf{D}\left(Z_{0}\right), \ldots, \mathbf{D}\left(Z_{0}\right)}_{n_{0} \text { times }}, \ldots, \underbrace{\mathbf{D}\left(Z_{m-1}\right), \ldots, \mathbf{D}\left(Z_{m-1}\right)}_{n_{m-1} \text { times }}, \underbrace{\mathbf{D}\left(\left(Y_{m}\right)_{\text {red }}\right), \ldots, \mathbf{D}\left(\left(Y_{m}\right)_{\text {red }}\right)}_{n_{m} \text { times }}\rangle, \\
\mathbf{D}(\mathscr{D})^{\mathrm{C}}= & \langle\underbrace{\mathbf{D}^{b}\left(\operatorname{coh}\left(Z_{0}\right)\right), \ldots, \mathbf{D}^{b}\left(\operatorname{coh}\left(Z_{0}\right)\right)}_{n_{0} \text { times }}, \ldots, \\
& \underbrace{\mathbf{D}^{b}\left(\operatorname{coh}\left(Z_{m-1}\right)\right), \ldots, \mathbf{D}^{b}\left(\operatorname{coh}\left(Z_{m-1}\right)\right)}_{n_{m-1} \text { times }},
\end{aligned}
$$




$$
\underbrace{\mathbf{D}^{b}\left(\operatorname{coh}\left(\left(Y_{m}\right)_{\text {red }}\right)\right), \ldots, \mathbf{D}^{b}\left(\operatorname{coh}\left(\left(Y_{m}\right)_{\text {red }}\right)\right)}_{n_{m} \text { times }},\rangle .
$$

Here, $n_{i}$ for $0 \leq i \leq m-1$ denotes the power of the ideal $I_{Z_{i}}$ which gives $I_{S_{i}}$, the ideal of the nonrational locus at step $i$, and $n_{m}$ stands for the nilpotence degree of the nilradical of the scheme $Y_{m}$.

\subsection{Properties of the resolution}

In this section, we discuss some properties of the categorical resolution constructed above.

For each open subset $U \subset Y$ we consider the DG-resolution of $U$ obtained by a base change from the diagram (59). To be more precise, we define $U_{0}=U$ and $U_{k+1}$ to be the blowup of $U_{k}$ with center $Z_{k}^{U}:=U_{k} \cap Z_{k}$. Moreover, for each step we choose as a nonrational locus for this blowup the subscheme $S_{k}^{U}:=U_{k} \cap S_{k}$. We denote by $\mathscr{D}_{U}$ the obtained categorical DG-resolution of $\mathscr{D}(U)$.

Proposition 6.13. The association $U \mapsto \mathscr{D}_{U}$ defines a presheaf of DG-categories on $Y$.

Proof. The proof is evident.

Remark 6.14. One can check that $\mathscr{D}_{U}$ is quasiequivalent to the Drinfeld quotient of $\mathscr{D}$ by the subcategory generated by all objects in the components $\mathscr{D}\left(Z_{k}\right)$ of $\mathscr{D}$ which are cohomologically supported on $Z_{k} \backslash Z_{k}^{U}$ as well as by all objects in $\mathscr{D}\left(Y_{m}\right)$ which are cohomologically supported on $Y_{m} \backslash U_{m}$.

Note that, for $U$ sufficiently small (contained in the complement of the union of images of all $Z_{k}$ in $Y$ ), we have $\mathscr{D}_{U} \cong \mathscr{D}(U)$. Thus, the constructed resolution is "birational".

Proposition 6.15. Let $g: Y \rightarrow Y$ be an automorphism that preserves the resolution (59). Then there is a autoequivalence $g^{*}: \mathscr{D} \rightarrow \mathscr{D}$ which preserves the semiorthogonal decompositions $(61)$ of $\mathbf{D}(\mathscr{D})$ and $\mathbf{D}(\mathscr{D})^{\mathrm{c}}$ and on each component of these decompositions is compatible with the pullback functor induced by the restriction of $g$ onto the corresponding scheme $Z_{i}$ or $Y_{m}$. Moreover, the quasiautoequivalence $g^{*}$ extends to a quasiautoequivalence of the presheaf $\mathscr{D}_{U}$. 
Proof. The proof is straightforward. Here it is important to use the functorial version of the the resolution of singularities, see [5, Theorem 1.1]

\section{Acknowledgements}

The authors are grateful to the Indiana University in Bloomington where a significant part of the work has been done. We are very grateful to Dima Kaledin, Dima Orlov, and Olaf Schnürer for valuable discussions and Osamu Iyama for insightful comments. We also thank Bernhard Keller and Bertrand Toën for answering some questions. We are especially grateful to Sasha Efimov whose suggestions allowed us to simplify considerably the section on $\mathscr{A}$-modules.

\section{Funding}

A.K. was partially supported by RFFI grant NSh-2998.2014.1, the grant of the Simons foundation, and by AG Laboratory SU-HSE, RF government grant, ag.11.G34.31.0023.

\section{Appendix 1. More on Gluings}

In this Appendix, we show that the operation of gluing described in Section 4 is compatible with tensor products of DG-categories, and provide a description of quasifunctors to and from the gluing. We use freely the notation from Section 4.

\section{A.1 The gluing and tensor products}

First, we observe that the opposite DG-category of the gluing is itself obtained by a gluing. For this, note that

$$
\left(\mathscr{D}_{2}^{\mathrm{op}} \otimes \mathscr{D}_{1}\right)-\mathrm{dgm}=\left(\left(\mathscr{D}_{1}^{\mathrm{op}}\right)^{\mathrm{op}} \otimes\left(\mathscr{D}_{2}\right)^{\mathrm{op}}\right)-\mathrm{dgm},
$$

thus any bimodule $\varphi \in\left(\mathscr{D}_{2}^{\mathrm{op}} \otimes \mathscr{D}_{1}\right)$-dgm can be also used for the gluing of $\mathscr{D}_{2}^{\text {op }}$ with $\mathscr{D}_{1}^{\text {op }}$.

Lemma A.1. One has a DG-equivalence $\left(\mathscr{D}_{1} \times_{\varphi} \mathscr{D}_{2}\right)^{\mathrm{op}} \cong \mathscr{D}_{2}^{\mathrm{op}} \times_{\varphi} \mathscr{D}_{1}^{\mathrm{op}}$.

Proof. The objects and the Hom-complexes are the same by definition.

Let $\mathcal{C}$ be a small DG-category. Consider the bimodule

$$
\bar{\varphi}:=\mathcal{C} \otimes_{\mathbb{k}} \varphi \in\left(\mathcal{C}^{\mathrm{op}} \otimes \mathcal{C} \otimes \mathscr{D}_{2}^{\mathrm{op}} \otimes \mathscr{D}_{1}\right)-\mathrm{dgm}=\left(\left(\mathcal{C} \otimes \mathscr{D}_{2}\right)^{\mathrm{op}} \otimes\left(\mathcal{C} \otimes \mathscr{D}_{1}\right)\right)-\mathrm{dgm}
$$

We can use it to form the gluing $\left(\mathcal{C} \otimes \mathscr{D}_{1}\right) \times_{\bar{\varphi}}\left(\mathcal{C} \otimes \mathscr{D}_{2}\right)$. 
Proposition A.2. There is an equivalence of categories

$$
\mathbf{D}\left(\mathcal{C} \otimes\left(\mathscr{D}_{1} \times_{\varphi} \mathscr{D}_{2}\right)\right) \cong \mathbf{D}\left(\left(\mathcal{C} \otimes \mathscr{D}_{1}\right) \times_{\bar{\varphi}}\left(\mathcal{C} \otimes \mathscr{D}_{2}\right)\right) .
$$

Proof. Consider a DG-functor $\Delta: \mathcal{C} \otimes\left(\mathscr{D}_{1} \times_{\varphi} \mathscr{D}_{2}\right) \rightarrow\left(\mathcal{C} \otimes \mathscr{D}_{1}\right) \times_{\bar{\varphi}}\left(\mathcal{C} \otimes \mathscr{D}_{2}\right)$ defined by

$$
C \otimes\left(M_{1}, M_{2}, \mu\right) \mapsto\left(C \otimes M_{1}, C \otimes M_{2}, 1_{C} \otimes \mu\right)
$$

Note that

$$
\begin{aligned}
& \operatorname{Hom}_{\left(\mathcal{C} \otimes \mathscr{D}_{1}\right) \times_{\bar{\varphi}}\left(\mathcal{C} \otimes \mathscr{D}_{2}\right)}\left(\left(C \otimes M_{1}, C \otimes M_{2}, 1_{C} \otimes \mu\right),\left(D \otimes N_{1}, D \otimes N_{2}, 1_{D} \otimes v\right)\right) \\
& \quad=\operatorname{Hom}_{\mathcal{C} \otimes \mathscr{D}_{1}}\left(C \otimes M_{1}, D \otimes N_{1}\right) \oplus \operatorname{Hom}_{\mathcal{C} \otimes \mathscr{D}_{2}}\left(C \otimes M_{2}, D \otimes N_{2}\right) \oplus \bar{\varphi}\left(D \otimes N_{2}, C \otimes M_{1}\right) \\
& =\operatorname{Hom}_{\mathcal{C}}(C, D) \otimes\left(\operatorname{Hom}_{\mathscr{D}_{1}}\left(M_{1}, N_{1}\right) \oplus \operatorname{Hom}_{\mathscr{D}_{2}}\left(M_{2}, N_{2}\right) \oplus \varphi\left(N_{2}, M_{1}\right)\right) \\
& =\operatorname{Hom}_{\mathcal{C} \otimes\left(\mathscr{D}_{1} \times_{\varphi} \mathscr{D}_{2}\right)}\left(C \otimes\left(M_{1}, M_{2}, \mu\right), D \otimes\left(N_{1}, N_{2}, v\right)\right),
\end{aligned}
$$

which means that the functor $\Delta$ is fully faithful.

By Proposition 3.9, the DG-functor $\Delta$ extends to a fully faithful triangulated functor $\operatorname{LInd}_{\Delta}: \mathbf{D}\left(\mathcal{C} \otimes\left(\mathscr{D}_{1} \times_{\varphi} \mathscr{D}_{2}\right)\right) \rightarrow \mathbf{D}\left(\left(\mathcal{C} \otimes \mathscr{D}_{1}\right) \times_{\bar{\varphi}}\left(\mathcal{C} \otimes \mathscr{D}_{2}\right)\right)$ which has a right adjoint. Thus, it remains to check that the orthogonal in $\mathbf{D}\left(\left(\mathcal{C} \otimes \mathscr{D}_{1}\right) \times_{\bar{\varphi}}\left(\mathcal{C} \otimes \mathscr{D}_{2}\right)\right)$ to the image of LInd $_{\Delta}$ is zero. For this, it suffices to check that any representable $\left(\left(\mathcal{C} \otimes \mathscr{D}_{1}\right) \times_{\bar{\varphi}}\left(\mathcal{C} \otimes \mathscr{D}_{2}\right)\right)$ module is contained in the triangulated category generated by the image of LInd $\mathrm{d}_{\Delta}$.

To check this, take any object $\left(C_{1} \otimes M_{1}, C_{2} \otimes M_{2}, \bar{\mu}\right) \in\left(\mathcal{C} \otimes \mathscr{D}_{1}\right) \times_{\bar{\varphi}}\left(\mathcal{C} \otimes \mathscr{D}_{2}\right)$. The canonical triangle (27) then shows that

$$
\left(C_{1} \otimes M_{1}, C_{2} \otimes M_{2}, \bar{\mu}\right) \cong \operatorname{Cone}\left(\left(C_{1} \otimes M_{1}, 0,0\right)[-1] \stackrel{\bar{\mu}}{\longrightarrow}\left(0, C_{2} \otimes M_{2}, 0\right)\right) .
$$

Since both $\left(C_{1} \otimes M_{1}, 0,0\right)=\Delta\left(C_{1} \otimes\left(M_{1}, 0,0\right)\right)$ and $\left(0, C_{2} \otimes M_{2}, 0\right)=\Delta\left(C_{2} \otimes\left(0, M_{2}, 0\right)\right)$ are in the image of the functor $\operatorname{LInd}_{\Delta}$, the result follows.

Remark A.3. In fact, the above argument shows also that the pretriangulated envelope of the DG-category $\mathcal{C} \otimes\left(\mathscr{D}_{1} \times_{\varphi} \mathscr{D}_{2}\right)$ is quasiequivalent to the pretriangulated envelope of $\left(\mathcal{C} \otimes \mathscr{D}_{1}\right) \times_{\bar{\varphi}}\left(\mathcal{C} \otimes \mathscr{D}_{2}\right)$.

Corollary A.4. We have a semiorthogonal decomposition

$$
\mathbf{D}\left(\mathcal{C} \otimes\left(\mathscr{D}_{1} \times_{\varphi} \mathscr{D}_{2}\right)\right)=\left\langle\mathbf{D}\left(\mathcal{C} \otimes \mathscr{D}_{1}\right), \mathbf{D}\left(\mathcal{C} \otimes \mathscr{D}_{2}\right)\right\rangle
$$

with the gluing functor equal to $-\stackrel{\mathbb{L}}{\otimes} \mathscr{D}_{2} \varphi: \mathbf{D}\left(\mathcal{C} \otimes \mathscr{D}_{2}\right) \rightarrow \mathbf{D}\left(\mathcal{C} \otimes \mathscr{D}_{1}\right)$. 
Proof. The semiorthogonal decomposition follows from the combination of Proposition A.2 and Proposition 4.6. By Proposition 4.6, the gluing functor is isomorphic to

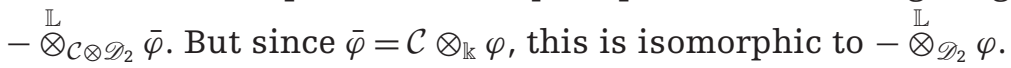

Combining this Corollary with Proposition 4.10, one easily deduces the following corollary.

Corollary A.5. Assume that $\mathscr{D}$ is a small pretriangulated DG-category with a semiorthogonal decomposition $[\mathscr{D}]=\left\langle\mathscr{T}_{1}, \mathscr{T}_{2}\right\rangle$. Then, for any small DG-category $\mathcal{C}$ there are semiorthogonal decompositions

$$
\mathbf{D}(\mathcal{C} \otimes \mathscr{D})=\left\langle\mathbf{D}\left(\mathcal{C} \otimes \mathscr{D}_{1}\right), \mathbf{D}\left(\mathcal{C} \otimes \mathscr{D}_{2}\right)\right\rangle, \quad \operatorname{Perf}(\mathcal{C} \otimes \mathscr{D})=\left\langle\operatorname{Perf}\left(\mathcal{C} \otimes \mathscr{D}_{1}\right), \operatorname{Perf}\left(\mathcal{C} \otimes \mathscr{D}_{2}\right)\right\rangle,
$$

where $\mathscr{D}_{1}$ and $\mathscr{D}_{2}$ are, respectively, the enhancements of $\mathscr{T}_{1}$ and $\mathscr{T}_{2}$ induced by $\mathscr{D}$, and Perf stands for the homotopy category of perfect DG-modules.

\section{A.2 Quasifunctors to and from the gluing}

One can describe the category of quasifunctors to and from the gluing.

Definition A.6. A DG-bimodule $\varphi \in\left(\mathscr{D}_{1}^{\mathrm{op}} \otimes \mathscr{D}_{2}\right)$-dgm is right quasirepresentable (also called a quasifunctor) if, for any $X_{1} \in \mathscr{D}_{1}$, the right $\mathscr{D}_{2}$-module $\varphi\left(X_{1},-\right)$ is quasiisomorphic to a representable DG-module.

\section{Proposition A.7.}

(i) To give a quasifunctor $\alpha: \mathcal{C} \rightarrow \mathscr{D}_{1} \times_{\varphi} \mathscr{D}_{2}$ is equivalent to giving a quasifunctor $\alpha_{1}: \mathcal{C} \rightarrow \mathscr{D}_{1}$, a quasifunctor $\alpha_{2}: \mathcal{C} \rightarrow \mathscr{D}_{2}$, and a morphism from $\alpha_{1}$ to $\varphi \circ \alpha_{2}$ in the derived category $\mathbf{D}\left(\mathcal{C}^{\mathrm{op}} \otimes \mathscr{D}_{1}\right)$.

(ii) If $\mathcal{C}$ is pretriangulated and $\varphi$ is a quasifunctor, then to give a quasifunctor $\beta: \mathscr{D}_{1} \times_{\varphi} \mathscr{D}_{2} \rightarrow \mathcal{C}$ is equivalent to giving a quasifunctor $\beta_{1}: \mathscr{D}_{1} \rightarrow \mathcal{C}$, a quasifunctor $\beta_{2}: \mathscr{D}_{2} \rightarrow \mathcal{C}$, and a morphism from $\beta_{2}$ to $\beta_{1} \circ \varphi$ in the derived category $\mathbf{D}\left(\mathscr{D}_{2}^{\mathrm{op}} \otimes \mathcal{C}\right)$.

Proof. (i) We apply Proposition A.2 with $\mathcal{C}^{\text {op }}$ instead of $\mathcal{C}$. By Lemma 2.5, to give an object $\alpha \in \mathbf{D}\left(\mathcal{C}^{\mathrm{op}} \otimes \mathscr{D}\right)$ is equivalent to giving its components $\alpha_{1}=I_{1}^{*} \alpha \in \mathbf{D}\left(\mathcal{C}^{\mathrm{op}} \otimes \mathscr{D}_{1}\right)$ and $\alpha_{2}=I_{2}^{!} \alpha \in \mathbf{D}\left(\mathcal{C}^{\text {op }} \otimes \mathscr{D}_{2}\right)$ and a morphism $\alpha_{1} \rightarrow \alpha_{2} \stackrel{\mathbb{L}}{\otimes} \mathscr{D}_{2} \varphi=\varphi \circ \alpha_{2}$. Thus, we only have to 
check that $\alpha$ is a quasifunctor if and only if both its components $\alpha_{1} \in \mathbf{D}\left(\mathcal{C}^{\text {op }} \otimes \mathscr{D}_{1}\right)$ and $\alpha_{2} \in \mathbf{D}\left(\mathcal{C}^{\mathrm{op}} \otimes \mathscr{D}_{2}\right)$ are. Substituting $C$ into distinguished triangle

$$
I_{2} \alpha_{2} \rightarrow \alpha \rightarrow I_{1} \alpha_{1}
$$

we obtain a distinguished triangle

$$
\left(I_{2} \alpha_{2}\right)(C,-) \rightarrow \alpha(C,-) \rightarrow\left(I_{1} \alpha_{1}\right)(C,-)
$$

Note also that

$$
\left(I_{k} \alpha_{k}\right)(C,-)=\left(\alpha_{k} \stackrel{\mathbb{L}}{\otimes} \mathscr{D}_{k} i_{k} \mathscr{D}\right)(C,-)=\alpha_{k}(C,-) \stackrel{\mathbb{L}}{\otimes} \mathscr{D}_{k} \mathscr{D}\left(i_{k}(-),-\right) \cong I_{k}\left(\alpha_{k}(C,-)\right) .
$$

Thus, $\alpha_{k}(C,-) \in \mathbf{D}\left(\mathscr{D}_{k}\right) \subset \mathbf{D}(\mathscr{D})$ are just the components of $\alpha(C,-)$ with respect to the semiorthogonal decomposition $\mathbf{D}(\mathscr{D})=\left\langle\mathbf{D}\left(\mathscr{D}_{1}\right), \mathbf{D}\left(\mathscr{D}_{2}\right)\right\rangle$.

Now assume that $\alpha$ is a quasifunctor, so $\alpha(C,-) \cong Y^{\left(M_{1}, M_{2}, \mu\right)}$. By the above argument $\alpha_{k}(C,-) \in \mathbf{D}\left(\mathscr{D}_{k}\right)$ are just the components of $\mathrm{Y}^{\left(M_{1}, M_{2}, \mu\right)}$ in $\mathbf{D}\left(\mathscr{D}_{k}\right)$. The latter are evidently given by $\mathrm{Y}^{M_{k}}$. Hence $\alpha_{1}$ and $\alpha_{2}$ are quasifunctors.

Conversely, if both $\alpha_{1}$ and $\alpha_{2}$ are quasifunctors and $I_{k}\left(\alpha_{k}(C,-)\right) \cong I_{k}\left(\mathrm{Y}^{M_{k}}\right) \cong Y^{i_{k}\left(M_{k}\right)}$, we deduce that $\alpha(C,-)$ fits into the triangle

$$
\mathrm{Y}^{i_{2}\left(M_{2}\right)} \rightarrow \alpha(C,-) \rightarrow \mathrm{Y}^{i_{1}\left(M_{1}\right)}
$$

in $\mathbf{D}(\mathscr{D})=\left\langle\mathbf{D}\left(\mathscr{D}_{1}\right), \mathbf{D}\left(\mathscr{D}_{2}\right)\right\rangle$. Thus, $\alpha(C,-)$ is quasiisomorphic to the cone of a morphism from $Y^{M_{1}}[-1]$ to $Y^{M_{2}}$. The space of such morphisms is nothing but the zero cohomology of the complex $\varphi\left(M_{2}, M_{1}\right)$, and if we take the morphism corresponding to some closed element $\mu \in \varphi\left(M_{2}, M_{1}\right)$ of degree zero, we will obtain precisely $\mathrm{Y}^{\left(M_{1}, M_{2}, \mu\right)}$. Thus, we have $\alpha(C,-) \cong Y^{\left(M_{1}, M_{2}, \mu\right)}$ for appropriate $\mu$, hence $\alpha$ is a quasifunctor.

(ii) Analogously, we apply Proposition A.2 with $\mathscr{D}^{\mathrm{op}}=\mathscr{D}_{2}^{\mathrm{op}} \times_{\varphi} \mathscr{D}_{1}^{\mathrm{op}}$ instead of $\mathscr{D}$. Note that the order of DG-categories $\mathscr{D}_{1}$ and $\mathscr{D}_{2}$ in the gluing is interchanged. By this reason the embedding and the projection functors of the associated semiorthogonal decompositions indexed by 1 (such as $i_{1}, I_{1}, I_{1}^{*}$ and so on) are related to the category $\mathscr{D}_{2}$ and those indexed by 2 to the category $\mathscr{D}_{1}$.

According to Lemma 2.5, to give an object $\beta \in \mathbf{D}\left(\mathscr{D}^{\mathrm{op}} \otimes \mathcal{C}\right)$ is equivalent to giving its components $\beta_{2}=I_{1}^{*} \beta \in \mathbf{D}\left(\mathscr{D}_{2}^{\text {op }} \otimes \mathcal{C}\right)$ and $\beta_{1}=I_{2}^{!} \beta \in \mathbf{D}\left(\mathscr{D}_{1}^{\text {op }} \otimes \mathcal{C}\right)$ and a morphism $\beta_{2} \rightarrow \varphi \stackrel{\mathbb{L}}{\otimes} \mathscr{D}_{1} \beta_{1}=\beta_{1} \circ \varphi$. Thus, we only have to check that $\beta$ is a quasifunctor if and only if both its components $\beta_{2} \in \mathbf{D}\left(\mathscr{D}_{2}^{\text {op }} \otimes \mathcal{C}\right)$ and $\beta_{1} \in \mathbf{D}\left(\mathscr{D}_{1}^{\text {op }} \otimes \mathcal{C}\right)$ are. 
So, assume that $\beta$ is a quasifunctor. Then $\beta_{1}\left(M_{1},-\right)=\left(I_{2}^{!} \beta\right)\left(M_{1},-\right)=$ $\beta\left(\left(M_{1}, 0,0\right),-\right)$ is quasirepresentable, so $\beta_{1}$ is a quasifunctor. The case of $\beta_{2}$ is slightly more complicated. We have to check that $\beta_{2}\left(M_{2},-\right)=\left(I_{1}^{*} \beta\right)\left(M_{2},-\right)$ is quasirepresentable. Since $\mathcal{C}$ is pretriangulated, using triangle (26) we see that it suffices to check quasirepresentability of DG-modules $\beta_{i_{1}}\left(M_{2},-\right)=\beta\left(\left(0, M_{2}, 0\right),-\right)$ and of $\left(\varphi \stackrel{\mathbb{L}}{\otimes} \mathscr{D}_{1} \beta_{i_{2}}\right)\left(M_{2},-\right)=\varphi\left(M_{2},-\right) \stackrel{\mathbb{L}}{\otimes} \mathscr{D}_{1} \beta_{i_{2}}$. The first holds since $\beta$ is a quasifunctor. For the second, note that since $\varphi$ is a quasifunctor, we know that $\varphi\left(M_{2},-\right) \cong Y^{N_{1}}$ for some $N_{1} \in \mathscr{D}_{1}$. Therefore,

$$
\varphi\left(M_{2},-\right) \stackrel{\mathbb{L}}{\otimes} \mathscr{D}_{1} \beta_{i_{2}} \cong \mathrm{Y}^{N_{1}} \otimes_{\mathscr{D}_{1}} \beta_{i_{2}}=\left(\beta_{i_{2}}\right)\left(N_{1},-\right)=\beta\left(\left(N_{1}, 0,0\right),-\right) .
$$

This latter DG-module is quasirepresentable since $\beta$ is a quasifunctor. Combining all this, we conclude that $\beta_{2}$ is also a quasifunctor.

Conversely, assume that $\beta_{1}$ and $\beta_{2}$ are quasifunctors. Then

$$
\left(I_{1} \beta_{2}\right)\left(\left(M_{1}, M_{2}, \mu\right),-\right)=\left(\mathscr{D}_{i_{1}} \stackrel{\mathbb{L}}{\otimes} \mathscr{D}_{2} \beta_{2}\right)\left(\left(M_{1}, M_{2}, \mu\right),-\right) .
$$

Note that

$$
\left(\mathscr{D}_{i_{1}}\right)\left(\left(M_{1}, M_{2}, \mu\right), N_{2}\right)=\operatorname{Hom}_{\mathscr{D}}\left(\left(0, N_{2}, 0\right),\left(M_{1}, M_{2}, \mu\right)\right)=\operatorname{Hom}_{\mathscr{D}_{2}}\left(N_{2}, M_{2}\right)=\mathrm{Y}^{M_{2}}\left(N_{2}\right)
$$

Thus, the above tensor product equals $\mathrm{Y}^{M_{2}} \stackrel{\mathbb{L}}{\otimes} \mathscr{D}_{2} \beta_{2}=\beta_{2}\left(M_{2},-\right)$. Since $\beta_{2}$ is a quasifunctor, this $\mathcal{C}$-DG-module is quasirepresentable.

Analogously,

$$
\left(I_{2} \beta_{1}\right)\left(\left(M_{1}, M_{2}, \mu\right),-\right)=\left(\mathscr{D}_{i_{2}} \stackrel{\mathbb{L}}{\otimes} \mathscr{D}_{1} \beta_{1}\right)\left(\left(M_{1}, M_{2}, \mu\right),-\right)
$$

and we have

$$
\left(\mathscr{D}_{i_{2}}\right)\left(\left(M_{1}, M_{2}, \mu\right), N_{1}\right)=\operatorname{Hom}_{\mathscr{D}}\left(\left(N_{1}, 0,0\right),\left(M_{1}, M_{2}, \mu\right)\right)=\operatorname{Hom}_{\mathscr{D}_{1}}\left(N_{1}, M_{1}\right) \oplus \varphi\left(M_{2}, N_{1}\right)[-1]
$$

with the differential coinciding with that of Cone $\left(Y^{M_{1}} \stackrel{\mu}{\longrightarrow} \varphi\left(M_{2},-\right)\right)[-1]$ evaluated on $N_{1}$. Therefore, the above tensor product is quasiisomorphic to

$$
\text { Cone }\left(\mathrm{Y}^{M_{1}} \stackrel{\mathbb{L}}{\otimes} \mathscr{D}_{1} \beta_{1} \stackrel{\mu}{\longrightarrow} \varphi\left(M_{2},-\right) \stackrel{\mathbb{L}}{\otimes} \mathscr{D}_{1} \beta_{1}\right)[-1]
$$

The first term here is quasiisomorphic to $\beta_{1}\left(M_{1},-\right)$, and so is quasirepresentable. On the other hand, since $\varphi$ is a quasifunctor, $\varphi\left(M_{2},-\right) \cong Y^{N_{1}}$ for some $N_{1} \in \mathscr{D}_{1}$, hence the second 
term is quasiisomorphic to $Y^{N_{1}} \otimes \mathscr{D}_{1} \beta_{1}=\beta_{1}\left(N_{1},-\right)$, so it is also quasirepresentable. Since $\mathcal{C}$ is pretriangulated, the above cone is also quasirepresentable.

Thus, we have checked that both $\left(I_{1} \beta_{2}\right)\left(\left(M_{1}, M_{2}, \mu\right),-\right)$ and $\left(I_{2} \beta_{1}\right)\left(\left(M_{1}, M_{2}, \mu\right),-\right)$ are quasirepresentable $\mathcal{C}$-modules, hence so is $\beta\left(\left(M_{1}, M_{2}, \mu\right),-\right)$ as $\mathcal{C}$ is pretriangulated.

\section{Appendix 2. More on Auslander Algebras}

\section{A.3 Quiver sheaves}

In this section, we give an alternative description of the category of $\mathscr{A}$-modules and translate most of the constructions of Section 5 to this language.

Definition A.8. A quasicoherent quiver sheaf on $(S, \mathfrak{r}, n)$ is

(1) a collection $M_{1}, \ldots, M_{n}$ of quasicoherent sheaves on $S$,

(2) a collection of morphisms $\alpha: M_{i} \rightarrow M_{i+1}$,

(3) a collection of morphisms $\beta: M_{i} \otimes_{\mathcal{O}_{S}} \mathfrak{r} \rightarrow M_{i-1}$ ，

such that

(1) the diagram

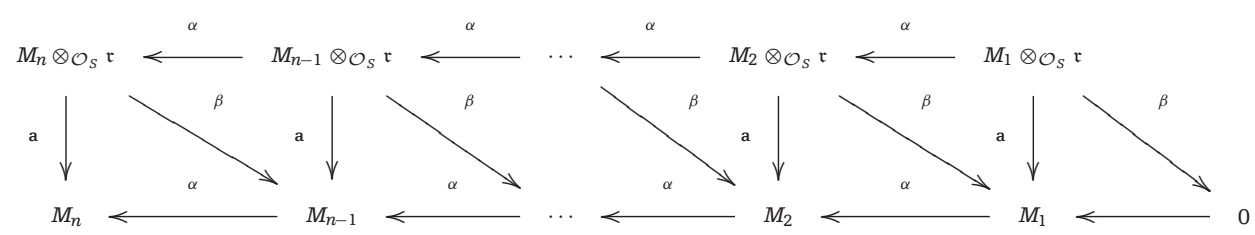

(where a : $M_{i} \otimes_{\mathcal{O}_{S}} \mathfrak{r} \rightarrow M_{i}$ is the action of $\mathfrak{r}$ on $M_{i}$ ) is commutative;

(2) for all $i$ and $k$ there is a map $\beta_{k}: M_{i} \otimes_{\mathcal{O}_{S}} \mathfrak{r}^{k} \rightarrow M_{i-k}$ such that the diagram

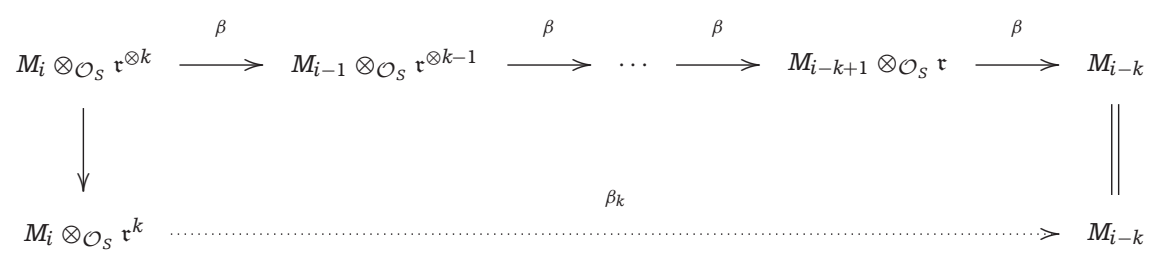


commutes, where the vertical arrow is the map $M_{i} \otimes_{\mathcal{O}_{S}} \mathfrak{r}^{\otimes k} \rightarrow M_{i} \otimes_{\mathcal{O}_{S}} \mathfrak{r}^{k}$ given by the multiplication in $\mathfrak{r}$.

A morphism of quiver sheaves from $\bar{M}$ to $\bar{N}$ is a collection of morphisms of quasicoherent sheaves $f_{i}: M_{i} \rightarrow N_{i}$ commuting with $\alpha$ and $\beta$.

Lemma A.9. The category of quiver sheaves is equivalent to the category Qcoh( $\mathscr{A})$ of quasicoherent $\mathscr{A}$-modules.

Proof. Let $\bar{M}=\left(M_{n}, \ldots, M_{1}\right)$ be a quiver sheaf. Then, we consider a quasicoherent sheaf $M:=M_{n} \oplus M_{n-1} \oplus \cdots \oplus M_{1}$ on $S$ and define a structure of a right $\mathscr{A}$-module on it as follows. For each $i, j$ we consider the map

$$
M_{n+1-i} \otimes \mathscr{A}_{i j}=M_{n+1-i} \otimes\left(\mathfrak{r}^{\max (j-i, 0)} / \mathfrak{r}^{n+1-i}\right) \rightarrow M_{n+1-j}
$$

which is given by the map $\beta_{j-i}$ if $j \geq i$ and by the map $\alpha^{i-j}$ if $j<i$. Note that by part (2) of Definition A.8 the ideal $\mathfrak{r}^{n+1-i}$ acts trivially on $M_{n+1-i}$, so the above map is well defined.

Conversely, assume that $M$ is a quasicoherent $\mathscr{A}$-module. Using idempotents $\epsilon_{i}$ we define $M_{i}=M \epsilon_{n+1-i}$. This gives a decomposition $M=M_{n} \oplus M_{n-1} \oplus \cdots \oplus M_{1}$. Now, we equip $\bar{M}=\left(M_{n}, M_{n-1}, \ldots, M_{1}\right)$ with a structure of a quiver sheaf by defining the map $\alpha: M_{n-i} \rightarrow M_{n+1-i}$ as a map given by the element $\alpha_{i}=1 \in \mathcal{O}_{S} / \mathfrak{r}^{n-i}=\mathscr{A}_{i+1, i}$, and the map $\beta: M_{n+1-i} \otimes \mathfrak{r} \rightarrow M_{n-i}$ as a map induced by the action of $\mathscr{A}_{i, i+1}=\mathfrak{r} / \mathfrak{r}^{n+1-i}$.

It is a straightforward exercise left to the reader to check that these are mutually inverse equivalences.

It is easy to write down the functors of the semiorthogonal decomposition in terms of quiver sheaves. An easy verification shows that

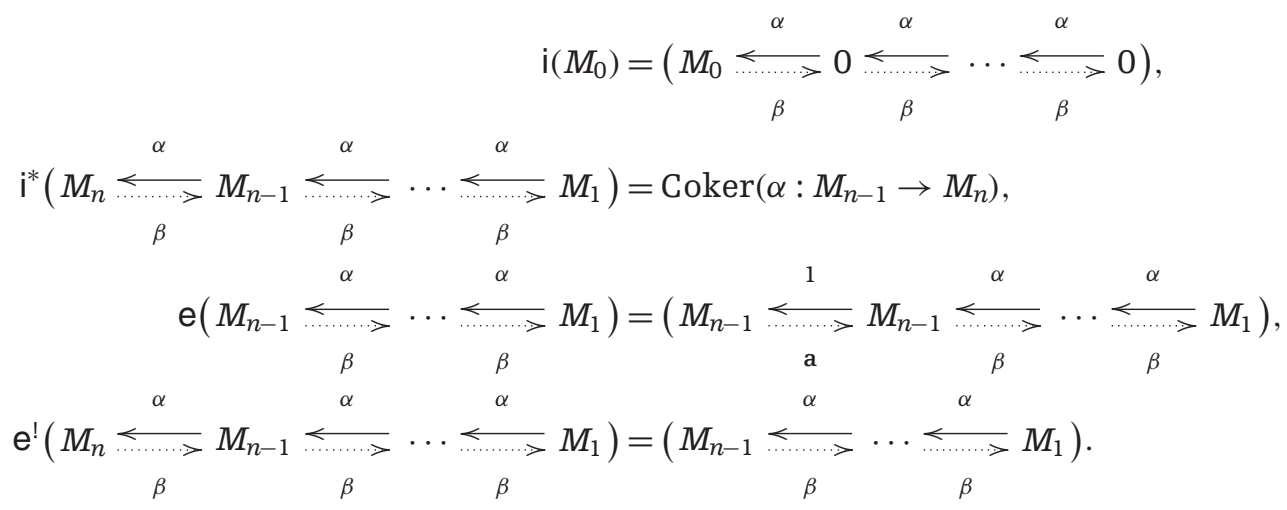


It is also easy to write down the resolution functors

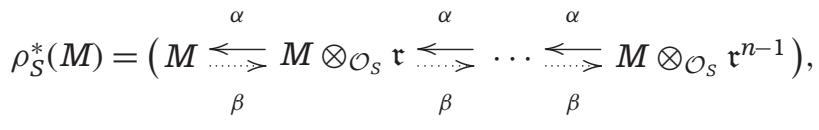

$$
\begin{aligned}
& \rho_{S *}\left(M_{n} \stackrel{\alpha}{\longleftarrow} M_{n-1} \stackrel{\alpha}{\longleftarrow} \ldots \stackrel{\alpha}{\longleftarrow} \stackrel{\alpha}{>}_{>} M_{1}\right)=M_{n} \text {, } \\
& \beta \quad \beta \quad \beta
\end{aligned}
$$

where, in the first line morphisms $\alpha$ are induced by the injections $\mathfrak{r}^{i} \rightarrow \mathfrak{r}^{i-1}$, and morphisms $\beta$ are induced by the multiplication $\mathfrak{r}^{i} \otimes_{\mathcal{O}_{S}} \mathfrak{r} \rightarrow \mathfrak{r}^{i+1}$.

\section{A.4 Bounded coherent sheaves on a nonreduced scheme}

In this section, we show that the functor $\rho_{S *}: \mathbf{D}^{b}(\operatorname{coh}(\mathscr{A})) \rightarrow \mathbf{D}^{b}(\operatorname{coh}(S))$ is a localization of triangulated categories. First note that the statement is evident on the level of big categories.

Lemma A.10. The category $\mathbf{D}(S)$ is a localization of the category $\mathbf{D}(\mathscr{A})$.

Proof. Since the functor $L \rho_{S}^{*}: \mathbf{D}(S) \rightarrow \mathbf{D}(\mathscr{A})$ is fully faithful and has a right adjoint, we have a semiorthogonal decomposition

$$
\mathbf{D}\left(\mathscr{A}_{S}\right)=\left\langle\operatorname{Ker} \rho_{S *}, \mathbf{D}(S)\right\rangle
$$

In particular, $\mathbf{D}(S)$ is equivalent to the localization of $\mathbf{D}(\mathscr{A})$ by $\operatorname{Ker} \rho_{S *}$.

The only problem with extending this result to bounded coherent categories is that the functor $L \rho_{S}^{*}$ does not preserve boundedness. By definition (47), it is given by a tensor product with a nonperfect $\mathcal{O}_{S}$-module, hence $L \rho_{S}^{*}(M)$ is unbounded from below. So, for example, if we want to show that, for any $M \in \mathbf{D}^{b}(\operatorname{coh}(S))$, there is $\mathcal{M} \in \mathbf{D}^{b}(\operatorname{coh}(\mathscr{A}))$ such that $M \cong \rho_{S *}(\mathcal{M})$, we cannot just take $\mathcal{M}=L \rho_{S}^{*}(M)$. On the other hand, we can take $\mathcal{M}$ to be a suitable truncation of $L \rho_{S}^{*}(M)$ with respect to the standard $t$-structure.

Lemma A.11. The functor $\rho_{S_{*}}: \mathbf{D}^{b}(\operatorname{coh}(\mathscr{A})) \rightarrow \mathbf{D}^{b}(\operatorname{coh}(S))$ is t-exact with respect to the standard $t$-structures. In other words,

$$
\rho_{S *} \circ \tau^{\leq k}=\tau^{\leq k} \circ \rho_{S *}, \quad \rho_{S *} \circ \tau^{\geq k}=\tau^{\geq k} \circ \rho_{S *},
$$


where $\tau^{\leq k}$ and $\tau^{\geq k}$ are the truncation functors of the standard t-structures on $\mathbf{D}^{b}(\operatorname{coh}(\mathscr{A}))$ and $\mathbf{D}^{b}(\operatorname{coh}(S))$.

Proof. Recall that $\rho_{S *}(M)=M \epsilon_{1}$, the direct summand corresponding to the idempotent $\epsilon_{1}$ of $\mathscr{A}$. So it is evidently $t$-exact.

We start with the following preparatory result.

Proposition A.12. Any morphism in $\mathbf{D}^{b}(\operatorname{coh}(S))$ can be lifted to $\mathbf{D}^{b}(\operatorname{coh}(\mathscr{A}))$. In other words, for any morphism $f: M \rightarrow N$ in $\mathbf{D}^{b}(\operatorname{coh}(S))$ there are objects $\mathcal{M}, \mathcal{N} \in \mathbf{D}^{b}(\operatorname{coh}(\mathscr{A}))$ and a morphism $\bar{f}: \mathcal{M} \rightarrow \mathcal{N}$ such that $\rho_{S *}(\mathcal{M}) \cong M, \rho_{S *}(\mathcal{N}) \cong N$ and the diagram

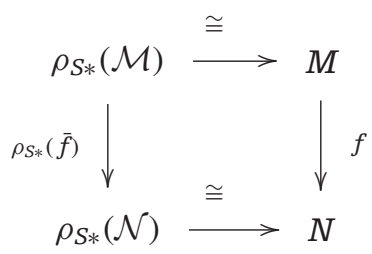

commutes.

Proof. First, let us show that, for any $M \in \mathbf{D}^{b}(\operatorname{coh}(S))$, there is an object $\mathcal{M} \in \mathbf{D}^{b}(\operatorname{coh}(\mathscr{A}))$ such that $\rho_{S_{*}} \mathcal{M} \cong M$. Indeed, by (A.1),

$$
\tau^{\geq k} M=\tau^{\geq k} \rho_{S *} L \rho_{S}^{*} M=\rho_{S *}\left(\tau^{\geq k} L \rho_{S}^{*} M\right) .
$$

Now, choose $k$ such that $\tau^{\geq k} M \cong M$ (this is possible since $M$ has bounded cohomology) and note that $\tau^{\geq k} L \rho_{S}^{*} M \in \mathbf{D}^{b}(\operatorname{coh}(\mathscr{A}))$.

The same argument applies to morphisms. Again, take $k$ such that $\tau{ }^{\geq k} M=M$ and $\tau^{\geq k} N=N$ and consider the morphism $\tau^{\geq k} L \rho_{S}^{*} M \stackrel{\tau^{\geq k} L \rho_{S}^{*} f}{\longrightarrow} \tau^{\geq k} L \rho_{S}^{*} N$. Since the truncation is functorial and comes with a morphism of functors id $\rightarrow \tau^{\geq k}$, the result follows.

Corollary A.13. The category $\mathbf{D}^{b}(\operatorname{coh}(S))$ is the quotient of $\mathbf{D}^{b}(\operatorname{coh}(\mathscr{A}))$ by $\operatorname{Ker} \rho_{S *}$.

Proof. By definition of the quotient category the functor $\rho_{S *}$ factors through a functor

$$
\mathbf{D}^{b}(\operatorname{coh}(\mathscr{A})) / \operatorname{Ker} \rho_{S *} \longrightarrow \mathbf{D}^{b}(\operatorname{coh}(S)),
$$

and we have to check that this functor is an equivalence. Note that it is essentially surjective on objects by Proposition A.12. Let us check that it is fully faithful. 
First, let us check that the functor is surjective on morphisms. Take arbitrary objects $M=\rho_{S *} \mathcal{M}, N=\rho_{S *} \mathcal{N}$ and consider a morphism $f: \rho_{S_{*}} \mathcal{M} \rightarrow \rho_{S *} \mathcal{N}$. Our goal is to construct a morphism $\mathcal{M} \rightarrow \mathcal{N}$ which goes to $f$ under $\rho_{S *}$. Note that, by Proposition A.12, there is a morphism $\bar{f}: \mathcal{M}^{\prime} \rightarrow \mathcal{N}^{\prime}$ in $\mathbf{D}^{b}(\operatorname{coh}(\mathscr{A}))$ such that diagram

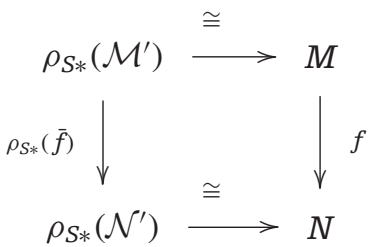

commutes. By adjunction it gives a morphism $L \rho_{S}^{*} \rho_{S *} \mathcal{M}^{\prime} \rightarrow \mathcal{M}$. On the other hand, we have an adjunction morphism $L \rho_{S}^{*} \rho_{S *} \mathcal{M}^{\prime} \rightarrow \mathcal{M}^{\prime}$. Since both $\mathcal{M}$ and $\mathcal{M}^{\prime}$ have finite number of cohomology sheaves, there exists $k$ such that both maps factor through $\tau^{\geq k} L \rho_{S}^{*} \rho_{S *} \mathcal{M}^{\prime}$. Moreover, the argument of Proposition A.12 shows that $k$ may be chosen in such a way that after application of $\rho_{S *}$ both maps will be isomorphisms. Thus, their cones are in $\operatorname{Ker} \rho_{S_{*}}$, hence both maps are isomorphisms in the quotient category. Therefore, $\mathcal{M}^{\prime}$ and $\mathcal{M}$ are isomorphic in the quotient category, hence the morphism $\bar{f}$ gives a morphism from $\mathcal{M}$ to $\mathcal{N}$ which maps into $f$ by $\rho_{S *}$. Thus, (A.2) is surjective on morphisms.

Now consider a morphism $\bar{f}: \mathcal{M} \rightarrow \mathcal{N}$ and assume that $\rho_{S *} \bar{f}: \rho_{S *} \mathcal{M} \rightarrow \rho_{S_{*}} \mathcal{N}$ is zero. By adjunction the composition $L \rho_{S}^{*} \rho_{S *} \mathcal{M} \rightarrow \mathcal{M} \stackrel{\bar{f}}{\rightarrow} \mathcal{N}$ is zero. But since both $\mathcal{M}$ and $\mathcal{N}$ are bounded, it follows that there is $k$ such that the composition $\tau^{\geq k} L \rho_{S}^{*} \rho_{S *} \mathcal{M} \rightarrow \mathcal{M} \stackrel{\bar{f}}{\rightarrow} \mathcal{N}$ is zero and $\rho_{S *}\left(\tau{ }^{\geq k} L \rho_{S}^{*} \rho_{S *} \mathcal{M}\right) \cong \rho_{S *} \mathcal{M}$. Therefore, the cone of the map $\tau^{\geq k} L \rho_{S}^{*} \rho_{S *} \mathcal{M} \rightarrow \mathcal{M}$ is in $\operatorname{Ker} \rho_{S *}$, hence the first map is an isomorphism in the quotient category, and hence $\bar{f}$ in the quotient category is zero. Thus, (A.2) is injective on morphisms and we are done.

\section{A.5 The opposite Auslander algebra}

Besides the usual Auslander algebra one can consider its opposite $\mathscr{A}^{\mathrm{op}}$. It turns out that most of the properties established for the Auslander algebra hold for the opposite as well. As an illustration we sketch a construction of the semiorthogonal decomposition analogous to that of Section 5.4.

First, we have a functor $\mathrm{i}_{\text {op }}: \mathbf{D}\left(S_{0}\right) \rightarrow \mathbf{D}\left(\mathscr{A}^{\text {op }}\right)$ defined by the same formula (36) as the functor i, as well as its (derived) left adjoint

$$
L \mathrm{i}_{\mathrm{op}}^{*}: \mathbf{D}\left(\mathscr{A}^{\mathrm{op}}\right) \rightarrow \mathbf{D}\left(S_{0}\right), \quad M \mapsto \mathcal{O}_{S_{0}} \stackrel{\mathbb{L}}{\otimes} \mathscr{A} M
$$


We also have an adjoint pair of functors

$$
\begin{aligned}
& L \mathrm{e}_{\mathrm{op}}: \mathbf{D}\left(\mathscr{A}^{\prime \mathrm{op}}\right) \rightarrow \mathbf{D}\left(\mathscr{A}^{\mathrm{op}}\right), \quad M \mapsto \mathscr{A}\left(1-\epsilon_{1}\right) \stackrel{\mathbb{L}}{\otimes} \mathscr{A}^{\prime} M, \\
& \mathrm{e}_{\mathrm{op}}: \mathbf{D}\left(\mathscr{A}^{\mathrm{op}}\right) \rightarrow \mathbf{D}\left(\mathscr{A}^{\mathrm{op}}\right), \quad N \mapsto \operatorname{Hom}_{\mathscr{A}}\left(\mathscr{A}\left(1-\epsilon_{1}\right), N\right)=\left(1-\epsilon_{1}\right) N
\end{aligned}
$$

(so the only difference is that the functor $e_{o p}$ is not exact and we have to take its derived functor). These functors still enjoy the analogs of relations (43), and tensoring (44) by arbitrary $M \in \mathbf{D}\left(\mathscr{A}^{\mathrm{op}}\right)$, we obtain an analog of triangle (46). Thus, there is a semiorthogonal decomposition

$$
\mathbf{D}\left(\mathscr{A}^{\mathrm{op}}\right)=\left\langle\mathrm{i}_{\mathrm{op}}\left(\mathbf{D}\left(S_{0}\right)\right), L \mathrm{e}_{\mathrm{op}}\left(\mathbf{D}\left(\mathscr{A}^{\prime \mathrm{op}}\right)\right)\right\rangle
$$

By iteration, we deduce an $n$-component semiorthogonal decomposition

$$
\mathbf{D}\left(\mathscr{A}^{\mathrm{op}}\right)=\left\langle\mathbf{D}\left(S_{0}\right), \ldots, \mathbf{D}\left(S_{0}\right)\right\rangle
$$

\section{A.6 Homological dimension}

Recall that the projective dimension $\operatorname{pdim}_{A}(M)$ of a right module $M$ over an algebra $A$ is the minimal length of its projective resolution. Furthermore, the global dimension gldim $A$ of $A$ is the supremum of the projective dimensions of right $A$-modules.

Proposition A.14. Assume that $S$ is a regular local scheme of dimension $d$. Then

$$
\operatorname{gldim} \mathscr{A}_{S, \mathfrak{r}, n} \leq n d+2(n-1), \quad \operatorname{gldim} \mathscr{A}_{S, \mathfrak{r}, n}^{\mathrm{op}} \leq n d+2(n-1) .
$$

Proof. We are going to prove the claim by induction on $n$. The case $n=1$ is evidentin this case $\mathscr{A}=\mathcal{O}_{S_{0}}$ is a regular local ring, so its global dimension equals its Krull dimension, which agrees with the statement of the proposition.

Now assume the claim is known for $n-1$. Note that, by (35) and (30), as a left $\mathscr{A}$-module we have $\mathscr{I} \cong \mathscr{A} \epsilon_{2} \oplus \mathscr{A}\left(1-\epsilon_{1}\right)$; in particular, it is projective, so

$$
\operatorname{pdim}_{\mathscr{A}^{\circ}} \mathscr{I}=0 \text {. }
$$

On the other hand, $\left(1-\epsilon_{1}\right) \mathscr{A}$ is projective as a right $\mathscr{A}$-module, hence the functor $\mathrm{e}(-)=$ $-{\stackrel{\mathbb{Q}}{\otimes} \mathscr{A}^{\prime}}\left(1-\epsilon_{1}\right) \mathscr{A}$ does not increase the projective dimension of a right $\mathscr{A}^{\prime}$-module. This 
means that

$$
\operatorname{pdim}_{\mathscr{A}} \mathscr{I} \leq \operatorname{gldim} \mathscr{A}^{\prime}
$$

Using (44), (A.4), and (A.5), we conclude that

$$
\operatorname{pdim}_{\mathscr{A}{ }^{\circ \mathrm{p}}} \mathcal{O}_{S_{0}} \leq 1, \quad \operatorname{pdim}_{\mathscr{A}} \mathcal{O}_{S_{0}} \leq \operatorname{gldim} \mathscr{A}^{\prime}+1
$$

Now let $M$ be a right $\mathscr{A}$-module and consider the canonical triangle

$$
\mathrm{e}\left(\mathrm{e}^{!}(M)\right) \rightarrow M \rightarrow \mathrm{i}\left(L \mathrm{i}^{*}(M)\right)
$$

Note also that we have

$$
\mathrm{i}\left(L \mathrm{i}^{*}(M)\right)=M \stackrel{\mathbb{L}}{\otimes} \mathcal{O}_{S_{0}}
$$

It follows from the first part of (A.6) that $L i^{*}(M)$ has cohomology only in degrees 0 and -1 , hence it has a free over $\mathcal{O}_{S_{0}}$ resolution of length $d+1$. On the other hand, by the second part of (A.6) i $\left(\mathcal{O}_{S_{0}}\right)$ has a projective resolution of length gldim $\mathscr{A}^{\prime}+1$. Thus, $\mathrm{i}\left(L \mathrm{i}^{*}(M)\right)$ has a projective resolution of length gldim $\mathscr{A}^{\prime}+d+2$. On the other hand, since $\mathrm{e}^{\mathrm{!}}$ is exact, $\mathrm{e}^{!}(M)$ is an $\mathscr{A}^{\prime}$-module, hence pdim $\mathscr{A}^{\prime}\left(\mathrm{e}^{!}(M)\right) \leq$ gldim $\mathscr{A}^{\prime}$, and since the functor e does not increase the projective dimension, the module $\mathrm{e}\left(\mathrm{e}^{!}(M)\right)$ has a projective resolution of length gldim $\mathscr{A}^{\prime}$. Combining these two observations, we conclude that gldim $\mathscr{A} \leq$ gldim $\mathscr{A}^{\prime}+d+2$. So, the induction hypothesis gldim $\mathscr{A}^{\prime} \leq(n-1) d+2(n-2)$ implies that we have gldim $\mathscr{A} \leq n d+2(n-1)$.

Analogously, let $M$ be a left $\mathscr{A}$-module and consider the canonical triangle

$$
L \mathrm{e}_{\mathrm{op}}\left(\mathrm{e}_{\mathrm{op}}^{!}(M)\right) \rightarrow M \rightarrow \mathrm{i}_{\mathrm{op}}\left(L \mathrm{i}_{\mathrm{op}}^{*}(M)\right)
$$

Now, by the second part of (A.6) we know that $L i_{\text {op }}^{*}(M)$ has cohomology only in degrees from 0 to $-\left(\right.$ gldim $\left.\mathscr{A}^{\text {op }}+1\right)$, hence it has a free over $\mathcal{O}_{S_{0}}$ resolution of length gldim $\mathscr{A}^{\prime \mathrm{op}}+d+1$. On the other hand, by the first part of (A.6), $\mathrm{i}_{\mathrm{op}}\left(\mathcal{O}_{S_{0}}\right)$ has a projective resolution of length 1 . Thus, $\mathrm{i}_{\mathrm{op}}\left(L \mathrm{i}_{\mathrm{op}}^{*}(M)\right)$ has a projective resolution of length gldim $\mathscr{A}^{\prime \mathrm{op}}+d+2$. On the other hand, since $\mathrm{e}_{\mathrm{op}}^{!}$is exact, $\mathrm{e}_{\mathrm{op}}^{!}(M)$ is an $\mathscr{A}^{\prime}$-module, hence $\operatorname{pdim}_{\mathscr{A}^{\prime \text { op }}}\left(\mathrm{e}^{!}(M)\right) \leq \operatorname{gldim} \mathscr{A}^{\prime \mathrm{op}}$. Since $\mathscr{A}\left(1-\epsilon_{1}\right)$ is a projective left $\mathscr{A}$-module, the functor $L \mathrm{e}_{\mathrm{op}}$ takes projective resolutions to projective resolutions of the same length, so $\mathrm{e}_{\mathrm{op}}\left(\mathrm{e}_{\mathrm{op}}^{!}(M)\right)$ has a projective resolution of length gldim $\mathscr{A}^{\prime \mathrm{op}}$. Combining these two observations, we again conclude that gldim $\mathscr{A}^{\mathrm{op}} \leq \operatorname{gldim} \mathscr{A}^{\text {op }}+d+2$. Using the induction hypothesis in the same way as above, we deduce the claim. 


\section{A.7 Generalized Auslander spaces}

In this section, we indicate how the algebra $\mathscr{A}$ can be generalized.

Let $S$ be a scheme. Choose a collection of ideals $\mathfrak{a}_{i j} \subset \mathcal{O}_{S}$, where $1 \leq i \leq n$ and $2 \leq j \leq n+1$, and the following incidence conditions are satisfied:

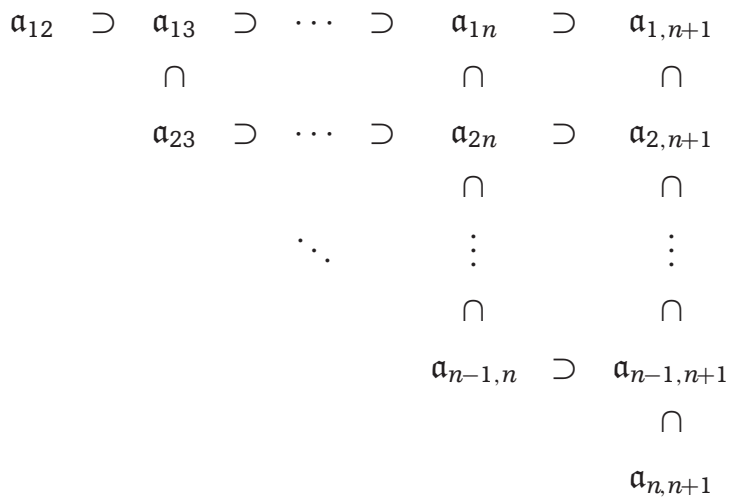

Assume also

$$
\mathfrak{a}_{i j} \cdot \mathfrak{a}_{j k} \subset \mathfrak{a}_{i k}
$$

for all $1 \leq i<j<k \leq n+1$. We define a generalized Auslander algebra as $\mathscr{A}=\oplus_{i, j=1}^{n} \mathscr{A}_{i j}$ with

$$
\mathscr{A}_{i j}= \begin{cases}\mathfrak{a}_{i j} / \mathfrak{a}_{i, n+1} & \text { if } i<j, \\ \mathcal{O}_{S} / \mathfrak{a}_{i, n+1} & \text { if } i \geq j\end{cases}
$$

In other words,

$$
\mathscr{A}:=\left(\begin{array}{ccccc}
\mathcal{O}_{S} & \mathfrak{a}_{12} / \mathfrak{a}_{1, n+1} & \mathfrak{a}_{13} / \mathfrak{a}_{1, n+1} & \cdots & \mathfrak{a}_{1, n} / \mathfrak{a}_{1, n+1} \\
\mathcal{O}_{S} / \mathfrak{a}_{2, n+1} & \mathcal{O}_{S} / \mathfrak{a}_{2, n+1} & \mathfrak{a}_{23} / \mathfrak{a}_{2, n+1} & \cdots & \mathfrak{a}_{2, n} / \mathfrak{a}_{2, n+1} \\
\mathcal{O}_{S} / \mathfrak{a}_{3, n+1} & \mathcal{O}_{S} / \mathfrak{a}_{3, n+1} & \mathcal{O}_{S} / \mathfrak{a}_{3, n+1} & \cdots & \mathfrak{a}_{3, n} / \mathfrak{a}_{3, n+1} \\
\vdots & \vdots & \vdots & \ddots & \vdots \\
\mathcal{O}_{S} / \mathfrak{a}_{n, n+1} & \mathcal{O}_{S} / \mathfrak{a}_{n, n+1} & \mathcal{O}_{S} / \mathfrak{a}_{n, n+1} & \cdots & \mathcal{O}_{S} / \mathfrak{a}_{n, n+1}
\end{array}\right)
$$

The multiplication is induced by the natural embedding

$$
\mathscr{A} \subset \operatorname{End}\left(\mathcal{O}_{S} / \mathfrak{a}_{1, n+1} \oplus \mathcal{O}_{S} / \mathfrak{a}_{2, n+1} \oplus \mathcal{O}_{S} / \mathfrak{a}_{3, n+1} \oplus \cdots \oplus \mathcal{O}_{S} / \mathfrak{a}_{n, n+1}\right)
$$


Example A.15. Let $\mathfrak{r} \subset \mathcal{O}_{S}$ be an ideal such that $\mathfrak{r}^{n}=0$. Then

$$
\mathfrak{a}_{i j}=\mathfrak{r}^{n+1-i}: \mathfrak{r}^{n+1-j}
$$

gives $\mathscr{A}=\operatorname{End}\left(\mathcal{O}_{S} \oplus \mathcal{O}_{S} / \mathfrak{r} \oplus \mathcal{O}_{S} / \mathfrak{r}^{2} \oplus \cdots \oplus \mathcal{O}_{S} / \mathfrak{r}^{n-1}\right)$, the original Auslander algebra.

Example A.16. Let $\mathfrak{r} \subset \mathcal{O}_{S}$ be an ideal such that $\mathfrak{r}^{n}=0$. Then

$$
\mathfrak{a}_{i j}=\mathfrak{r}^{j-i}
$$

gives the algebra (30), the special Auslander algebra.

It turns out that the generalized Auslander algebras enjoy the same properties as the special Auslander algebras considered in Section 5. For example, the argument of Proposition 5.14 and Corollary 5.15 proves the following proposition.

Proposition A.17. There are semiorthogonal decompositions

$$
\begin{aligned}
\mathbf{D}(\mathscr{A}) & =\left\langle\mathbf{D}\left(S_{1}\right), \mathbf{D}\left(S_{2}\right), \ldots, \mathbf{D}\left(S_{n}\right)\right\rangle \\
\mathbf{D}^{b}(\operatorname{coh}(\mathscr{A})) & =\left\langle\mathbf{D}^{b}\left(\operatorname{coh}\left(S_{1}\right)\right), \mathbf{D}^{b}\left(\operatorname{coh}\left(S_{2}\right)\right), \ldots, \mathbf{D}^{b}\left(\operatorname{coh}\left(S_{n}\right)\right)\right\rangle .
\end{aligned}
$$

where $S_{i}$ is the subscheme of $S$ corresponding to the ideal $\mathfrak{a}_{i, i+1}$, so that $\mathcal{O}_{S_{i}}=\mathcal{O}_{S} / \mathfrak{a}_{i, i+1}$.

Remark A.18. Note that the components of the decomposition do not depend on the ideals $\mathfrak{a}_{i, j}$ with $j-i>1$. However, one can check that these ideals govern the gluing functors of the decomposition.

For the special Auslander algebra we have $\mathfrak{a}_{i, i+1}=\mathfrak{r}$ for all $i$, hence $S_{1}=\cdots=S_{n}=$ $S_{0}$, so all the components of the semiorthogonal decomposition coincide. On the other hand, for the original Auslander algebra we have $\mathfrak{a}_{i, i+1}=\mathfrak{r}^{n+1-i}: \mathfrak{r}^{n-i}$, so the components may be different.

Example A.19. Consider $S=\operatorname{Spec} \mathbb{k}[x, y] /\left(x^{2}, x y\right)$. Take $\mathfrak{r}=(x)$ and $n=2$. Then we have $\mathfrak{a}_{1,2}=\operatorname{Ann}(\mathfrak{r})=(x, y), \mathfrak{a}_{1,3}=0, \mathfrak{a}_{2,3}=\mathfrak{r}$, so $S_{1}=\operatorname{Spec} \mathbb{k}, S_{2}=\operatorname{Spec} \mathbb{k}[y]$. In particular, the original Auslander algebra gives a semiorthogonal decomposition with components $\mathbf{D}(\mathbb{k})$ and $\mathbf{D}(\mathbb{k}[y])$, while the special Auslander algebra gives a decomposition with both components equal to $\mathbf{D}(\mathbb{k}[y])$. 
Proposition A.20. In the assumptions of Proposition A.17, assume that the subschemes $S_{1}, \ldots, S_{n} \subset S$ are local regular schemes of dimensions $d_{1}, \ldots, d_{n}$. Then

$$
\operatorname{gldim} \mathscr{A} \leq d_{1}+\cdots+d_{n}+2(n-1), \quad \operatorname{gldim} \mathscr{A}^{\mathrm{op}} \leq d_{1}+\cdots+d_{n}+2(n-1)
$$

Proposition A.21. In the assumptions of Proposition A.17, if all the subschemes $S_{1}, \ldots, S_{n}$ of $S$ are smooth, then

$$
\mathbf{D}(\mathscr{A})^{\mathrm{c}}=\mathbf{D}^{\text {perf }}(\mathscr{A})=\mathbf{D}^{b}(\operatorname{coh}(\mathscr{A})) .
$$

Again, one can prove these propositions by the same arguments as the corresponding statements in Sections 5 and A.6.

Moreover, one can define a DG-category $\mathscr{D}(\mathscr{A})$ in the same way as it is done in Section 5, construct a DG-functor $\rho_{S}: \mathscr{D}(S) \rightarrow \mathscr{D}(\mathscr{A})$. Again, the same arguments prove the following theorem.

Theorem A.22. In the assumptions of Proposition A.17, if all the subschemes $S_{1}, \ldots, S_{n}$ of $S$ are smooth, then the functor $\rho_{S}$ is a DG-resolution of singularities and the derived functor $L \rho_{S}^{*}: \mathbf{D}(S) \rightarrow \mathbf{D}(\mathscr{A})$ is a categorical resolution.

\section{A.8 Generalized morphisms of $\mathscr{A}$-spaces}

In this section, we describe the notion of generalized morphisms of $\mathscr{A}$-spaces. Let $(S, \mathfrak{a})$ be a generalized Auslander space as defined in Section A.7.

Definition A.23. A generalized morphism of $\mathscr{A}$-spaces from $\left(T, \mathfrak{a}^{T}, n_{T}\right)$ to $\left(S, \mathfrak{a}^{S}, n_{S}\right)$ consists of a continuous map $f: T \rightarrow S$ of the underlying topological spaces and a sheaf $\mathcal{P}$ of $f^{-1} \mathscr{A}_{S^{-}} \mathscr{A}_{T}$-bimodules which is projective over $\mathscr{A}_{T}$.

If $\mathbf{f}=(f, \mathcal{P})$ is a morphism of $\mathscr{A}$-spaces, we can define the functors

$$
\begin{gathered}
\mathbf{f}^{*}: \operatorname{Ocoh}\left(\mathscr{A}_{S}\right) \rightarrow \operatorname{Qcoh}\left(\mathscr{A}_{T}\right), \quad M \mapsto f^{-1} M \otimes_{f^{-1} \mathscr{A}_{S}} \mathcal{P}, \\
\mathbf{f}_{*}: \operatorname{Qcoh}\left(\mathscr{A}_{T}\right) \rightarrow \operatorname{Qcoh}\left(\mathscr{A}_{S}\right), \quad N \mapsto f_{*} \mathcal{H o m}_{\mathscr{A}_{T}}(\mathcal{P}, N) .
\end{gathered}
$$

One can easily prove the following lemma.

Lemma A.24. The functor $\mathbf{f}^{*}$ is right exact and the functor $\mathbf{f}_{*}$ is left exact. Moreover, the functor $\mathbf{f}^{*}$ is the left adjoint of the functor $\mathbf{f}_{*}$. 
Assume that $f: T \rightarrow S$ is a morphism of schemes compatible with ideals $\mathfrak{a}_{i j}^{T}$ and $\mathfrak{a}_{i j}^{S}$, that is,

$$
f^{-1}\left(\mathfrak{a}_{i j}^{S}\right) \subset \mathfrak{a}_{i j}^{T}
$$

for all $1 \leq i<j \leq n_{S}+1$ and $n_{S} \leq n_{T}$. Then, the morphism $f$ induces a morphism $f^{-1} \mathscr{A}_{S} \rightarrow$ $\mathscr{A}_{T}$ compatible with the addition and multiplication laws and taking the unit of $\mathscr{A}_{S}$ to the idempotent $\epsilon_{1}+\cdots+\epsilon_{n_{S}}$ of $\mathscr{A}_{T}$. Thus,

$$
\mathcal{P}_{f}:=\left(\epsilon_{1}+\cdots+\epsilon_{n_{S}}\right) \mathscr{A}_{T}
$$

is a $f^{-1}\left(\mathscr{A}_{S}\right)-\mathscr{A}_{T}$-bimodule which is projective over $\mathscr{A}_{T}$. So, the pair $\mathbf{f}:=\left(f, \mathcal{P}_{f}\right)$ defines a generalized morphism of $\mathscr{A}$-spaces, which we will refer to as the morphism of $\mathscr{A}$ spaces induced by the morphism $f$ of schemes. It is easy to see that the induced pullback and pushforward functors on categories of $\mathscr{A}$-modules coincide with those defined in Section 5.

Example A.25. Let $S_{1} \subset S$ be the subscheme corresponding to the ideal $\mathfrak{a}_{1,2} \subset \mathcal{O}_{S}$. Then, we have a generalized morphism from $\left(S_{1}, 0,1\right)$ to $\left(S, \mathfrak{a}^{S}, n_{S}\right)$ given by the embedding of schemes $i: S_{1} \rightarrow S$ and the bimodule $\mathcal{P}_{1}=\mathcal{O}_{S_{1}}$. Then, the corresponding pullback and pushforward functors coincide with the functors $i^{*}$ and $i$ investigated in Section 5.

On the other hand, let $S^{\prime} \subset S$ be the subscheme corresponding to the ideal $\mathfrak{a}_{2, n+1} \subset \mathcal{O}_{S}$ and let $\mathscr{A}^{\prime}$ be the generalized Auslander algebra corresponding to the system of ideals in $\mathcal{O}_{S^{\prime}}=\mathcal{O}_{S} / \mathfrak{a}_{2, n+1}$ obtained from (A.7) by removing the first line. Note that the schemes $S$ and $S^{\prime}$ have the same underlying topological spaces. Let $e: S^{\prime} \rightarrow S$ be the identity morphism of those (note that it does not extend to a morphism of schemes). Further, $\mathcal{P}^{\prime}=\left(1-\epsilon_{1}\right) \mathscr{A}$ is a $\mathscr{A}^{\prime}-\mathscr{A}$-bimodule which is projective over $\mathscr{A}$, hence e $=\left(e, \mathcal{P}^{\prime}\right)$ is a morphism of $\mathscr{A}$-spaces. Then the pullback functor $\mathrm{e}^{*}$ is isomorphic to the functor e, while the pushforward functor $\mathbf{e}_{*}$ is isomorphic to $e^{!}$, defined in (39) and (41), respectively.

It is also easy to see that the induction and the restriction functors considered in Lemma 5.6 are also the pullback and the pushforward for appropriate generalized morphisms of $\mathscr{A}$-spaces.

\section{References}

[1] Alonso, L., A. Jeremias, and J. Lipman. "Local homology and cohomology on schemes." Annales Scientifiques de l'École Normale Supérieure. Quatriéme Série 30, no. 1 (1997): 1-39. 
[2] Auslander, M. "Representation Dimension of Artin Algebras." In Selected works of Maurice Auslander. Part 1, edited by I. Reiten, S. O. Smalø, and Ø. Solberg. Providence, RI: American Mathematical Society, 1999.

[3] Berthelot, P., A. Grothendieck, and L. Illusie. Théorie des réductions et théorème de Riemann-Roch. Lecture Notes in Mathematics 225. Heidelberg: Springer, 1971.

[4] Bierstone, E. and P. Milman. "Canonical desingularization in characteristic zero by blowing up the maximum strata of a local invariant." Inventiones Mathematicae 128, no. 2 (1997): 207-302.

[5] Bierstone, E. and P. Milman. "Functoriality in resolution of singularities." Research Institute for Mathematical Sciences. Publications. 44, no. 2 (2008): 609-39.

[6] Bondal, A. and M. Kapranov. "Representable functors, Serre functors, and reconstructions." Izvestiya Akademii Nauk SSSR. Seriya Matematicheskaya 53, no. 6 (1989): 1183-205, 1337 (Russian); translation in Mathematics of the USSR-Izvestiya 35, no. 3 (1990): 519-41.

[7] Bondal, A. and M. Kapranov. "Framed triangulated categories." Matematicheskii Sbornik 181, no. 5 (1990): 669-83 (Russian); translation in Math. USSR-Sb. 70, no. 1 (1991): 93-107.

[8] Bondal, A. and D. Orlov. "Semiorthogonal decomposition for algebraic varieties." (1995): preprint math.AG/9506012.

[9] Bondal, A. and D. Orlov. "Derived Categories of Coherent Sheaves." Proceedings of the International Congress of Mathematicians, Vol. II (Beijing, 2002), 47-56. Beijing: Higher Ed. Press, 2002.

[10] Drinfeld, V. "DG quotients of DG categories." Journal of Algebra 272, no. 2 (2004): 643-91.

[11] Efimov, A. "Homotopy finiteness of some DG categories from algebraic geometry." (2013): preprint arXiv:1308.0135.

[12] Hartshorne, R. Algebraic Geometry, xvi+496 pp. Graduate Texts in Mathematics 52. New York-Heidelberg: Springer, 1977.

[13] Kashiwara, M. and P. Schapira, Categories and Sheaves. Grundlehren der Mathematischen Wissenschaften [Fundamental Principles of Mathematical Sciences] 332. Berlin: Springer, 2006.

[14] Keller, B. On Differential Graded Categories. International Congress of Mathematicians II, 151-90. Zürich: European Mathematical Society, 2006.

[15] Kuznetsov, A. "Lefschetz decompositions and categorical resolutions of singularities." Selecta Mathematica 13, no. 4 (2008): 661-96.

[16] Lunts, V. “Coherent sheaves on configuration schemes." Journal of Algebra 244, no. 2 (2001): $379-406$.

[17] Lunts, V. "Categorical resolution of singularities." Journal of Algebra 323, no. 10 (2010): 2977-3003.

[18] Lunts, V. "Categorical resolutions, poset schemes and Du Bois singularities." Int. Math. Res. Not. IMRN no. 19 (2012): 4372-4420.

[19] Lunts, V. and D. Orlov. "Uniqueness of enhancement for triangulated categories." Journal of American Mathematical Society 23, no. 3 (2010): 853-908. 
[20] Lunts, V. and O. Schnürer. "Smoothness of equivariant derived categories." Proceedings of the London Mathematical Society, 2013. doi: 10.1112/plms/pdt053

[21] Neeman, A. "The connection between the $K$-theory localisation theorem of Thomason, Trobaugh and Yao, and the smashing subcategories of Bousfield and Ravenel." Annales Scientifiques de l'École Normale Supérieure. Quatriéme Série 25, no. 5 (1992): 547-66.

[22] Neeman, A. "The Grothendieck duality theorem via Bousfield's techniques and Brown representability." Journal of the American Mathematical Society 9, no.1 (1996): 205-36.

[23] Neeman, A. Triangulated Categories. Annals of Mathematics Studies 148. Princeton, NJ: Princeton University Press, 2001.

[24] Spaltenstein, N. "Resolutions of unbounded complexes." Compositio Mathematica 65, no. 2 (1988): 121-54.

[25] Tabuada, G. “Theorie homotopique des DG-categories." (2008): preprint arXiv:0710.4303.

[26] Toën, B. and M. Vaquié. "Moduli of objects in dg-categories." Annales Scientifiques de l'École Normale Supérieure 40, no. 3 (2007): 387-444.

[27] Toën, B. "The homotopy theory of $d g$-categories and derived Morita theory." Inventiones Mathematicae 167, no. 3 (2007): 615-67.

[28] Van den Bergh, M. Non-Commutative Crepant Resolutions. The Legacy of Niels Hendrik Abel, 749-70. Berlin: Springer, 2004. 